\title{
The triphasic mechanics of the intervertebral disc : a theoretical, numerical and experimental analysis
}

Citation for published version (APA):

Snijders, J. M. A. (1994). The triphasic mechanics of the intervertebral disc : a theoretical, numerical and experimental analysis. [Doctoral Thesis, Maastricht University]. Datawyse / Universitaire Pers Maastricht. https://doi.org/10.26481/dis.19940128js

Document status and date:

Published: 01/01/1994

DOI:

10.26481/dis.19940128js

Document Version:

Publisher's PDF, also known as Version of record

\section{Please check the document version of this publication:}

- A submitted manuscript is the version of the article upon submission and before peer-review. There can be important differences between the submitted version and the official published version of record.

People interested in the research are advised to contact the author for the final version of the publication, or visit the DOI to the publisher's website.

- The final author version and the galley proof are versions of the publication after peer review.

- The final published version features the final layout of the paper including the volume, issue and page numbers.

Link to publication

\footnotetext{
General rights rights.

- You may freely distribute the URL identifying the publication in the public portal. please follow below link for the End User Agreement:

www.umlib.nl/taverne-license

Take down policy

If you believe that this document breaches copyright please contact us at:

repository@maastrichtuniversity.nl

providing details and we will investigate your claim.
}

Copyright and moral rights for the publications made accessible in the public portal are retained by the authors and/or other copyright owners and it is a condition of accessing publications that users recognise and abide by the legal requirements associated with these

- Users may download and print one copy of any publication from the public portal for the purpose of private study or research.

- You may not further distribute the material or use it for any profit-making activity or commercial gain

If the publication is distributed under the terms of Article $25 \mathrm{fa}$ of the Dutch Copyright Act, indicated by the "Taverne" license above, 


\section{The triphasic mechanics of the}

\section{intervertebral disc}

-a theoretical, numerical and experimental analysis- 
CIP-GEGEVENS KONINKLIJKE BIBLIOTHEEK, DEN HAAG

Snijders, Johannes Maria Abraham

The triphasic mechanics of the interwertebral disc: a theoretical, numerical and experimental analysis /

Johannes Maria Abraham Snijders. - Maastricht :

Universitaire Pers Maastricht. - Met lit, opg. - Met samenvatting in het Nederlands.

ISBN 90-5278-125-7

Trefw.: biomechanica. 


\title{
The triphasic mechanics of the intervertebral disc
}

-a theoretical, numerical and experimental analysis-

\author{
PROEFSCHRIFT
}

ter verkrijging van de graad van doctor aan de Rijksuniversiteit Limburg te Maastricht, op gezag van de Rector Magnificus, Prof. Dr. H. Philipsen, volgens het besluit van het College van Dekanen, in het openbaar te verdedigen op vrijdag 28 januari 1994 om 16.00 uur

door

Johannes Maria Abraham Snijders

geboren te Heerlen op 20 mei 1959 
Promotor

Co-promotor

Beoordelingscommissie
Prof. Dr. Ir. J.D. Janssen

Dr. Ir. J.M.R.I. Huyghe

Prof. Dr. G.J. van der Vusse (voorzitter)

Prof. Dr. Ir. R. de Borst (TU Delft)

Prof. Dr. A. Huson

Prof. Dr. Y. Lanir (Israel Institute of Technology)

Prof. Dr. Ir. Drs. O.J. Vrieze 
$A^{\alpha} \quad$ specific Helmboltz free energy

B semi positive definite tensor

$c^{\alpha} \quad$ concentration per unit fluid volume

$\mathrm{C}^{\infty} \quad$ concentration per unit mixture (tissue) volume

D diffusion tensor

$\mathrm{e}^{\mathrm{a}} \quad$ mass production due to other constituents

E Green Lagrange strain tensor

$\mathrm{f}^{\text {tu }} \quad$ activity coefficient

E deformation tensor

$\mathrm{g}^{\text {a }} \quad$ heat supply due to other constituents

$\mathrm{j}^{\alpha}$ heat flux

$\underline{K}$ permeability tensor

$\mathrm{m}^{\alpha} \quad$ number of moles

$\mathrm{M}^{\infty} \quad$ molar weight

$\mathrm{n}^{\alpha} \quad$ volume fraction

$\overrightarrow{\mathrm{n}} \quad$ unit normal vector

$\vec{q}^{\alpha} \quad$ body force per unit mass

$\mathrm{p} \quad$ fluid pressure

$P_{\text {app }} \quad$ applied pressure

$P_{c} \quad$ collagen network stress

$\mathrm{P}_{\mathrm{s}} \quad$ swelling pressure

$r^{s} \quad$ external specific heat supply

$\mathrm{R} \quad$ universal gas constant

S second Piola Kirchhoff stress tensor

$\mathrm{t}$ time

$\mathrm{T} \quad$ absolute temperature

$\mathrm{x} \quad$ mole fraction

$\overrightarrow{\mathrm{u}} \quad$ displacement vector

u fluid velocity relative to solid velocity

$\vec{U} \quad$ solid velocity relative to ion velocity

w fluid velocity relative to ion velocity

V velocity vector

$\overrightarrow{\mathrm{x}} \quad$ position vector

$\epsilon^{e} \quad$ specific internal energy

$\gamma^{\alpha} \quad$ entropy flux

$\lambda$ axial siretch

$\kappa, \lambda \quad$ Lagrange multipliers

$\rho^{\alpha} \quad$ mass density per unit mixture (tissue) volume

$\mathrm{e}^{\alpha} \quad$ mass density per unit fluid volume

$\Gamma^{\mathrm{c}} \quad$ intrinsic mass density

$\eta^{\alpha} \quad$ specific entropy

$0^{\alpha} \quad$ Cauchy stress tensor

$\mu^{\alpha} \quad$ chemical potential

क osmotic retention

$\pi \quad$ pressure

$\bar{\pi}^{c} \quad$ momemtum supplied by the other constituents 


$\begin{array}{ll}\text { app } & \text { applied } \\ \mathrm{c} & \text { collagen } \\ \text { eff } & \text { effective } \\ s & \text { swelling } \\ u & \text { solid relative to fluid } \\ & \text { ion relative to solid } \\ \text { w } & \text { ion rejative to fluid } \\ 0 & \text { reference state }\end{array}$

\section{Superscripts}

$\begin{array}{ll}c p & \text { proteoglycan } \\ f & \text { fluid } \\ i & \text { ions } \\ \$ & \text { solid } \\ + & \text { cation } \\ - & \text { anion } \\ \alpha & \text { arbitrary constituent }\end{array}$

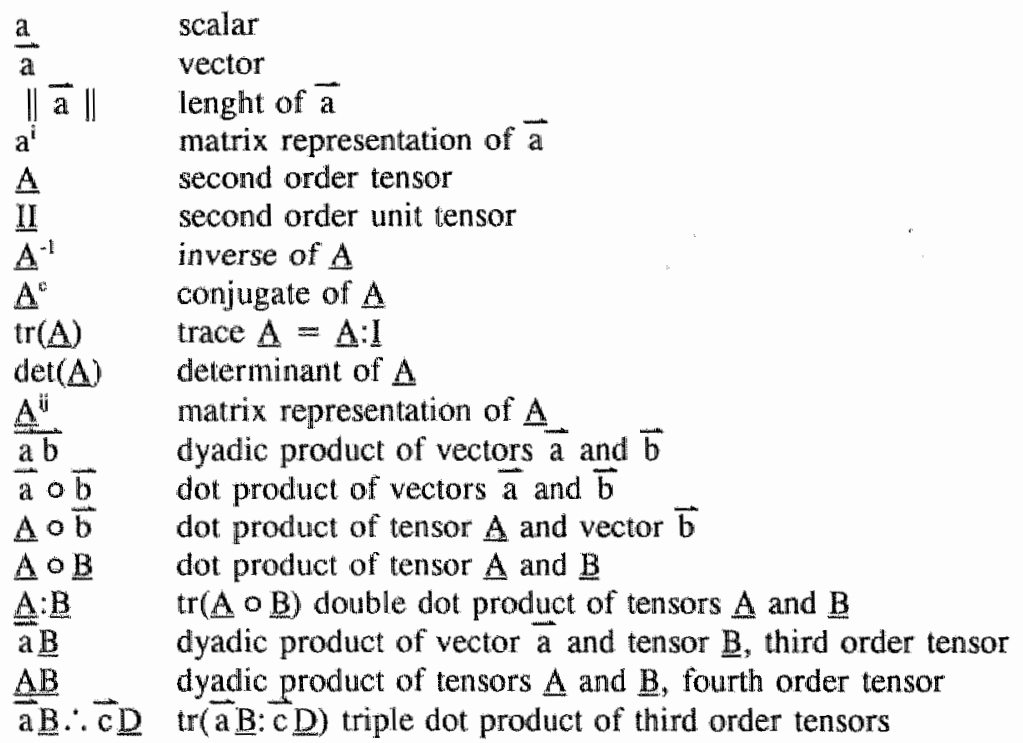


SYMBOLS, SUBSCRIPTS, SUPERSCRIPTS AND NOTATION

1 INTRODUCTION 11

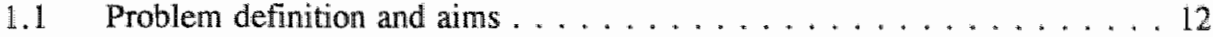

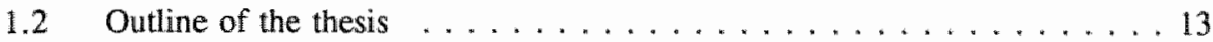

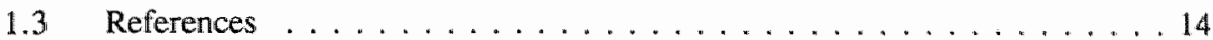

2 THE MOTION SEGMENT 15

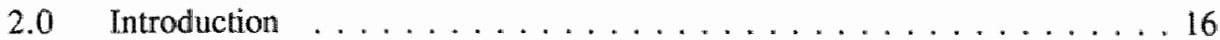

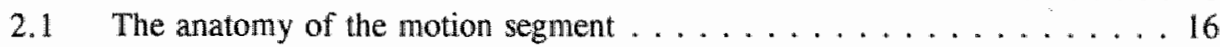

2.2 Biochemical composition . . . . . . . . . . . . . . . . 17

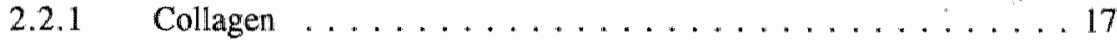

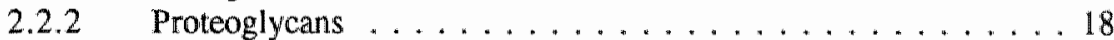

2.3 Tissue composition and mechanical function ................ 19

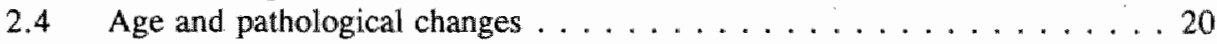

2.5 Existing models for swelling soft tissues $\ldots \ldots \ldots \ldots \ldots \ldots \ldots \ldots$

2.5 .1 Donnan equilibria . . . . . . . . . . . . . . . 21

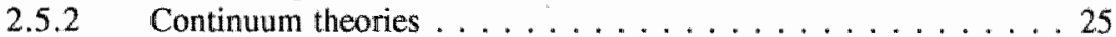

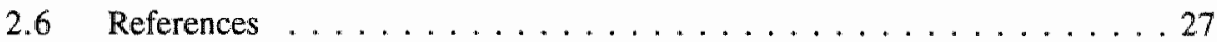

3. TRIPHASIC THEORY 31

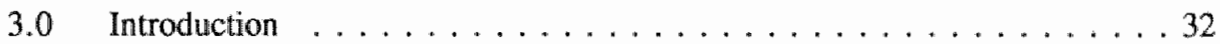

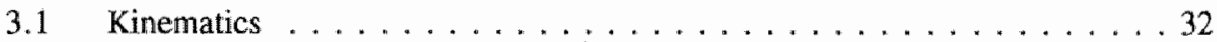

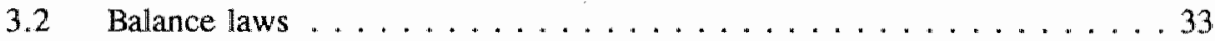

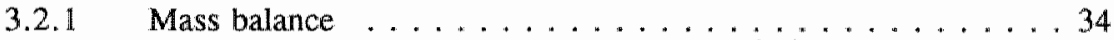

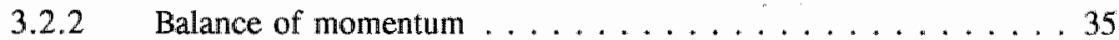

3.2 .3 Balance of moment of momentum . . . . . ....... 35

3.2.4 Balance of energy or

3.2.5 Entropy inequality or

first axiom of thermodynamics .......... 36

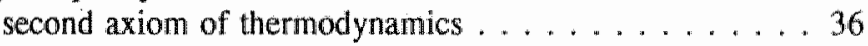

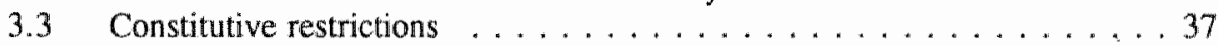

3.3.1 Identification of Lagrange multipliers . . . . . . . . . . . 40

3.3.2 The momentum interactions . . . . . . . . . . . 42

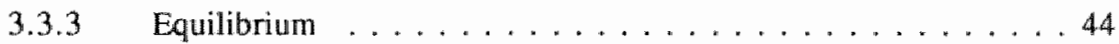

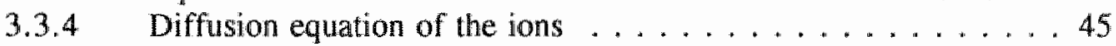

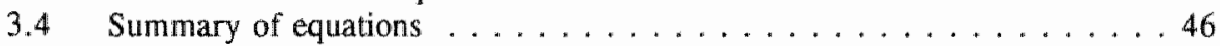

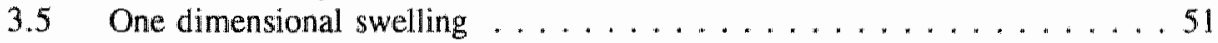

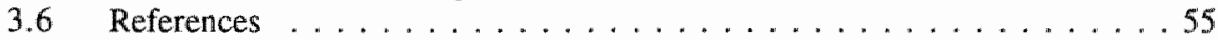


4 NUMERICAL SOLUTION METHOD

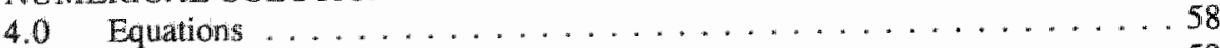

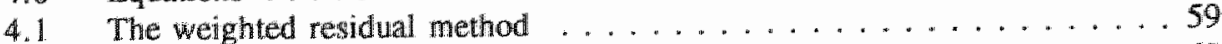

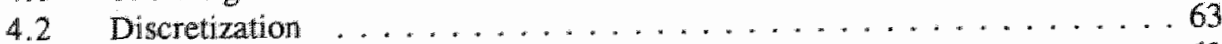

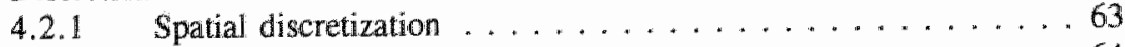

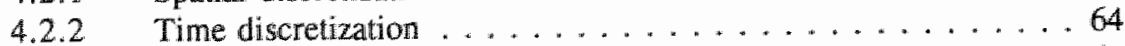

4.2 .3 Discretization of the weighted residual equations ......... 64

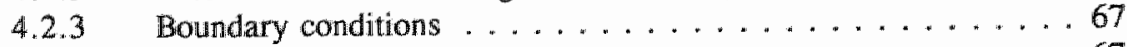

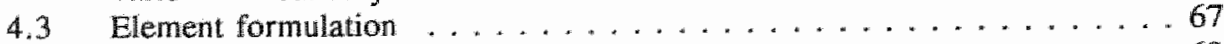

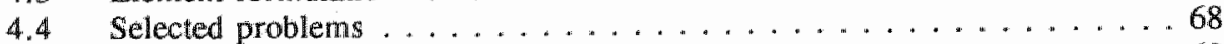

4.4.1 Dispersion in laminar flow: Taylor dispersion ......... 68

4.4.2 Finite inflation of a single-phase, thick,

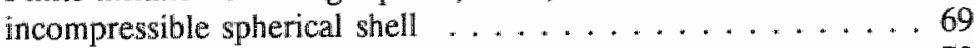

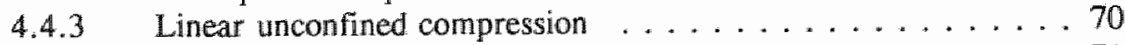

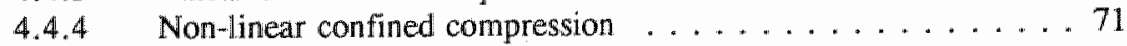

4.5 Compression of a schematic motion segment . . . . . . . . . . 74

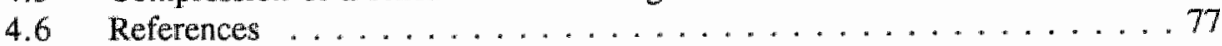

5 BIPHASIC VERSUS TRIPHASIC CONFINED COMPRESSION 79

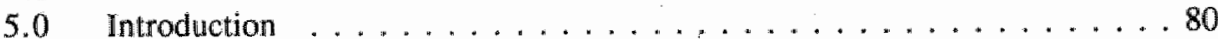

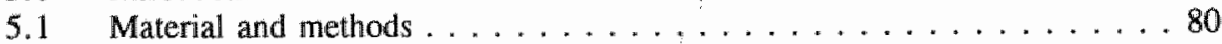

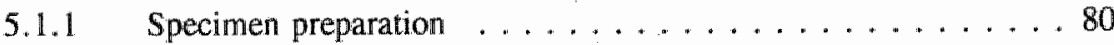

5.1 .2 Experimental set-up ..................... 84

5.1 .3 Experimental protocol ................... 85

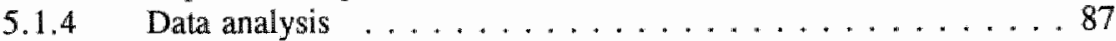

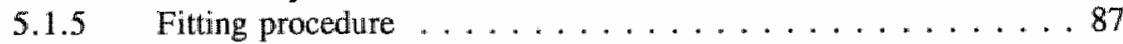

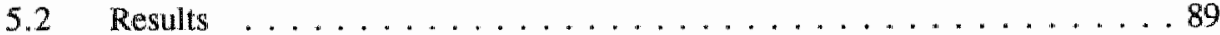

5.2 .1 Histology . . . . . . . . . . . . . . . . . . . . . . . . . . . . . . . . . . . . . . . . .

5.2 .2 Displacement . . . . . . . . . . . . . . . 89

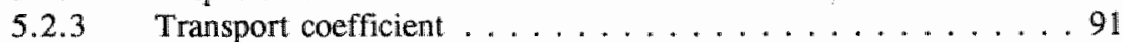

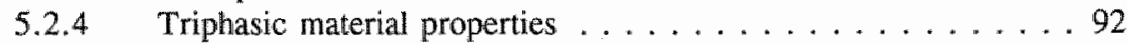

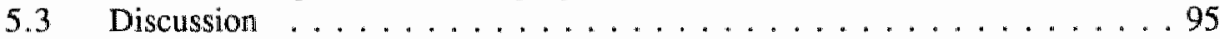

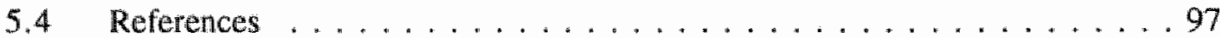

6 AXIAL COMPRESSION OF A MOTION SEGMENT 99

6.0 Introduction . . . . . . . . . . . . . . . . . . . . . 100

6.1 Materials and methods ........................ 100

6.1 .1 Theory ............................. 100

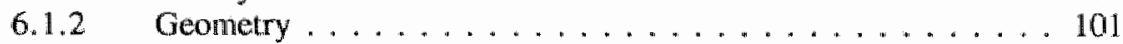

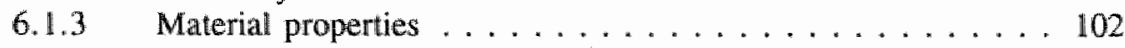

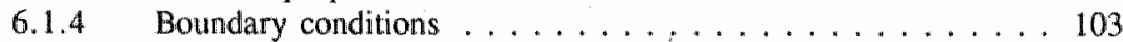

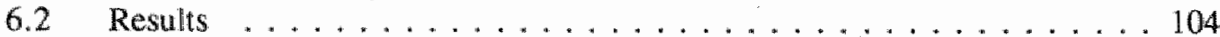

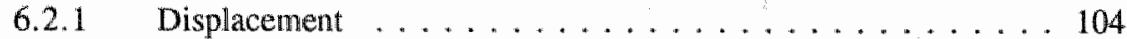

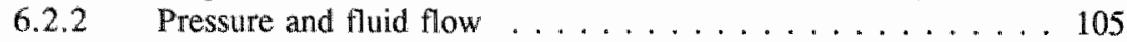

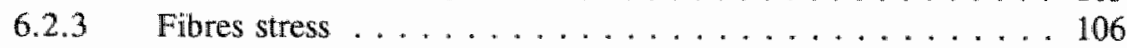

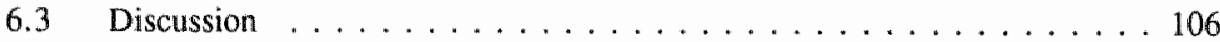

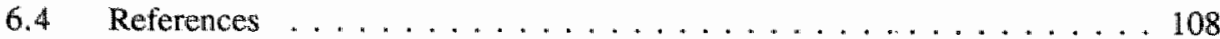


7 SUMMARY, CONCLUSIONS AND RECOMMENDATIONS

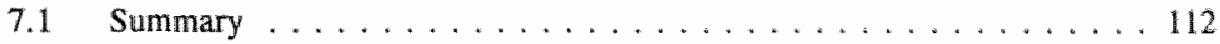

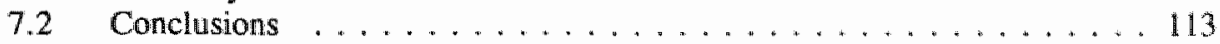

7.2.1 Conclusions concerning the presented theory ......... 113

7.2.2 Conclusions conceming the experimental method and data ... 114

7.2.3 Conclusions concerning the numerical simulations . . . . . 115

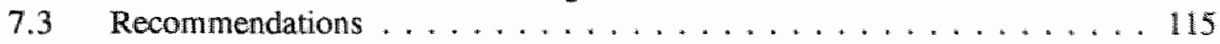

7.3.1 Recommendations concerning the presented theory ..... 15

7.3.2 Recommendations concerning the experimental method ..... 115

7.3.3. Recommendations concerning the numerical simulations ... 115

7.4 References

SAMENVATTING $\quad 116$

$\begin{array}{ll}\text { CURRICULUM VITAE } & 118\end{array}$ 



\section{INTRODUCTION}




\subsection{Problem definition and aims}

In the industrial world low back pain is one of the important causes of absence and incapacily for work. Vermeer (1983) estimates the costs related to low back pain for 1981 in the Netherlands on 1 billion US dollars. Besides the financial loss there is of course the personal loss of quality of life.

Low back pain is split into two categories: acute (pain period less than 3 months) and chronic pain. In 90\% the acute pain is caused by mechanical damage (Nachemson, 1982). The slipped disc is one of the best examples. Through ruptures in the outer regions of the intervertebral disc tissue from the interior is expelled, Ultimately this tissue irritates the nerve root in the spinal canal. If the chronic pain continues over 1 year the nature is for $80 \%$ psychosocial. Epidemiological investigations relate the incidence of low back pain to the mechanical load during work and daily activity (Chaffin, 1982).

The basic components of intervertebral disc tissue are: the groundsubstance, the collagen and elastin fibres and the interstitial fluid. Each of these components contribute to the load bearing capacity. (1) Due to the incompressibility the groundsubstance and the fluid are able to withstand a pressure load. (2) The fibres are dedicated to counterbalance tension. (3) The ionized groundsubstance is entangled in the fibre network. With respect to its environment, not including the same substance, osmotic effects are important. Intervertebral disc tissue behaves as a semi-permeable continuum. Osmotic effects result in a swelling tendency, protecting the groundsubstance in a compressive situation.

The incidence of low back pain is maximum between the third and fifth decade (Snook, 1982). Mostly during this time period degeneration of the intervertebral disc takes place. This may indicate that the degeneration of intervertebral discs plays an important role. Whether the changes, which are associated with degeneration, are the result of a natural process related to aging is not clear yet. Degeneration manifests as biochemical and structural changes of the disc, the cartilaginous endplate and the vertebral body. These changes result in a decrease of the osmotic protection of the groundsubstance. Generally the degenerated disc is less able to withstand the task of load transmission. At lower load levels micro trauma occurs. Aging also decreases the ability of repair mechanisms. Micro trauma grows to ruptures and fissures. It is evident that mechanical loading combined with degeneration or aging plays a key role in low back pain development.

Mechanical analysis of the spinal column might give insight in the load transmission. Generally this mechanical modelling is done on two levels. Macro models study the whole spine without modelling anatomic structures in detail. Macro models render the global loading conditions on the different macroscopic components of the spine. Micro models study the repeating unit: the motion segment. A motion segment consists of at least two adjacent vertebral bodies with the arches, the facet joints and the intervertebral disc. Several ligaments and muscles attach to the motion segment. Anatomical details such as geometry, composition and structure of the tissue are included. Micro models render valuable information about the load transmission through the different structures of the 
motion segment. Detailed information about the role of the different components is obtained. For instance the relative contribution of the osmotic pressure versus the contribution of the elastic deformation to the stiffness of the interwertebral disc. The reliability of the results depends on the accuracy of the knowledge of the material behaviour of the different tissue types involved. This information is obtained by mechanical testing of representative samples. Soft biological tissues are highly bydrated. In order to prevent dehydration the tests are performed in an aqueous solution or at $100 \%$ relative humidity. Dependent on the ionic strength of the solution soft tissues swell. In deionized water nucleus samples may increase their volume up to $200 \%$ of the original volume. Even in physiological saline substantial swelling occurs. The swelling alters the geometry and composition of the tissue sample. Mechanical loading is thus not the only mechanism for deformation, chemical loading also results in deformation of the tissue. Mechanical characterisation is always done relative to a reference state. In soft biological research not only the deformation behaviour but also the reference state depends on the ionic surroundings. Commonly one assumes the existence of a stress-free state, and one chooses this state as the reference state. It is obvious that the osmotic prestressing of the intervertebral disc does not allow to equate the unloaded state to stress-free state. The objective of this work is:

-the derivation of a theoretical framework based on the theory of mixtures which takes into account the biochemical and structural composition of the tissue:

- finite deformation of the groundsubstance and fibre network

relative fluid flow

- osmosis

- diffusion of small ions into a charged matrix.

-the finite element modelling of intervertebral disc tissue with special reference to the osmotic prestressing and finite deformations.

-the verification of the model using one-dimensional confined compression and swelling experiments.

-the application of the model to axial loading of a motion segment using an axi-symmetric geometry.

\subsection{Outline of the thesis}

In chapter 2 a functional description of the motion segment is given. Starting with the basic anatomy detailed information about the biochemical and structural composition is given. Age changes or degeneration and pathological changes related to mechanical loading are also discussed. Existing models for swelling soft tissues are summarized.

In chapter 3 a mechanical model for intervertebral disc tissue including "osmotic" effects, ion diffusion and large matrix deformation combined with relative fluid low is developed. In order to describe this complex behaviour the theory of mixtures is used. Starting with kinematic relationships and general balance laws constitutive restrictions 
based on the entropy principle are derived. The consequences of the presented triphasic theory are discussed by means of one-dimensional swelling.

The equations describing the mechanical behaviour of intervertebral disc tissue are three coupled partial differential equations in which geometric and physical non-linearities occur. To solve them for an arbitrary geometry and arbitrary boundary conditions the Finite Element Method is used. In chapter 4 the differential equations are rewritten in an integral form suitable for numerical approximations. Some problems are solved to test the numerical solution method.

In order to test whether the proposed model is realistic one dimensional confined compression experiments on canine samples are performed (chapter 5). The samples are taken from the canine anulus fibrosus. The loading protocol consists of a combination of mechanical and chemical loading.

In chapter 6 the load transmission through a human motion segment is analyzed.

The thesis concludes with a general discussion on the validity and possible applications of the proposed model (chapter 7).

\subsection{References}

Chaffin, D.B. Occupational biomechanics of low back injury. in: Symposium on idiopathic low back pain. (eds. White, A.A, Gordon, S.L.), The C.V. Mosby Company, St. Louis, 323-330, 1982

Nachemson, A.L. The matural cotrse of low back pain. in: Symposium on idiopathic low back pain. (eds. White, A.A, Gordon, S.L.), The C.V. Mosby Company, St. Louis, 46m51, 1982

Snook, S.H. Low back pain in industry. in: Symposium on idiopathic low back pain. (eds. White, A.A, Gordon, S.L.), The C.W. Mosby Company, St. Louis, 23-38, 1982

Vermear, I. Klinische betekenis van degeneratieve afwijkingen van de lumbale wervelkolom. Verzelkeringskundige aspecten bij de beoordeling van arbeidsongeschiktheid. Ned. Tijdschr. Geneesk., 12:1383-138, 1983 
The mechanical response of a motion segment is determined by its anatomical (section 2.1) and structural composition (section 2.3). Not only the gross morphology but also the biochemical composition and interactions (section 2.2) are important. Due to aging the composition characteristics change quite dramatically during lifetime (section 2.4). Existing models for swelling behaviour are outlined in section 2.5 . 


\subsection{Introduction}

In order to study the mechanical response of a motion segment, detailled information is needed about its structural and biochemical characteristics. In this chapter the relevant properties will be discussed. A large variation of properties occurs due to biological variation and spinal level. Moreover the properties change during life. However there are fundamental physical interactions underlying the mechanical behaviour.

Most of the general information can be found in textbooks. For the gross anatomy Williams et al. (1989) is used. Specific information can be obtained from Bogduk and Twomey (1987) and White and Panjabi (1978). For the biochemical composition the review articles of Grodzinsky (1983) and Comper and Laurent (1978) are used. The source of detailed information is referenced in the text. All data are obtained from the average healthy human lower lumbar disc.

\subsection{The anatomy of the motion segment}

The human lumbar spine consists of 5 lumbar vertebrae. The repeating unit, formed by two adjacent vertebral bodies, the intervertebral disc (IVD), the ligaments and the posterior arc with its facet joints, is called the motion segment. In the transwerse plane the intervertebral disc is kidney shaped, except the L.5-S1 disc which is oval. In the sagittal plane the shape is wedged. The IVD consists of three regions: the central nucleus pulposus (NP), the anulus fibrosus (AF) and the vertebral endplates. The AF surrounds the NP peripherally. The endplates cover both the NP and the AF at the top and the bottom. Above the NP and approximately one third of the AF the endplate is cartilaginous (CEP). Above the rest of the AF the endplate is bony (BEP). The BEP is a continuation of the cortical shell of the vertebral body. The boundary between the NP and the AF is not sharply marked. During aging the boundary becomes even less marked.

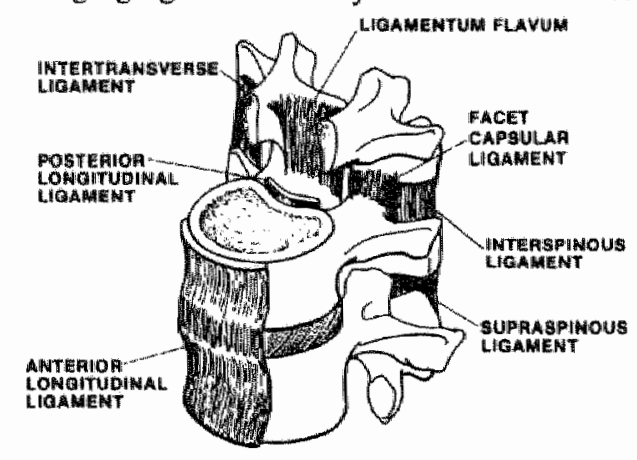

figure 2.1 The motion segment. In order to show all the ligaments a third posterior arc is added (White and Panjabi, 1978). 
The nucleus pulposus forms 25 to $50 \%$ of the sagittal cross area. The NP consists of a few cells and irregularly arranged collagen fibres embedded in a ground substance of hydrated proteoglycans.

In the anulus fibrosus the collagen fibres are embedded in the same ground substance as the NP. The fibres are arranged in 15 to 25 concentric sheets or lamellae around the NP. In the circumferential direction the lamellae may terminate, join or split into wo layers (Marchand and Ahmed, 1990). Within a sheet the fibres lie parallel. Apart from the joining and splitting zones, no fibres run from one sheet to another. Globally the fibre direction is 25 to 30 degrees with the transverse plane, but locally the angle can vary over a wide range (Marchand and Ahmed, 1990). The average angle is 30 degrees (Inove, 1981). The direction of inclination with the transwerse plane alters from left to right with each sheet. The lamellae increase in thickness towards the outer part of the AF.

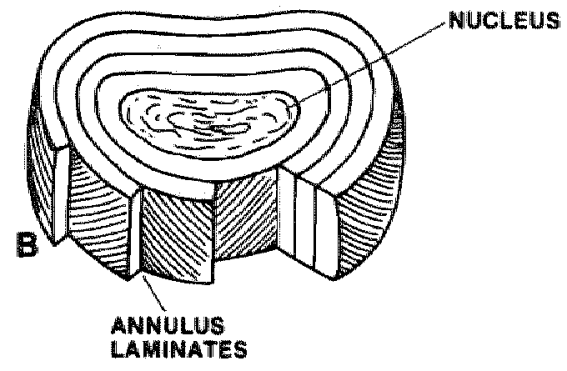

figure 2.2 Schematic view of the intervertebral disc. A part of the anulus fibrosus is removed in order to show the laminate structure (White and Panjabi, 1978)

The CEP is composed of both hyaline cartilage and fibrocartilage. The collagen fibres of the inner AF continue in the CEP: the NP is spherically enveloped by fibres. The fibres in the CEP are densely packed and horizontally aligned. The fibres are not anchored in the sub-chondral bone. The CEP is only weakly attached to the bone (Roberts et al., 1989). Blood vessels and marrow abut directly onto the CEP. Both make the diffusion of nutrients possible into the CEP and the IVD. The collagen fibres of the outer AF are anchored straight in the BEP.

In subsequent chapters the role of IVD tissue is emphasised. Detailed information about the ligaments and the face joints is omitted.

\subsection{Biochemical composition}

\subsubsection{Collagen}

Collagen is a protein built of long polypeptide chains of amino acids. The repeating. monomer is called tropocollagen. This macromolecule consists of three polypeptide chains folded by hydrogen bonds to a triple helical configuration. The total molecular weight is about 300.000 . The rod-like molecule is approximately $300 \mathrm{~nm}$ long and $1.4 \mathrm{~nm}$ in diameter. Five collagen molecules aggregatte to a micro fibre. The latter aggregates to the actual collagen fibres. Within the fibres the tropocollagen are held together by covalent 
bonds. Based on the amino acid sequence 10 types of collagen are distinguished. In the intervertebral disc type I and type II are present. Type I collagen forms thick fibres which have relatively few interactions with the proteoglycans. This type of fibres is found in tissue predominantly subjected to a tensile loading. Type II collagen forms thin fibres and has relatively many interactions with the ground substance. It is predominately found in cissues under pressure. In the IVD type II is found only in the NP. Type I is found in the outer regions of the $\mathrm{NP}$ and $\mathrm{AF}$. In an aqueous solution collagen is a poly electrolyte with positively and negatively charged groups. However at physiological pH these groups are almost self-compensated by intra- and intermolecular electrostatic linkages. The resulting charge densily is slightly positive.

\subsubsection{Proteoglycans}

Proteoglycans (PG) are very large molecules consisting of many glycoaminoglycans (GAG) linked to proteins. The GAG are linear polymeric chains of disaccharide units. The disaccharide units found in the IVD are: chondroitin 4- and 6-sulphate (CS), keratan sulphate (KS) and hyaluronic acid (HA). The GAG chains are bound to a protein core to form a proteoglycan molecule. The molecular weight is approximately $10^{6}$, the length 300 to $400 \mathrm{~nm}$ and the diameter $40 \mathrm{~nm}$. PG chains are linked by a link protein to a long chain of hyaluronic acid to form $\mathrm{PG}$ aggregates. The binding region is rich in the shorter $\mathbb{K S}$, while in the rest the longer $\mathrm{CS}$ is dominant. The PG aggregates entangle and form three dimensional molecular networks. In an aqueous solution at physiological $\mathrm{pH}$ the $\mathrm{PG}$ are ionized. The most important charged groups are the carboxyl group $\left(\mathrm{COO}^{-}\right)$and the sulphate group $\left(\mathrm{SO}_{3}^{-}\right)$of the disaccharide repeat units. On CS both carboxyl and sulphate groups occur, on KS only the latter. Every 0.5 to $1.0 \mathrm{~nm}$ a dissociated group is present, resulting in a very high charge density. Due to the ionization the $P G$ mollecules are capable of attracting and retaining water. The ratio KS/CS for IVD tissue is generally smaller than one (Urban et al., 1979). However the values are scattered. The collagen fibres and the PG aggregates are bound by electrostatic forces. IVD tissue may be viewed upon as a charged fibre reinforced polymer network.
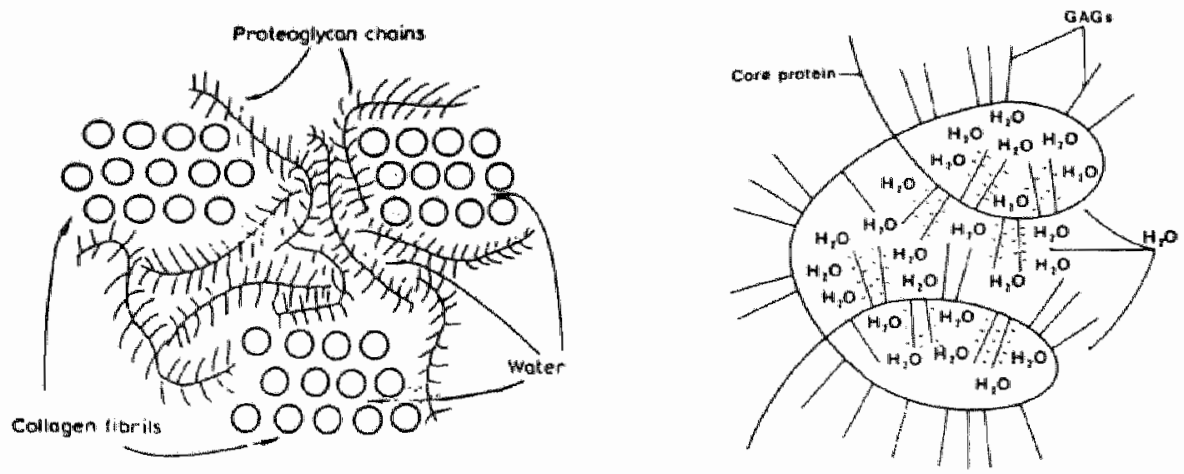

figure 2.3 Schematic view of the tissue composition (left, Urban and McMullin, 1985). Schematic view of the proteoglycan aggregates (right, Bogduk and Twomey, 1987) 


\subsection{Tissue composition and mechanical function}

About 80 to $85 \%$ of the wet weight ( 4 to $6 \mathrm{~g} \mathrm{H} \mathrm{H}_{2} \mathrm{O} / \mathrm{g}$ dry weight) of nucleus tissue is water. The total amount of water is divided over two compartments: the intra and extra. fibrillar water. The intra-fibrillar water is found between the collagen fibres and is invisible for the $\mathbb{P G}$ chains. Urban and McMullin (1985, 1988) estimate this amount as $1.33 \mathrm{~g} \mathrm{H}_{2} \mathrm{O} / \mathrm{g}$ collagen. The extra-fibrillar water is the total amount water within the domain of the $\mathrm{PG}$. The proteoglycans contribute for 50 to $65 \%$ of the dry weight of the nucleus. The resulting FCD is $0.3 \mathrm{meq} / \mathrm{g}$ wet tissue. The collagen contents is $30 \%$ of the dry weight. The composition is rather homogeneous in the nucleus domain.

Biochemically the anulus fibrosus consists of the same components. However the relative concentrations are different. The water content is 60 to $70 \%$ of the total volume (1.5 to $2.5 \mathrm{~g} \mathrm{H}_{2} \mathrm{O} / \mathrm{g}$ dry weight). Collagen contributes for 50 to $60 \%$ to the dry weight, the $\mathrm{PG}$ chains for $20 \%$. The resulting $\mathrm{FCD}$ is $0.2 \mathrm{meq} / \mathrm{g}$ wet tissue. The values are averages. The water content, the $\mathrm{PG}$ concentration and the FCD increase linearly from the outside to the inside (Urban and Maroudas, 1979 and Johnstone et al., 1992). The collagen contents shows the opposite tendency.

The composition of the cartilaginous endplate differs from the ones of the nucleus and anulus. The water content is even lower as in the anulus: $55 \%$ of the tissues wet weight. The collagen contents is even higher than in the anulus: 75 to $80 \%$ of the dry weight. The PG contributes for 10 to $15 \%$ to the dry weight of the tissue. The radial PG and water profiles show the same tendency as the distribution in the nucleus and the anulus. Based on the amount of extra-fibrillar water the FCD of the cartilaginous endplate almost equals the value of the nucleus. The FCD of the anulus is half the nucleus value (Roberts et al., 1989). Note in table 2.1 the FCD is based on the tissues wet weight.

\begin{tabular}{|l|l|l|l|l|}
\hline tissue type & water & collagen & proteoglycan & $\begin{array}{l}\text { fixed charge } \\
\text { density }\end{array}$ \\
\hline nucleus & $80-85$ & 30 & $50-60$ & 0.3 \\
\hline anulus & $60-70$ & $50-60$ & 20 & 0.2 \\
\hline $\begin{array}{l}\text { cartillaginous } \\
\text { endplate }\end{array}$ & 55 & $75-80$ & $10-15$ & 0.1 \\
\hline & $\begin{array}{l}\% \text { wet } \\
\text { weight }\end{array}$ & $\begin{array}{l}\text { \% dry } \\
\text { weight }\end{array}$ & \%dry weight & $\begin{array}{l}\text { meq } / \mathrm{g} \text { wet } \\
\text { tissue }\end{array}$ \\
\hline
\end{tabular}

table 2.1 Global composition of the different tissue types of the intervertebral disc.

In subsequent chapters the detailed mechanical function of the different compartments of the disc is discussed from the point of view of the mixture theory. The mechanics used in the following paragraph is of a rather simple nature. However it is illustrative for the relation between the composition and the mechanical function of the different tissues in the IVD. 
Consider a vertical load transmitted from one vertebral body to the next one. Briefly after the load is applied the IVD behaves like an incompressible "solid". The load is borne by the hydrodynamic fluid pressure. If the load is sustained the situation alters. The pressure first decreases at the boundaries: the CEP and the outer anulus. A pressure gradient exists from the interior to the boundaries. Hence, fluid will be expelled from the IVD to the vertebral body and the outer anulus. The fluid flow is hindered by the water retaining capacity of the ionized proteoglycans. The amount of water attraction is positively correlated to the $\mathrm{PG}$ concentration. When the water attracting force of the proteoglycans and the water expelling force of the pressure gradient are in balance the fluid flow stops. The fluid pressure in the nucleus remains higher than at the outer boundaries. Sometimes the nucleus is even viewed upon as an incompressible fluid ball. The higher pressure in the nucleus strains the anulus radially and circumferentially. The CEP is squeezed between the vertebral body and the nucleus.

In the nucleus the collagen fibres intersperse through the PG matrix. Their primary task is to form a web in which the PG chains are immobilized. The contribution to the intrinsic stiffness may be less important (Urban and McMullin, 1985). In both the CEP and the anulus the collagen fibres are aligned increasing the intrinsic stiffness in the fibre direction. In the anulus the PG chains are found between both the collagen fibres and the lamellae. The ground substance prevents the collagen fibres from buckling or fraying. The intrinsic stiffness of the anulus contributes also to the vertical load transmission.

\subsection{Age and pathological changes}

From the second decade on major biochemical and structural changes occur, especially in the nucleus. With age the concentration of proteoglycans decreases in the nucleus. At the age of 60 it is reduced to half its initial value. The biochemical composition of the proteoglycans changes too: the concentration of chondroitin sulphate (CS) decreases. The concentration of collagen increases, accompanied by an increase of collagen-proteoglycan binding. Due to the electrostatic nature of this binding and the decrease of CS, the FCD decreases vastly. The diameter of the collagen fibres increases. The concentration of type II fibres increases relatively to the type I fibres. In the anulus fibrosus the concentration and the diameter of the collagen decrease. As a result the distinction between the nucleus and the anulus vanishes. The increase of collagen and collagen-proteoglycan interactions makes the IVD stiffer. The decrease of FCD results in a decrease of water binding capacity and a decrease of water content. Hence the nucleus becomes compressible and loses 'osmotic stiffness". The mechanical response and the stress situation of the different tissues alters, e.g. from compression to tension. The contribution to the load transmission of the anulus increases relatively to the nucleus: cracks and cavities may develop. Over years fissures are developed. Whether the biochemical and structural changes are an aging effect or the response to the change in the internal stress distribution is uncertain. The changes could be a metabolic response to the changed stress distribution.

The vertical trabeculae of the vertebral body are slowly absorbed. However the ones that remain are thickened. The horizontal trabeculae demise. The lateral support on the vertical trabeculae declines: the risk of buckling increases. This effect is most marked in 
the central region of the vertebral body. The load bearing capacity of this region decreases and micro fractures occur. Hence, the load bearing capacity of the cancellous part decreases. A greater part of the load is borne by the cortical shell. With age the strength of the cartilaginous vertebral endplate decreases. Lacking support from the underlying bone the endplates run the risk of micro fractures.

It is hypothesized (Bogduk and Twomey, 1987) that through the fractures in the CEP nucleus material may intrude the vertebral body. As both the vertebral body and the CEP are vascular, nucleus tissue, which in healthy conditions has no blood supply, is exposed to the blood circulation. Hence, the proteins of nucleus tissue are recognised as foreign. An auto-immune response is evoked, resulting in a degeneration of the tissue.

In general the origin of low back pain is hard to trace. A well known cause is disc herniation. "Through radial fissures in the anulus fibrosus nucleus tissue is expelled. This material enters the intervertebral foramen and may compress the nerve root. It is astonishing that in a healthy disc no herniation occurs after a radial defect is made (Virgin, 1951). So a radial fissure is not the only condition for herniation. Probably a biochemical change, resulting in a decrease of viscosity of nucleus tissue is also needed.

\subsection{Existing models for swelling soft tissues}

Swelling theories in the literature can be roughly divided into two groups. The first one treats the tissue as a gel of charged macro-molecules. The swelling phenomena are explained by so called Donnan equilibria. The role of the fluid component is emphasized; the intrinsic stiffness of the solid phase is neglected. Generally viscous effects are excluded, these theories are one-dimensional. Due to the composition of the nucleus this approach may be suitable to model the response of nucleus tissue. The second group is an extension of existing non-equilibrium, 3-dimensional continuum theories of soft tissues to encompass swelling. The intrinsic stiffness of the solid component is explicitly modelled These models seem to be more appropriate to describe the behaviour of the anulus.

Different approaches/theories are used by several research groups. Especially the definition of the so-called swelling pressure is not unique. In the subsequent paragraphs their approach and conclusions are outlined. The conclusions given in the text are those of the referenced researchers. Generally these results differ from the results given in chapter 3 and 5.

\subsubsection{Donnan equilibria}

Consider two compartments separated by a semi-permeable membrane. Compartment I contains only solvent and low-molecular ions. The second compartment contains also charged macro-ions. The membrane is permeable for both the solvent and the low molecular ions. In soft tissues the negatively charged macromions are proteoglycans, the solvent is water and the low-molecular ions are usually $\mathrm{Na}^{+}$and $\mathrm{Cl}^{-}$. In this case the $\mathrm{Na}^{+}$ ions are the counter-ions while the $\mathrm{Cl}^{-}$are the co-ions. As $\mathrm{NaCl}$ is a uni-valent salt the number of moles equals the number of equivalences. Due to the imbalance of the chemical potential of the solvent and the ions, caused by the difference in ionic strength, a mass exchange of flutd and ions occurs. The fluid flow decays progressively as the 
hydrodynamic pressure in compartment II changes. The resulting pressure difference is called the osmotic pressure. In equilibrium the chemical potential of the solvent and the ions of both compartments must be equal. Assuming both compartments to be ideal dilute solutions and electrically neutral, the ion distribution and the osmotic pressure are given by (Richards, 1980):

electro neutrality:

$$
\bar{c}^{*}=\bar{c}^{-}=\bar{c} \quad c^{*}=c^{-*}+c^{p}
$$

equal chemical potential of the ions:

$$
\bar{c}^{*} \bar{c}^{-*}=c^{*} c^{-}
$$

equal chemical potential of the fluid:

$$
\pi=R T\left(c^{*}-\bar{c}^{+}+c^{-}-\bar{c}^{-}+C^{p g}\right)
$$

with $c$ the concentration in mole per unit fluid volume

suffixes,,$+- p g$ representing the cations, anions and proteoglycans respectiveIy

$c^{p g}$ the fixed charge density of the proteoglycans in equivalences per unit fluid wolume (FCD)

$C^{p e}$ the proteoglycan concentration in mole per unit tissue volume barred quantities are in compartment $\mathbb{l}$, unbarred in compartment II

$\pi$ the osmotic pressure

R universal gas constant

$T$ absolute temperature

Substitution of the electro neutrality $(2.1)$ in $(2.2)$ gives the ion distribution:

$$
\begin{aligned}
& 2 c^{*}=c^{p g}+\sqrt{\left(c^{p g}\right)^{2}+4 \vec{c}^{2}} \\
& 2 c^{-}=-c^{p g}+\sqrt{\left(c^{p g}\right)^{2}+4 \bar{c}^{2}}
\end{aligned}
$$

Due to charged macro ions the counter- and co-ion concentrations are not equal in both compartments. This unequal distribution is the Donnan effect. If the external salt con centration is kept constant the counter ion $\left(\mathrm{Na}^{+}\right)$concentration increases with increasing $\mathrm{FCD}$. The co ion $\left(\mathrm{Cl}^{-}\right)$concentration shows the opposite tendency. The Donuan effect is reduced by increasing the concentration of the salt. Due to the high molecular weight of the proteoglycans their concentration can be neglected compared to the cation and anion concentration. Hence, the Donnan osmotic pressure is caused by the imbalance of the solutes only. Substitution of the ion distribution (2.4) in the relation of the Donnan osmotic pressure yields:

$$
\pi_{d, n}=R T\left(c^{*}-\bar{c}^{+}+c^{-}-\bar{c}^{-}\right)=2 R T c\left(\sqrt{1+\frac{\left(c^{p b}\right)^{2}}{4 c^{2}}}-1\right)
$$

In the case of an excess of sall the term between brackets can be expanded in a binomial sericies: 


$$
\text { if }\left(\frac{c^{p g}}{2 \vec{c}}\right)^{2}<1: \pi_{d \omega n}^{i d t}=R T \frac{\left(c^{p g}\right)^{2}}{4 \vec{c}}
$$

This condition will never occur in vivo, it can only be created in vitiro. The ideal osmotic pressure is quadratic in the FCD. This indicates that the osmotic pressure is related to the second virial coefficient. The virial coefficients are analogue to the van der Walls theory for real gasses; the osmotic pressure of a real solution is expressed as:

$$
\pi=R T\left(\frac{c^{p g}}{M}+B\left(c^{p g}\right)^{2}+C\left(c^{p g}\right)^{3}+\cdots\right)
$$

with $\mathrm{M}$ the molecular weight of the proteoglycans

$\mathrm{B}, \mathrm{C}$ the virial coefficients depending on the external salt concentration

Only the first virial coefficient is independent of the external salt concentration. It is generally believed that the second virial coefficient accounts for three phenomena: the Donnan effect, the excluded volume effect and the ion-proteoglycans interactions and the self attraction of the proteoglycans. The excluded volume effect is a geometrical effect. The space occupied by one PG molecule is not available to a second molecule. This effect is negligible in a dilute solution but manifest in a concentrated solution. The Donnan effect and the ion interactions vanish in an excess of salt. In this case only the excluded volume effect contributes to the swelling pressure. An alternative expression for the osmotic pressure in a real solution is a modification of (2.3):

$$
\pi=R T\left\{\hat{\phi}\left(c^{+}+c^{-}+C^{p g}\right)=\bar{\phi}\left(\bar{c}^{+}+\bar{c}^{-}\right)\right\} \approx R T \phi\left\{\left(c^{+}+c^{-}\right)-\left(\bar{c}^{+}+\bar{c}^{-}\right)\right\}
$$

with $\phi$ the osmotic coefficient

$\hat{\phi}$ the osmotic coefficient in the tissue

$\bar{\phi}$ the osmotic coefficient in the external bath

The Donnan equilibrium theory $(2.5$ or 2.6 or 2.8$)$ is suitable to describe the osmotic pressure of extracted proteoglycans from cartilaginous tissues. Urban et al. (1979) used PG extracted from human hip, knee and intervertebral disc. The best-fit curve of their experimental results in physiological saline is given in fig. 2.3. In physiological saline the osmotic pressure shows a steep rise with increasing FCD. In the IVD the FCD varies from 0.08 to $0.15 \mathrm{meq} / \mathrm{g} \mathrm{H}_{2} \mathrm{O}$ in the anulus, while in the nucleus it varies from 0.18 to $0.35 \mathrm{meq} / \mathrm{g} \mathrm{H}_{2} \mathrm{O}$ (Urban and Maroudas, 1979). The total osmotic pressure range in the IVD is about 0.03 to $0.35 \mathrm{MPa}$. Under these conditions the Donnant osmotic pressure contributes for over $85 \%$ to the total osmotic pressure. This regardless of the origin, the size and the degree of aggregation of the PG. As the excluded volume effect is a geometrical effect it is independent of the ion concentration. Hence, the excluded volume effect in $0.15 \mathrm{M} \mathrm{NaCl}$ is approximated by the excluded volume effect in $1.5 \mathrm{M} \mathrm{NaCl}$. The excluded volume effect in $0.15 \mathrm{M} \mathrm{NaCl}$ is negligible for $\mathrm{FCD}$ less then $0.1 \mathrm{meq} / \mathrm{g}$ $\mathrm{H}_{2} \mathrm{O}$. For $\mathrm{FCD}$ above $0.3 \mathrm{meq} / \mathrm{g} \mathrm{H}_{2} \mathrm{O}$ the contribution is about 15 percent. Between 0.1 and $0.3 \mathrm{meq} / \mathrm{g} \mathrm{H}_{2} \mathrm{O}$ it increases linearly with FCD. Based on a least square fit the osmotic coefficient of $(2.8)$ is calculated as 0.830 . This value is in the same range as the one found for articular cartilage: 0.8 (Maroudas, 1975). It is clear from fig. 2.4 that (2.8) is capable of describing the osmotic pressure of extracted $\mathrm{PG}$. 


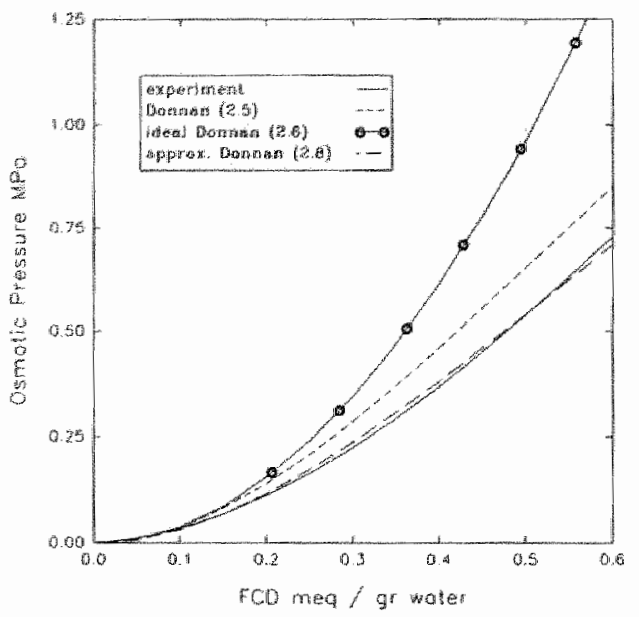

figure 2.4 The equilibrium osmotic pressure of extracted proteoglycans in contact with physiological saline as a function of fixed charge density (FCD). Comparison of experimental results (Urban et al., 1979) and different expressions for the Donnan osmotic pressure. Using the value $\phi=0.83$ in $(2.8)$ yields a good vit of the experimental data.

If tissue samples are used instead of extracted proteoglycans the situation is complicated due to the presence of the collagen network. In equilibrium the osmotic pressure is neutralised by the effective stress in the collagen network $P_{c}$ and any applied extemal pressure $\mathbf{P}_{\text {app }}$ :

$$
\pi=P_{c}+P_{a p p}
$$

Urban and Maroudas (1980) define the net swelling pressure $\mathrm{P}_{\mathrm{s}}$ as:

$$
P_{s}=\pi-P_{e}=P_{a p p}
$$

A more general definition is: the swelling pressure equals the difference between all effects contributing to swelling and the effective stress. If the tissue is loaded above $\mathrm{P}_{\mathrm{s}}$ fluid is expelled from the tissue leading to an increase of the PG concentration. Hence, the osmotic pressure rises, while the tension in the collagen network decreases. Fluid flow continues until the new net swelling pressure equals the applied pressure. In the absence of the collagen network the swelling pressure equals the osmotic pressure. Urban and McMullin $(1985,1988)$ have measured the swelling pressure of nucleus slices. If the FCD is based on the total amount of water in the tissue the swelling pressure is higher than the osmotic pressure of extracted PG for equal FCD. However by assuming that a part of the water is in the intra-fibrillar compartment they are able to shift the experimental curve to higher FCD; as mentioned in section 2.3 a value of $1.33 \mathrm{~g} \mathrm{H} \mathrm{H}_{2} \mathrm{O} / \mathrm{g}$ collagen is used. If the FCD is based on the amount of extra-fibrillar water the swelling pressure of tissue slices and the osmotic pressure of the extracted PG, omitting the collagen fibres, coincides. According to $(2.10)$ in physiological conditions the mechanical stress of the 
coarse collagen network in the nucleus is negligible compared to the osmotic pressure in the fluid. In wivo the osmotic pressure in the IVD is compensated by the body weight, ligament forces and the active muscle forces. The applied pressure is balanced by the fluid only. The sole role of the collagen network in the nucleus is to immobilize the proteoglycans.

\subsubsection{Continuum theories}

The continuum theories used to model soft tissues are based on a mixture approach. The tissue is viewed upon as a mixture of an elastic intrinsically incompressible solid and an intrinsically incompressible barotropic fluid. Momentum exchange between the two components is governed by the buoyancy force and the Stokes drag. This kind of biphasic theories, omitting swelling phenomena, are successfully used to study the mechanical response of articular cartilage (Mow et al., 1980, 1984), skin and subcutis (Oomens et al., 1987) and with a visco-elastic solid of the beating left ventricle (Huyghe et al., 1991, 1992). These researchers describe the mechanical behaviour of the tissue always relative to an existing osmotic pressure. If large deformations are involved the change in the proteoglycan concentration, resulting in change of the osmotic pressure, can not be neglected (Snijders et al., 1992). In a small deformation theory a constant osmotic pressure may be correct.

Silberberg (1982, 1989) formulates a multi-component theory which is specialised to a three component system: solid matrix, solvent and macro molecular solute. The driving forces for movement are the gradients of the chemical potential. These forces are counter balanced by frictional forces. The latter are proportional to the relative velocities. The macro molecular solute is uncharged: no ionic phenomena are involved.

Lanir (1987a) added to the momentum exchange between the fluid and solid matrix the concentration dependent part of the gradient of the chemical potential of the fluid: the concentration force. Compared to the above mentioned biphasic theories Darcy's law is modified. The driving force for relative fluid flow decreases with the concentration force. Limiting to small deformations Lanir (1987ab) showed that for both confined and unconfined compression the tissue is stiffer with a concentration force than without. Even if the collagen fibres buckle and their stiffness is reduced, the tissue is able to carry the applied load by the fluid component. The model is capable of predicting experimental observations qualitatively. Unfortunately a quantitative confrontation with experimental results is omitted. The role of diffusible small ions species is not incorporated.

In analogy to linear thermo-elasticity Myers et al (1984) added to the constitutive relation of the solid matrix a chemical expansion stress (called the chemical contraction stress by the authors), which is proportional to the local salt concentration. The latter is calculated by the standard diffusion equation. The PG concentration is not incorporated into the model. Free swelling experiments on bovine articular cartilage shows a linear relationship between the contraction stress and the concentration up to $0.2 \mathrm{M} \mathrm{NaCl}$. The driving pressure in $0.15 \mathrm{M} \mathrm{NaCl}$ is $0.145 \mathrm{MPa}$ which is consistent with the values of Maroudas et al. (1985). The calculated response to isometric contraction of a thin strip due to a step in the external salt concentration shows a qualitative good agreement with the actual experiment.

In a similar way Eisenberg and Grodzinsky $(1985,1987)$ introduced both a salt dependent chemical-expansion stress and stress strain relation. Both the stress-strain relation 
and the chemical expansion stress decrease monotonically with increasing salt concentration. The local salt concentration is also calculated with the standard diffusion equation. For the initial and boundary conditions the Donnan equilibrium concentration is used. Confined compression experiments on bovine articular cartilage shows that the chemical expansion stress is negligible for $\mathrm{NaCl}$ concentrations above $0.2 \mathrm{M}$. This is in contradiction with the results of Maroudas et al. (1985): who found a response for higher $\mathrm{NaCl}$ concentrations. The stress strain relation is independent of the concentration above $0.5 \mathrm{M}$. The calculated response is in qualitative agreement with the experiment. The ratio of the time constants for diffusion and fluid flow differs from the one obtained by Myers et al. (1984). For both models the origin of the stiffness and the swelling is sought in the electrostatic repulsion forces between the charged groups on the $P G$ chains. Increase of the $\mathrm{NaCl}$ concentration results in electrostatic shielding: stiffness and swelling decrease. In contrast with the Donnam theory swelling is associated with the solid matrix. In micro continuum models for the swelling of polymer gels the volume change is calculated on an energy basis (Richards, 1980). The swelling, caused by the penetration of the solvent (fluid), results in a better mixing. Hence, the free energy decreases which is compensated. by the increase of free energy stored in the deformation of the polymer network. In equilibrium the free energy is minimum. If the gell consists of charged macromolecules. the free energy is additionally decreased by an ionic contribution: the Donnan effect. In most charged polymer networks the latter is dominant over the mixing contribution (Ruchards, 1980). For both thermo-elastic models the osmotic contribution in the definition of the swelling stress $(2.10)$, is replaced by the chemical expansion stress. The experimental specimen were obtained from the same location and animal. However the experimental results show a complete different qualitative tendency (fig 2.5). The reason for this discrepancy is unclear.

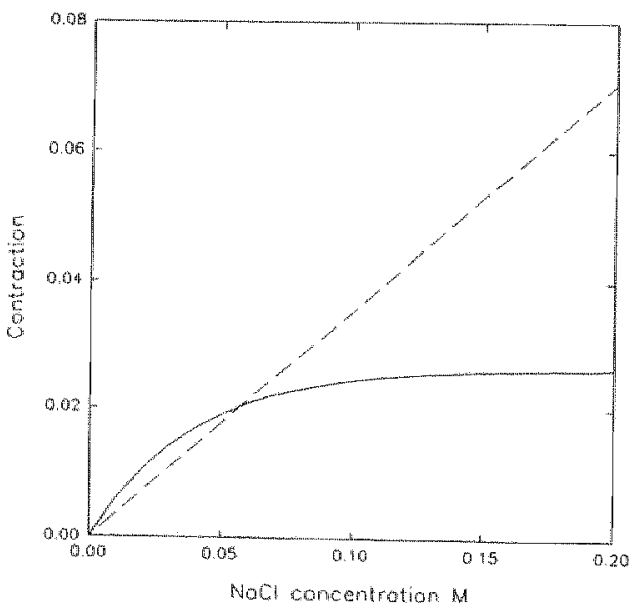

figure 2.5 Contraction from the hypotonic reference state for bovine articular cartilage, using a thermo-elasticity analogy for the swelling phenomena. Experimental results: solid line: Myers, 1984; dashed line: Eisenberg,
1985 . 
Although the models of Myers et al. (1984) and Eisenberg and Grodzinsky (1985, 1987) could explain their experimental observations, their bases are more phenomenological than structurall.

Mow and coworkers (Mow et al. 1990, Lai et al. 1991) explicitly model the ionic component. Hence, the resulting theory is triphasic. The ionic component is treated as a second fluid. Starting from the general balance laws constitutive restrictions based on the entropy principle are derived. The total mixture stress is composed of three parts. the fluid pressure, the elastic stress and the chemical expansion stress. The latter is associated with the solid matrix and is due to repulsive forces on the PG chains. The stress-strain relation is salt dependent. Three components contribute to the chemical potential of the fluid: the hydrodynamic pressure, the ion concentration and the volume change of the solid matrix. Only the latter two contribute to the chemical potential of the ions. The resulting driving forces for swelling are: the Donnan osmotic pressure (2.8), the osmotic pressure due to the presence of the $\mathrm{PG}$, the excluded volume effect due to the volume change of the solid matrix and the chemical expansion stress. All these forces contribute to the osmotic pressure as defined in $(2.10)$, they are balanced by elastic deformations and the applied external load. Based on free swelling experiments on bovine articular cartilage the material parameters are determined. Using these material values some simple thought experiments in a confined compression set-up are analyzed. First keep the tissue at its hypertonic reference state and change the $\mathrm{NaCl}$ concentration of the external bath. The Donnan osmotic pressure contributes for $50 \%$ to the total swelling pressure. The other $50 \%$ originates from the chemical expansion stress. Second consider a sample in equilibrium with a $0.15 \mathrm{M} \mathrm{NaCl}$ solution. The total swelling pressure contributes for 20 $\%$ to the total stiffness, the other $80 \%$ is the intrinsic stiffness of the solid matrix. Both results are in contradiction with the results of Urban et al. (1979) and Maroudas et al (1985). Their conclusions are twofold: the Donnan osmotic pressure contributes for over $85 \%$ to the swelling pressure; the apparent stiffness originates from the change of the osmotic pressure with hydration and is associated with the fluid.

It is clear from the discussion above that no uniform results are obtained by different researchers. The differences are not only quantitative of nature but also qualitative. To which extend does the fluid contribute to the load bearing of intervertebral disc tissue? What is the role of the osmotic effect in the load transmission?

\subsection{References}

Bogduk, N., Twomey, L.T. Clinical anatomy of the lumbar spine. Churchill Livingstone, 1987.

Comper, W.D., Laurent, T.C. Physiological function of connective tissue polysaccharides. Physiological Reviews, 58:255-315, 1978 .

Eisenberg, S.R. Grodzinsky, A.J. Swelling of articular cartilage and other connective tissues: electromechanochemical forces. J. Orthop. Res., 3:148-159, 1985 .

Eisenberg, S.R., Grodzinsky, A.J. The kinetics of chemically induced nonequilibrium swelling of articular cartilage and comeal stroma. J Biomech. Engng., 109:79-89, 1987. 
Grodzinsky, A.J. Electromechanical and physicochemical properties of connective ussue. CRC Critical Reviews in Bhomed. Engng;, 9.133 199, 1983.

Huyghe, I.M., Cumpen, D.H. van, Ants, T, Heathar, R.M. A two phase finite element model of the diatolic left ventricle. I. Biomed., 24:527-538, 1990 .

Huyghe, J.M., Arts, T., Campen, D.H. van, Reneman, R.S. Porous madium finite element model of the beating left ventride. Am. J. Physiol., 262:1256-1267, 1992.

Inowe, H. Threse-dimencional architecture of lumbar intervertebral discs. Spine, 6:139-146, 1981.

Johnstone, B., Urban, I.P.G., Roberts, I., Menage, I. The flud contents of the thuman interveriebral disc. Spine, $17,412-416,1902$.

Lai, W.M., Hou, J.S., Mow, V.C. A triphasic for the swelling and deformation behaviours of articular cartilage. I. Biomech. Engng, 113:245-258, 1991 .

Lanir, Y. Biorheology and thid flux in swelling tissues. I Bicomponent theory for small deformations, including concentration wfects. Biorheology, 24:173-187, 1987a.

Lanir, Y. Biorheology and fluid flux in swelling tissues. II Analysis of unconfined compressive response of transwersely isotropic cartilage disc. Biorheology, 24:189-205, $1987 \mathrm{~b}$.

Marchand, Fi, Ahmed, A.M. Investigation of the laminate structure of lumbar dise anulus fibrosus. Spine, $15: 402-410,1990$.

Maroudas, A. Biophysical chemistry of cartilaginous tissues with special reference to solute and fluid transport. Biorheology, 12:233248, 1975 .

Maroudas A. ZZiv, 1., Weisman, N., Venn, M. Studies of bydration and swelling pressure in normal and osteoarthritic cartilatge. Biorheology, 22:159-169, 1985.

Mow, V.C., Kuei, S.C., Lai, W.M. Biphasic creep and stress relaxation of articular cartilage in compression. J. Biomech. Engng., 102:73-84, 1980.

Mow, V.C., Holmes, H.H., Lai, W.M., Fluid transport and mechanical properties of articular cartilage: a roview. 3. Biomech, $17,377-394,1984$

Mow, V.C. Lai, W.M., How, J.S. "Triphasic theory for swelling properties of hydrated charged soft biological tissues. Appl. Mech. Rev., 43:134-141, 1990.

Myers, E.R., Lai, W.M. Mow, V.C. A continutm theory and experiment for the lon-induced swelling behaviour of articular cartilage. J. Biomech. Engng., 106:151-158, 1984.

Oomens, C.W.J., Campan, D.H. wan, Grootenboer, H.J. A mixture approach to the mechanics of skin. J. Biomech, $20: 877-885,1987$.

Richards, E.G. An introduction to physical properties of large molecules in solution. Cambridge University Press, Cambridge, 1980 .

Roberts, S., Mertage, J., Urban, J.P.G. Biochenical and structural properties of the cartilage end-plate and its retation to the intervertebral disc. Spine, 14:166-174, 1989.

Silbertherg, A. The mectanics and thermodynamics of separation flow through porous, molecular disperse, 
solid media. The Poisewille Lecture 1981. Biorheology, 19.111-127, 1982.

Silberberg, A. Transport through deformable matrices. Biorheology, 26:291-313, 1989.

Snjders, H., Huyghe, J., Willenss P., Drost, M. Janssen, J., Huson, A. A mixture appronoh to the mechanics of the human intervertebral disc. in: Mechanics of swelling: from clays ro liwing ofls and tissues, (ed. Karalis, T.D.), 545-558, 1992.

Urban, J.P.G., Maroudas, A., Bayliss, M.T. Dillon, J. Swelling pressures of proteoglyoans at the concentrations found in cartilaginous tissues. Biorheology, $16: 447-464,1979$.

Urban, J., Maroudas, A. The measurement of fixed charge density in the intervartebral disc Biochamioa at Biophysica Acta, $586: 166-18,1979$.

Urban, J, Maroudas, A. Measurement of swelling pressure and fluid flow in the intervertebral disc with reference to creep. Proc. Inst. Mech. Eng., C132/80:63-69, 1980.

Urban, J.P.G., McMullin, J.F. Swelling pressure of the intervertebral disc: influence of proteoglycan and collagen contents. Biorheology, 22:145-157, 1985.

Urban, J.P.G., McMullim, J.F. Swelling pressure of the lumbar intervertebral discs: influence of aye, spinal level, composition and degeneration. Spine, $13: 179-187,1988$.

Virgin, W.1. Experimental investigations into the physical properties of the intervertebral disc. J. Bone Joint Surg. (Br.), 33B:607-611, 1951.

White, A.A., Panjabi, M.M. Clinical biomechanics of the spine. J.B. Lippicott Company, Philadelphin, 1978 .

Williams, P.L., Warwick, R., Dyson, M., Bannister, L.H. Gray's Anatomy. Churchill Livingstone, Edinburgh, 1989. 

Deformation of intervertebral disc tissue can be achieved either by mechanical or chemical loading. The overall response is the result of four different, simultaneously occurring physical processes. (1) The diffusion of mobile ions. (2) Specific binding of ions to the proteoglycan-chain. (3) Large deformation of the fibre network and the ground substance with relative fluid flow. (4) Readjustment of the local electrical fields within the matrix. On the basis of the estimation of time constants (section 3.0) we developed a mechanical model for intervertebral disc tissue including "osmotic" effects, ion diffusion and large matrix deformation combined with relative fluid flow. In order to describe this complex behaviour we make use of the theory of mixtures. Starting with the kinematic relationships (section 3.1) and the general balance laws (section 3.2) constitutive restrictions based on the entropy principle are derived (section 3.3). In section 3.4 the basic equations are summarized. In section 3.5 the consequences of the presented triphasic theory are discussed by means of one-dimensional swelling. 


\subsection{Introduction}

Intervertebral disc tissue consists of a collagen and elastim fibre network imbedded in a hydrated proteoglycan (PG) matrix. Nutrients and ions are dissolved within the tissue. Because of the entanglement of the $P G$ matrix with the fibre network only the interstitial fluid and the ions can move freely. Because the $\mathrm{PG}$ are ionized and relatively immobile, "osmotic" effects are important. Deformation of the tissue can be achieved either by mechanical or chemical loading. The overall response which occurs in cartilaginous tissues, is the result of four different, simultaneously occurring physical processes, each with a different time constant. (1) The diffusion of mobile ions. A typical value for the time constant of diffusion for intervertebral disc tissue is $1500 \mathrm{~s}$ (specimen thickness 1 mm) (Maroudas, 1975). This time constant is defined as the quotient of the square of the sample thickness and the diffusion coefficient. (2) Specific binding of ions to the PGchains, resulting in a limitation of the diffusion, will be neglected. This is valid for the diffusion of $\mathrm{NaCl}$, the ions we used in our experimental set-up (Maroudas, 1975). (3) The large deformation of the fibre network and the ground substance with relative fluid flow into or out of the tissue. In the limiting case of small deformation a typical value of the time constant is $1200 \mathrm{~s}$ (specimen thickness $1 \mathrm{~mm}$ ) (Best et al., 1989). In chapter 5 values of $3200 \mathrm{~s}$ and $900 \mathrm{~s}$ are found in the axial and radial direction respectively. This time constant is defined as the quotient of the square of the sample thickness and the product of the permeability and the aggregate modulus. (4) Readjustment of the local electrical fields within the matrix, due to the volume change of the PG-matrix or the change of ion content. Typical values are $\mathbb{1}$ ns (Grodzinsky et al., 1981). This time constant is defined as the quotient of dielectric constant and the conductivity of the interstitial fluid. Hence this time constant is independent of the sample thickness. The charge relaxation is never rate-limiting, because of the small time constant compared to the time constants of diffusion and fluid flow. Based on the estimation of the time constants a mechanical model for intervertebral disc tissue including "Donnan osmotic" effects, ion diffusion and large matrix deformation combined with relative fluid flow is developed.

Suffix $\alpha$ stands for an arbitrary constituent, while the suffixes $f, s$ and $i$ stand for respectively fluid, solid and ions.

\subsection{Kinematics}

The theory presented, is based on the theory of mixtures by Bowen (1980) and Müller (1985). The basic assumption is that the mixture may be viewed upon as a superposition of single continua (phases), each following its own motion. This implies an averaging procedure within an elementary volume. During this averaging procedure both mathematical and physical requirements should be complied with to define averaged macroscopic quantities in terms of the microscopic quantities. Details of such an averaging procedure can be found in the literature (Whitaker, 1969; Slattery, 1972; Hassanizadeh and Gray, 
1979). Every microscopic property $\gamma$ has a 'true' and a 'apparent' macroscopic walue. The true property of the ath constituent $\gamma^{*}$ is defined as the volume integral of $\gamma^{n}$ over the averaging volume divided by the true volume $v^{\text {ex }}$. The apparent property or bulk volume averaged value $\bar{\gamma}^{\alpha}$ is integrated in the same way but is divided by the mixture

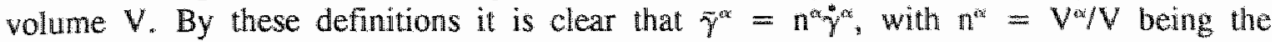
volume fraction of constituent $a$. Hence, by means of the averaging procedure each constituent is virtually present everywhere in the mixture volume. Unless otherwise stated all properties are apparent properties. It is assumed that at any time there exists an oneto-one continuous and differentiable relationship between the current position vector $\bar{x}{ }^{\text {ix }}$ and the initial position vector $\vec{x}_{0}^{\infty}$ of a constituent:

$$
\vec{x}=\vec{x}^{* t}=\dot{\Psi}^{\alpha}\left(\vec{x}_{0}^{*}, t\right)
$$

We assume that within each averaging volume the different constituents are homogeneously distributed. Therefore all current position vectors $\vec{x}^{\text {ix }}$ coincide for all different phases; which is generally not true for the corresponding reference vectors $\overrightarrow{x_{0}^{\circ}}$.

For the definition of the material time derivative it is important with which constituent the observer is moving:

$$
(\dot{\gamma})_{a c}=\frac{D^{\alpha}}{D t} \gamma=\frac{\partial \gamma}{\partial t}+\vec{\nabla} \gamma \circ \nabla^{* a}
$$

$\vec{\nabla}$ represents the gradient operator with respect to the current configuration and is constituent independent; $\vec{v}^{t t}$ is the velocity of the $\alpha$ th constituent. The $\alpha$ th material time derivative may be transformed to the material derivative following another arbitrary constituent $\beta$ by:

$$
\frac{D^{\beta}}{D t} \gamma-\frac{D^{\alpha}}{D t} \gamma=\left(\vec{v}^{\beta}-\vec{v}^{\alpha}\right) d \vec{\nabla} \gamma
$$

Because the constituents are regarded as continua all other kinematic quantities, such as the velocity or the deformation gradient are formulated in the same way as in a single continuum theory. Therefore their definition is omitted here.

\subsection{Balance laws}

As a result of the averaging procedure each constituent is viewed upon as a continuum following its own motion. Therefore, it is possible to derive balance laws for each constituent. The balance laws are identical with the balance laws of a single phase continuum except for interaction terms. These terms account for the presence of the other constituents. The balance laws of the mixture can be derived in two ways. Firstly by treating the mixture as a single continuum: the mixture does not know it is a mixture. Secondly by adding the balance laws of the constituents. By comparing these two results it is possible to find expressions for the mixture properties as a function of the constituents" properties. These expressions are not unique (Bowen, 1975, 1980; Atkin and Craine, 1976; Müller, 1973, 1985). For physical reasons the restrictions on the interaction terms are unique: their sum is zero, on the mixture level there are no interactions. 


\subsubsection{Mass balance}

The local mass balance for the oth constituent is given by:

$$
\frac{D^{\alpha}}{D t} \rho^{\alpha}+\rho^{\alpha} \ddot{\nabla} \circ \vec{v}^{\alpha}=e^{\alpha} \text { or } \dot{\rho}^{\alpha}+\rho^{\alpha} \vec{\nabla} \circ \vec{v}{ }^{\alpha}=e^{*}
$$

The mass supply from the other constituents is accounted for by the interaction term $\mathrm{e}^{\mathrm{r}}$. If chemical reactions or phase transitions occur, this term is non zero. However, the mass balance of the mixture leads to the restriction:

$$
\sum_{x} e^{\alpha}=0
$$

Within intervertebral disc tissue three different constituents are distinguished: solid, fluid and ions. The elastin and collagen fibres and the proteoglycan ground matrix are considered to be an intrinsically incompressible solid. This means that the true density of the solid phase $\Gamma^{\text {is }}$ is constant in time. The second constituent is the fluid, which is also intrinsically incompressible. The fluid and solid constituent are immiscible. Volume change of the mixture is achieved only by squeezing fluid out of the tissue. The third constituent consists of the small nutrients and ions. Although many different ions and nutrients are involved, they are treated as one constituent: the ion phase. The latter consists of two species: the anions (negatively charged) and the cations (positively charged). Both the counter-ions, associated with the ionized PG-chains and the free mobile ions contribute to the total cation concentration. In fact the ion constituent is a mixture by itself. It is treated as one single phase of a monovalent salt to reduce the number of variables involved. As sodium chloride is used in the experiment described in chapter 5, the salt is often referred to as $\mathrm{NaCl}$. The ionic phase is also assumed intrinsically incompressible and the ionic species are immiscible with both the fluid and solid. The densities of the fluid and solid constituent $\left(\rho^{\mathrm{f}}\right.$ and $\left.\rho^{s}\right)$ are expressed as a function of the volume fractions $\left(n^{f}\right.$ and $\left.n^{g}\right)$ and the intrinsic (true) density ( $\Gamma^{f}$ and $\Gamma^{s}$ ); the density of the ionic species $\left(\rho^{+}\right.$and $\left.\rho^{-}\right)$is expressed as a function of the concentration per unit tissue volume $\left(\mathrm{C}^{+}\right.$and $\left.\mathrm{C}^{-}\right)$and the mole mass $\left(\mathrm{M}^{*}\right.$ and $\left.\mathrm{M}^{-}\right)$; the ion velocity is mass averaged:

$$
\begin{array}{ll}
\rho^{s}=m^{*} & \rho^{f}=n^{f} \mathrm{~T}^{t} \\
\rho^{*}=M^{+} C^{*} & \rho^{-}=M^{-} C^{-} \\
\rho^{i} & =\rho^{*}+\rho^{-} \\
\rho^{i} \vec{v}^{i} & =\rho^{*} \vec{v}^{*}+\rho^{-\vec{v}^{-}}
\end{array}
$$

If no external electrical field is applied to the tissue, charge neutrality is maintained while the electrical current density equals zero. In this case the velocity of the cations and the anions are related to each other and can be calculated if the ion velocity $\vec{v}^{i}$ is known. The volume of the ions is neglected compared to the volume occupied by the solid and the fluid. As the tissue remains hydrated the sum of the volume fractions equals unity: $n^{s}+$ $n^{\prime \prime}=1$. No chemical reactions take place $\left(\mathrm{e}^{\alpha}=0\right)$, so the mass balances for the three constituents are: 


$$
\begin{aligned}
& \text { solid : } \hat{n}^{x}+n \vec{\nabla} \nabla \vec{\nabla} \vec{y}^{\mathbb{4}}=0 \\
& \text { fluid : } \dot{n}^{f}+n+\vec{\nabla}_{\circ} \vec{\psi}^{f}=0 \\
& \text { ions: : } \dot{\rho}^{i}+\rho^{i} \vec{\nabla} \circ \vec{v}^{i}=0
\end{aligned}
$$

The ionized proteoglycans affect the equations in two ways: their volume contribution is incorporated in the mass balance of the solid while their electrical charge influences the ion flux. Summation of the mass balance of the solid and fluid (3.7.1 and 3.7.2) and eliminating the local time derivative yields the mass balance of the mixture:

$$
\vec{\nabla} \circ \vec{v}^{s}+\vec{\nabla} \circ\left(n^{f}\left(\vec{v}^{f}-\vec{v}^{\prime}\right)\right)=0
$$

\subsubsection{Balance of momentum}

The balance of momentum for the $\alpha$ th constituent is given by:

$$
\rho^{\alpha} \frac{D^{\alpha}}{D t} \vec{v}^{\alpha}=\vec{\nabla} \circ g^{\alpha}+\rho^{\alpha} \vec{q}^{+\alpha}+\vec{\pi}^{\alpha}
$$

with $\underline{g}^{\text {sx }}$ partial Cauchy stress tensor

$$
\frac{\bar{q}^{\alpha}}{\pi^{\alpha}} \text { mody force per unit mass }
$$

The restriction on the interaction terms, found by making use of the momentum balance for the mixture, is given by:

$$
\sum_{\alpha}\left(\vec{\pi}^{\alpha}+e^{\alpha} \vec{v}^{\alpha}\right)=\overrightarrow{0}
$$

Because we consider only slow deformation rates, inertial effects are neglected. The body force is also neglected. This results into:

$\begin{array}{lll}\text { solid } & : \vec{\nabla} \circ \underline{Q}^{s}+\vec{\pi}^{s} & =\overrightarrow{0} \\ \text { fluid } & : \vec{\nabla} \circ \underline{\Omega}^{f}+\vec{\pi}^{f} & =\overrightarrow{0} \\ \text { ions } & : \vec{\nabla} \circ \underline{g}^{l}+\vec{\pi}^{l} & =\overrightarrow{0} \\ \text { interaction }: \vec{\pi}^{s}+\vec{\pi}^{f}+\vec{\pi}^{l} & =\overrightarrow{0} \\ \text { mixture }: \vec{\nabla} \circ \underline{g}^{s}+\vec{\nabla} \circ \underline{g}^{f}+\vec{\nabla} \circ \underline{g}^{l} & =\overrightarrow{0}\end{array}$

\subsubsection{Balance of moment of momentum.}

The balance of moment of momentum for the $\alpha$ th constituent is given by:

$$
\vec{n}^{\alpha}-\vec{s}^{\alpha}=\overrightarrow{0}
$$
with $\quad \overrightarrow{\mathrm{m}}^{\alpha}$ the moment of momentum supplied by the other constituents
$\vec{s}^{\alpha}$ axial vector of the skew-symmetric tensor $\underline{q}^{\alpha x}-\left(\underline{\sigma}^{\alpha}\right)^{\mathrm{c}}$

A non-zero $\overrightarrow{\mathrm{m}}^{\alpha}$ implies a non-symmetric partial Cauchy stress tensor. Until proof of the 
contrary, we assume $\bar{m}^{\alpha}$ equal to zero, and hence the partial Cauchy stress to be symmetric. Often these continua are called 'simple' mixtures.

\subsubsection{Balance of energy or first axiom of thermodynamics}

The energy ballance for the $\alpha$ th constituent is given by:

$$
\rho^{a} \dot{\varepsilon}^{\alpha}=\underline{Q}^{\alpha}: \vec{V}^{*} \vec{v}^{\alpha}-\vec{\nabla} \cdot \vec{j}^{a}+\rho^{a} r^{\alpha}+g^{\alpha}
$$

with $\vec{j}$ theat flux

$e^{\alpha} \quad$ specific internal energy

$r^{*} \quad$ external specific heat supply

$\mathrm{g}^{\text {si }} \quad$ heat supply by the other constituents

Again the balance of the mixture is used to find the restriction on the interaction terms:

$$
\sum_{a}\left\{g^{\alpha}+\vec{\pi}^{\alpha}+\vec{v}^{\alpha}+e^{\alpha}\left(\varepsilon^{\alpha}+\frac{1}{2} \vec{v}^{\alpha} \omega \vec{v}^{\alpha}\right)\right\}=0
$$

\subsubsection{Entropy inequality or second axiom of thermodynamics}

The mass, momentum and energy balance form a set of coupled differential equations. Even with the right initial and boundary conditions this set of equations cannot be solved because the constitutive behaviour is unknown. The constitutive theory for mixtures based on the second axiom of thermodynamics (entropy inequality) has been a main point of discussion in the literature: the main dispute concerns the relationship between the mixture properties and the constituents properties. It can be shown that for 'simple' mixtures no essential differences result from application of either theory (Atkin and Craine, 1976). In this context the formulation for the entropy inequality according to Müller (1985) is used. For each constituent it is hypothesized that:

$$
\rho^{\alpha} \dot{\eta}^{\alpha}+\vec{\nabla} \frac{\vec{j}^{\alpha}}{T^{\alpha}}+\frac{\rho^{\alpha} r^{\alpha}}{T^{\alpha}}=\xi^{\alpha}
$$

with $\eta^{\alpha}$ specific entropy

$\xi^{\text {six }}$ entropy production

$\mathrm{T}^{\mathrm{ar}}$ the absolute temperature

For the purpose of deriving the restrictions on the constitutive relations, it is unnecessary to include the external heat supply, because these restrictions are unaffected by the presence of this supply. Because the entropy production may be caused by entropy exchange with other constituents, the classical demand of a positive entropy production is not claimed for each individual constituent. Their sum, which is the total entropy production on the mixture scale, must be positive or zero:

$$
\sum_{\alpha}\left(\rho^{\alpha} \dot{\eta}^{\alpha}+\vec{\nabla}_{0} \frac{\vec{j}^{\alpha}}{T^{\alpha}}+\frac{\rho^{\alpha} r^{\alpha}}{T^{\alpha}}\right)=\sum_{a} \xi^{\alpha} \geq 0
$$

By means of the introduction of the Helmhoilz free energy $A\left(A=\epsilon_{\epsilon}-T \eta\right)$ it is possible to combine the first and second axiom of thermodynamics. All experimental conditions which are considered in a following paragraph and the in vivo situation are assumed 
isothermal: each constituent has the same constant temperature. With no chemical reactions $\left(e^{a}=0\right)$ the resulting entropy inequality for the mixture is given by:

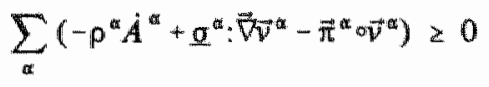

The constitutive relationships should be such that for any state of the mixture, complying with the kinematic restrictions $(3.7 .3)$ and $(3.8)$ the inequality $(3.17)$ holds. When incorporating these restrictions into the inequality we find:

$$
\begin{aligned}
& -\rho^{i} \dot{A}^{i}-\rho^{s} \dot{A}^{s}-\rho^{f} \dot{A}^{f}+\alpha^{i}: \vec{\nabla} \vec{v}^{i}+a^{f}: \vec{\nabla}^{f} \vec{v}^{f}+\alpha^{s}: \vec{\nabla} \vec{v}^{s} \\
& -\vec{\pi}^{i} \circ \vec{v}^{i}-\vec{\pi}^{f} \circ \vec{v}^{f}-\vec{\pi}^{s} \circ \vec{v}^{s} \\
& +\lambda\left\{\vec{\nabla}^{\prime} \circ \vec{v}^{s}+\vec{\nabla} \circ\left(n^{f}\left(\vec{v}^{f}-\vec{v}^{s}\right)\right)\right\} \\
& +\kappa\left(\dot{\rho}^{i}+\rho^{i} \vec{\nabla} \circ \vec{v}\right) \geq 0
\end{aligned}
$$

The Lagrange multipliers $k$ and $\lambda$ are unknown and have to be identified by the elaboration of the extended entropy inequality (3.18).

\subsection{Constitutive restrictions}

By means of the extended entropy inequality (3.18) it is possible to find restrictions on the constitutive relations for the dependent variables:

$$
A^{s}, A^{f}, A^{i}, \underline{Q}^{s}, \underline{q}^{f}, \underline{Q}^{i}, \vec{\pi}^{s}, \vec{\pi}^{f}, \vec{\pi}^{i}, \lambda, \kappa
$$

This list contains simply all unknowns appearing in the balance laws. The dependent variables may be functions of the primary unknowns: mass densities, displacements or their derivatives. Based on experimental results a set of independent variables has to be chosen. The constitutive restrictions that will be found, are only valid and consistent within this framework. By choosing another set different results can be obtained. The number of independent variables is limited by practical reasons. The choice should be an optimum for realistic physical modelling, experimental accessibility and mathematical complexity. For the set of independent variables we choose:

$$
\mathrm{E}^{s}, \rho^{f}, \vec{\nabla} \rho^{t}, \rho^{i}, \vec{\nabla} \rho^{i}, \vec{\omega}, \vec{u}
$$

with

$$
\begin{aligned}
& \vec{\omega}=\vec{v}^{\prime}-\vec{v}^{\prime} \\
& \vec{v}=\vec{v}^{s}-\vec{v}^{\prime} \\
& \vec{u}=\vec{v}^{r}-\vec{v}^{s}=\vec{\omega}-\vec{v}
\end{aligned}
$$

The Green-Lagrange strain tensor $\mathrm{E}^{\mathrm{a}}$ accounts for the deformation of the solid constituent. The mass density $\rho^{s}$ is solely determined by the reference value $\rho_{0}^{s}$ and the volume change of the porous solid. This volume change can be expressed in terms of the invariants of $\mathrm{E}^{s}$. Therefore $\rho^{8}$ is not included in the list of independent variables. The fluid constituent is assumed to be macroscopicaliy non viscous; hence the deformation rate tensor has not been chosen as an independent variable. The microscopic viscous effect is incorporated by 
the relative velocity $\vec{u}$. Diffusion effects are included by means of the relative velocities $\vec{\omega}$ and $\vec{v}$. The buoyancy effect as defined by Bowen (1980) is included by the gradient of the fluid density. The ion density and the gradient of the ion density allow for modelling ionic effects.

The principle of equipresence requires that all dependent variables depend on all independent variables. By postulating:

$$
\begin{aligned}
& A^{i}=A^{f}\left(E^{s}, \rho^{f}, \rho^{f}\right) \\
& A^{s}=A^{s}\left(E^{s}, \rho^{f}, \rho^{f}\right) \\
& A^{f}=A^{f}\left(E^{s}, \rho^{f}, \rho\right) \\
& \vec{\pi}^{f}=\vec{\pi}^{f}\left(E^{s}, \rho^{f}, \rho^{f}, \vec{\nabla} \rho^{f}, \vec{\nabla} \rho^{i}, \vec{v}, \vec{\omega}\right) \\
& \vec{\pi}^{s}=\vec{\pi}^{s}\left(E^{s}, \rho^{f}, \rho^{t}, \vec{\nabla} \rho^{f}, \vec{\nabla} \rho^{i}, \vec{v}, \vec{\omega}\right) \\
& \vec{\pi}^{i}=\vec{\pi}^{i}\left(E^{w}, \rho^{f}, \rho^{i}, \vec{\nabla} \rho^{f}, \vec{\nabla} \rho^{i}, \vec{v}, \vec{\omega}\right) \\
& \kappa=\kappa\left(E^{s}, \rho^{f}, \rho^{i}, \vec{\nabla} \rho^{f}, \vec{\nabla} \rho^{i}, \vec{v}, \vec{\omega}\right) \\
& \lambda=\lambda\left(E^{s}, \rho^{f}, \rho^{i}, \vec{\nabla} \rho^{f}, \vec{\nabla} \rho^{i}, \vec{v}, \vec{\omega}\right)
\end{aligned}
$$

this principle is approximated. The reason for this approximation is twofold. First, it is assumed that the density gradients and the relative velocities only influence the momentum interaction. Second, the model is still realistic while the complexity of the equations obtained is reduced. The constitutive relations (3.22) should not violate the extended entropy inequality (3.18). The chain rule of differentiation is used to evaluate the time derivatives:

$$
\begin{aligned}
& \dot{A}^{i}=\frac{\partial A^{i}}{\partial E^{s}}: \frac{D^{i}}{D t}\left(E^{s}\right)+\frac{\partial A^{i}}{\partial \rho^{i}} \dot{\rho}^{i}+\frac{\partial A^{i}}{\partial \rho^{f}} \frac{D^{i}}{D t}\left(\rho^{f}\right) \\
& \dot{A}^{f}=\frac{\partial A^{f}}{\partial E^{s}}: \frac{D^{f}}{D t}\left(E^{s}\right)+\frac{\partial A^{f}}{\partial \rho^{\prime}} \frac{D^{f}}{D t}\left(\rho^{j}\right)+\frac{\partial A^{f}}{\partial \rho^{f}} \dot{\rho}^{f} \\
& \dot{A}^{s}=\frac{\partial A^{s}}{\partial E^{s}}: \dot{E}^{s}+\frac{\partial A^{s}}{\partial \rho^{f}} \frac{D^{s}}{D t}\left(\rho^{f}\right)+\frac{\partial A^{s}}{\partial \rho^{i}} \frac{D^{s}}{D t}\left(\rho^{t}\right)
\end{aligned}
$$

The material time derivative of a property of a constituent different from the phase associated with the derivative is transformed by (3.3). Making use of the symmetry of $\underline{E}^{\sharp}$ and $2 \mathrm{~A}^{*} / \partial \mathrm{E}^{\mathrm{a}}$ it can be shown that:

$$
\begin{gathered}
\frac{\partial A^{s}}{\partial E^{s}}: \dot{E}^{s}=E \circ \frac{\partial A^{s}}{\partial E^{s}} \circ E^{c}: \vec{\nabla} \vec{\nabla}^{s} \\
\frac{\partial A^{\alpha}}{\partial \underline{E}^{s}}:\left[\vec{\nabla} E^{s} \cdot\left(\vec{v}^{\alpha}-\vec{v}^{s}\right)\right]=\left(\vec{\nabla}^{\alpha}-\vec{v}^{s}\right) \frac{\partial A^{\alpha}}{\partial \underline{E}^{s}}: \cdot \vec{\nabla} E^{s}
\end{gathered}
$$

with $F$ the deformation tensor

Substituting these results and (3.11.4) in the extended entropy inequality (3.18) and after 
rearranging, resulis into:

$$
\begin{aligned}
& {\left[-\rho^{s} \frac{\partial A^{s}}{\partial \rho^{i}}-\rho^{i} \frac{\partial A^{i}}{\partial \rho^{i}}-\rho^{f} \frac{\partial A^{f}}{\partial \rho^{i}}+\kappa\right] \dot{\rho}^{i}+} \\
& {\left[-\rho^{s} \frac{\partial A^{s}}{\partial \rho^{f}}-\rho^{i} \frac{\partial A^{i}}{\partial \rho^{f}}-\rho^{f} \frac{\partial A^{f}}{\partial \rho^{f}}\right] \dot{\rho}^{f}+} \\
& {\left[\vec{u} \rho^{i} \frac{\partial A^{i}}{\partial E^{s}}-\vec{u} \rho^{f} \frac{\partial A^{f}}{\partial E^{s}}\right] \cdot \vec{\nabla} E^{s}+} \\
& {\left[-\left(E \circ \frac{\rho^{s} \partial A^{s}+\rho^{f} \partial A^{f}+\rho^{i} \partial A^{i}}{\partial E^{s}} \circ E^{c}\right)+n^{s} \lambda L I+\underline{a}^{s}\right]: \vec{\nabla} \vec{v}^{s}+} \\
& {\left[\kappa \rho^{i} \underline{I I}+\underline{\sigma}^{i}\right]: \vec{\nabla} \vec{v}^{i}+\left[n^{f} \lambda \underline{I}+\underline{\rho}^{f}\right]: \vec{\nabla} \vec{v}^{f}+} \\
& \left(\rho^{s} \frac{\partial A^{s}}{\partial \rho^{f}} \vec{\nabla} \rho^{f}+\lambda \vec{\nabla} n^{f}\right) \circ \vec{u}- \\
& \left(\rho^{s} \frac{\partial A^{s}}{\partial \rho^{i}} \vec{\nabla} \rho^{i}+\vec{\pi}^{s}\right) \circ \vec{v}- \\
& \left(\vec{\pi}^{f}+\rho^{f} \frac{\partial A^{f}}{\partial \rho^{i}} \vec{\nabla}^{i}-\rho^{i} \frac{\partial A^{i}}{\partial \rho^{f}} \vec{\nabla} \rho^{f}\right) \circ \vec{\omega} \geq 0
\end{aligned}
$$

Insertion of the constitutive relations (3.22) into the extended entropy inequality (3.18) leads to an inequality that is for the first six terms linear in the clerivatives:

$$
\dot{\rho}^{i}, \dot{\rho}^{f}, \vec{\nabla} \vec{v}^{s}, \vec{\nabla} \vec{v} f, \vec{\nabla} \vec{v}^{i}, \vec{\nabla} E^{s}
$$

The inequality (3.25) must hold for all primary unknown fields: $\rho^{s}, \rho^{r}, \rho^{i}, \vec{v}^{s}, \vec{v}^{r}, \vec{v}^{i}$. It must also hold for arbitrary values of the above mentioned derivatives (3.26). By choosing arbitrary values for the derivatives one defines a path, either in space or time for the primary fields. To ensure that the inequality (3.25) holds for arbitrary values of the derivatives, the primary unknown fields should therefore comply with the condition that terms inside the square brackets of the first six terms vanish independently:

$$
\begin{aligned}
& \kappa=\rho^{s} \frac{\partial A^{s}}{\partial \rho^{i}}+\rho^{f} \frac{\partial A^{f}}{\partial \rho^{i}}+\rho^{\prime} \frac{\partial A^{i}}{\partial \rho^{i}} \\
& 0=\rho^{i} \frac{\partial A^{i}}{\partial \rho^{f}}+\rho^{\prime} \frac{\partial A^{f}}{\partial \rho^{f}}+\rho^{s} \frac{\partial A^{s}}{\partial \rho^{f}} \\
& \underline{0}=\vec{u} \rho^{i} \frac{\partial A^{i}}{\partial E^{s}}-\vec{u} \rho^{f} \frac{\partial A^{f}}{\partial E^{s}} \\
& \underline{a}^{i}=-\rho^{i} \kappa \underline{I I} \\
& \underline{a}^{s}=-\lambda n^{s} \underline{I I}+E \circ \frac{\rho^{s} \partial A^{s}+\rho^{f} \partial A^{f}+\rho^{i} \partial A^{f}}{\partial \mathbb{E}^{s}} \circ E^{c} \\
& \underline{a}^{\prime}=-\lambda n^{f} \underline{I I}
\end{aligned}
$$


leaving as inequality:

$$
\begin{aligned}
& \left(\rho^{s} \frac{\partial A^{s}}{\partial \rho^{f}} \vec{\nabla} \rho^{f}+\lambda \vec{\nabla} n^{f}\right) \circ \vec{u} \\
& -\left(\rho^{s} \frac{\partial A^{s}}{\partial \rho^{i}} \vec{\nabla} \rho^{i}+\vec{\pi}^{s}\right) \circ \vec{v} \\
& -\left(\vec{\pi}^{f}+\rho^{f} \frac{\partial A^{f}}{\partial \rho^{i}} \vec{\nabla} \rho^{i}-\rho^{i} \frac{\partial A^{l}}{\partial \rho^{f}} \vec{\nabla} \rho^{f}\right) \circ \vec{\omega} \geq 0
\end{aligned}
$$

By choosing:

$$
\begin{aligned}
B_{u} \cdot \vec{u} & =\rho^{s} \frac{\partial A^{s}}{\partial \rho^{f}} \vec{\nabla} \rho^{f}+\lambda \vec{\nabla} n^{f} \\
-\underline{B}_{w} \circ \overrightarrow{\mathrm{v}} & =\rho^{s} \frac{\partial A^{s}}{\partial \rho^{s}} \vec{\nabla} \rho^{i}+\vec{\pi}^{s} \\
-\underline{B}_{\omega} \circ \vec{\omega} & =\rho^{\prime} \frac{\partial A^{f}}{\partial \rho^{i}} \vec{\nabla} \rho^{i}-\rho^{i} \frac{\partial A^{i}}{\partial \rho^{f}} \vec{\nabla} \rho^{f}+\vec{\pi}^{f}
\end{aligned}
$$

and $\underline{B}_{y}, \underline{B}_{w}$ and $\underline{B}_{w}$ as semi-positive definite tensors inequality $(3.28)$ is always fulfilled. This choice may be too strict: the demand of the sum of the three components of $(3.28)$ not being smaller than zero is replaced by the requirement that each component itself is not smaller than zero. The introduced tensors are not independent because of the relation between the three relative velocities (3.21). However, we keep them separated for the ease of derivation in the subsequent paragraphs.

\subsubsection{Identification of Lagrange multipliers}

As stated before all physical properties are averaged values. The fluid is modelled macroscopically non-viscous. The constitutive relation for such a barotropic fluid is given by:

$$
\underline{\mathbf{E}}^{f}=-p \underline{I I}
$$

with $p$ the hydrodynamic pressure.

$\underline{\underline{E}}^{f}$ the microscopic Cauchy stress tensor of the fluid.

The Cauchy stress tensor of the fluid $g^{f}$, used in our constitutive theory is the bulk volume average of $\underline{\underline{\Sigma}}$ :

$$
\underline{Q}^{f}=\underline{\mathbf{\Sigma}}^{f}=\frac{1}{V_{e}} \int_{V_{t}^{\prime}} \Sigma^{f} d V=\frac{1}{V_{e}} \int_{V_{t}^{\prime}}-p \amalg I V
$$

The size of the elementary averaging volume $\mathrm{V}_{\mathrm{c}}$ is determined by the distribution of the constituents present within the volume. Intervertebral disc tissue is mainly a mixture on a molecular scale. Therefore the averaging volume is very small compared to a tissue sample. Within this volume it is permissible to assume that the hydrodynamic pressure is 
constant:

$$
\underline{a}^{t}=-\frac{V_{e}^{f}}{V_{e}^{f}} p L=-n t p L
$$

Comparing this result with the constitutive relationship found by making use of the entropy inequality (3.27.6) shows that the Lagrange multiplier $\lambda$ equals the hydrodynamic pressure p. The constitutive relationship for the Cauchy stress of the solid is also determined by $\lambda(3.27 .5)$. Deformation of the solid matrix is achieved either by the hydrodynamic pressure or the contact between the solid particles.

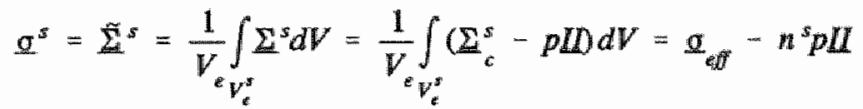

with $\quad \sum^{s}$ the microscopic Cauchy stress tensor of the solid

$\Sigma_{\mathrm{s}}$ the microscopic contact Cauchy stress tensor of the solid

$\sigma_{\text {eff }}$ the effective Cauchy stress

Comparing this result with the outcome of the entropy inequality $(3.27 .5)$ yields:

$$
\begin{gathered}
\underline{a}_{\text {eff }}=E \cdot \frac{\rho^{s} \partial A^{s}+p^{p} \partial A^{f}+\rho^{i} \partial A^{\prime}}{\partial E^{s}} \circ E^{c} \\
\lambda=p
\end{gathered}
$$

Again $\lambda$ equals the hydrodynamic pressure. The effective Cauchy stress is caused by the deformation of the wet solid matrix, in absence of the fluid phase. In physico-chemical theories (Bowen, 1980) it is common to define a chemical potential tensor by:

$$
\underline{K}^{\alpha *}=A^{\alpha} \underline{U}-\frac{\underline{\underline{Q}}^{\alpha}}{\rho^{\alpha}}
$$

Substituting the constitutive relationship for $\underline{q}^{i}(3.27 .4)$ and $\underline{q}^{f}(3.27 .6)$ gives:

$$
\begin{aligned}
& K^{i}=A^{i} U+\measuredangle I \\
& K^{f}=A^{f} U+\frac{p}{\Gamma^{\prime}} U
\end{aligned}
$$

Two conclusions may be drawn. It is obvious that the chemical potential tensors $\mathbb{K}^{i}$ and $\mathbb{K}^{\prime}$ are scallar functions: $\mu^{i}$ and $\mu^{i}$. The Lagrange multiplier $\kappa$ is the difference between the chemical potential and the Helmholtz free energy of the salt. Comparing this result with the chemical potential of the fluid, $\kappa$ may be interpreted as the pressure dependence of the chemical potential of the ions. It relates the volume change to a pressure change. For the range of pressures encountered in intervertebral disc tissue the value of $k$ is small compared to the value of the Hemholtz free energy $A^{j}$ and therefore neglected (Richards, 1980). This results in $\underline{Q}^{i} \approx 0$. Expressions for the Helmholtz free energy of a uniwalent salt as a function of the composition may be found in the physico-chemical literature (Richards, 1980): 


$$
\begin{aligned}
& A^{\prime}=A_{0}^{\prime}+\frac{R T}{M^{1}}\left[\ln \left(f^{*} x^{+}\right)+\ln \left(f^{\prime} x\right)\right]=A_{0}^{i}+\frac{R T}{M^{\prime}} \ln \left(f^{2} x^{+} x\right) \\
& A^{\prime}=A_{0}^{f}+\frac{R T}{M^{f}} \ln \left(f^{\prime} x^{\prime}\right)
\end{aligned}
$$

with $\mathrm{A}_{0}^{\mathrm{\alpha}}$ Helmholt free energy in the reference state

R universal gas constant

T absiolute temperature

$\mathrm{M}^{\infty}$ mole mass

$\mathrm{M}^{\mathrm{i}}=\mathrm{M}^{+}+\mathrm{M}^{-}$the molar mass of the neutral salt

$f^{*}$ activity coefficient

$f_{ \pm}^{2}=f^{*} f^{-}$the mean activity coefficient

$x^{e x}$ mole fraction

The activity coefficient is a function of the composition of the tissue. The mole fraction $x^{\text {ox }}$ is defined as the number of moles of constituent $\alpha\left(\mathrm{m}^{\alpha}\right)$, divided by the total number of moles in the tissue:

$$
x^{\alpha}=\frac{m n^{\alpha}}{\Sigma m^{\alpha}} \quad \Sigma x^{\alpha}=1
$$

The Helmholiz free energy of the ions is split into those of the anions and cations. The mole fraction of the anions does not equal the mole fraction of the cations because of the presence of ionized proteoglycans.

\subsubsection{The momentum interactions}

To complete the constitutive theory explicit closed expressions for the momentum interaction terms $\overrightarrow{\pi^{3}}, \overrightarrow{\pi^{f}}$ and $\overrightarrow{\pi^{i}}$ should be obtained. Substituting (3.27.2) in (3.29.3), substituting (3.29.1) in this result, and by making use of (3.21.3) gives an expression for $\overrightarrow{\pi^{r}}$ :

$$
\vec{\pi}^{f}=-\underline{B}_{\omega} \circ \vec{\omega}-\underline{B}_{4} \circ \vec{u}+p \vec{\nabla} n^{f}-p^{\prime} \vec{\nabla} A^{f}
$$

The momentum exchange of the fluid is caused by the relative flow of the ions with respect to the fluid, the relative flow of fluid with respect to the solid matrix (often called the Stokes drag), the buoyancy force as defined by Bowen (1980) and the gradient of the Helmholtz free energy. Starting with (3.11.4), substituting the sum of (3.29.2) and (3.29.3) and by making use of (3.27.1), yields an expression for $\overrightarrow{\pi^{i}}$ :

$$
\vec{\pi}^{i}=-\left(\vec{\pi}^{f}+\vec{\pi}^{s}\right)=\underline{B}_{u} \circ \overrightarrow{\mathrm{v}}+\underline{B}_{\omega} \circ \vec{\omega}+k \vec{\nabla} \rho^{i}-\rho^{i} \vec{\nabla} A^{i}
$$

The momentum exchange of the ions can be described in the same mathematical way as the momentum exchange of the fluid. An expression for $\bar{\pi}^{\mathrm{s}}$ can be obtained by combining (3.39) and (3.40).

Substituting the relationships found for the momentum exchange of the ions and fluid (3.39 and 3.40) in the respective balances of momentum (3.11.2 and 3.11.3) and making use of the constitutive relationships for $\underline{g}^{f}$ and $\underline{\sigma}^{3}(3.27 .4$ and 3.27.6) and the definition of the chemical potential $(3.36)$, yields: 


$$
\begin{aligned}
& -\rho^{i} \vec{\nabla} \mu^{i}+B_{\omega} \circ \vec{\omega}+\underline{B}_{\omega} \circ \vec{U}=\overrightarrow{0} \\
& -\rho / \bar{\nabla} \mu^{f}-\underline{B}_{\omega} \circ \vec{\omega}-\underline{B}_{u} \circ \vec{u}=\overrightarrow{0}
\end{aligned}
$$

Adding these two equations ( 3.41 ) yields:

$$
\underline{B}_{v}{ }_{v} \vec{v}-\underline{B}_{\mu} \circ \vec{u}=\rho^{i \vec{\nabla}} \mu^{i}+\rho / \vec{\nabla} \mu^{f}
$$

This relation may be viewed upon as an extended form of Darcy's law: relative fluid and ion flow is caused by the gradient of the chemical potential of the ions and fluid. Substituting the definition of the chemical potential (3.36) and the expression for the Helmholtz free energy (3.37) results into:

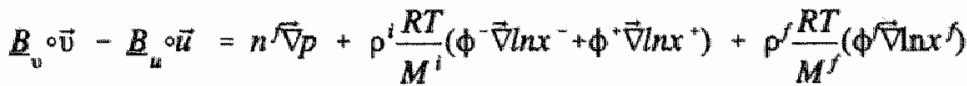

$$
\begin{aligned}
& \phi^{\alpha}=\left(1+\frac{\partial \ln f^{\alpha}}{\partial \ln x^{\alpha}}\right) \quad \alpha=+,-, f
\end{aligned}
$$

with $\phi^{\alpha}$ the osmotic coefficient

The mole fractions are rewritten to concentrations per unit fluid by:

$$
x^{\alpha}=\frac{m^{\alpha}}{\sum m^{\alpha}}=\frac{m^{\alpha} / V^{f}}{\sum m^{\alpha} / V^{f}}=\frac{c^{\alpha}}{\sum c^{\alpha}}
$$

Both in the in vivo situation and in all the experimental conditions which are considered in a following paragraph, the ion concentrations $\mathrm{c}^{+}$and $\mathrm{c}^{-}$are less than 1 mole/l. Because of the high molecular weight of both the proteoglycans and the collagen their concentrations, symbolised by $c^{s}$ can be neglected. The fluid concentration is approximatelly 55 mole/l. Hence, in a first approximation intervertebral disc tissue is a dilute solution:

$$
\begin{aligned}
& \left.\vec{\nabla}\left(\ln x^{f}\right)=\vec{\nabla} \ln \left(\frac{c^{f}}{c^{f}+c^{s}+c^{*}+c^{-}}\right)\right] \approx-\frac{\vec{\nabla}\left(c^{+}+c^{-}\right)}{c^{f}} \approx-\frac{M^{f}}{\Gamma^{f}} \vec{\nabla}\left(c^{+}+c^{-}\right) \\
& \left.\vec{\nabla}\left(\ln x^{*}\right)=\vec{\nabla} \ln \left(\frac{c^{*}}{c^{f}+c^{s}+c^{*}+c^{-}}\right)\right] \approx \frac{\vec{\nabla} c^{*}}{c^{*}} \\
& \vec{\nabla}\left(\ln x^{-}\right)=\vec{\nabla} \mid \ln \left(\frac{c^{-}}{c^{f}+c^{s}+c^{*}+c^{-}}\right] \approx \frac{\vec{\nabla} c^{-}}{c^{*}}
\end{aligned}
$$

Substitution of (3.45) in (3.43) and multiplying the result with $n^{f} \underline{B}_{u}^{-1}$ yields:

$$
\begin{aligned}
& n^{f} \vec{u}=n^{f} B_{u}^{-1} \circ B_{v} \circ \vec{v}-K^{\circ} \circ \vec{\nabla} p+K^{+} \circ \vec{\nabla} c^{*}+K^{\infty} \circ \ddot{\nabla} c^{-} \\
& \underline{K}=\left(n^{2}\right)^{2} B_{*}^{-1} \\
& \underline{K}^{\mathrm{m}}=\phi^{\prime} R T\left(1-\frac{c^{i} \phi^{\alpha}}{c^{\alpha} \phi^{\prime}}\right) K \quad \alpha=*_{*}-
\end{aligned}
$$

with $\underline{K}$ the hydraulic permeability tensor $\underline{\mathrm{K}}^{*}$ and $\underline{\mathrm{K}}^{-}$the ionic hydraulic permeability 
Relative motion is governed by the gradient in the hydraulic pressure, and the gradients in the anion and cation concentration. Each of these driving forces has its one permeability. In a first approximation it is assumed that:

$$
\begin{aligned}
& \|\vec{u}\|_{i} \quad\left\|B_{u}^{\omega 1} \circ B_{v} \circ \vec{v}\right\| \\
& K^{\alpha}=\phi^{\prime} R T\left(1-\frac{c^{i} \phi^{*}}{c^{\prime \prime} \phi^{t}}\right) K \approx \phi R T K
\end{aligned}
$$

with the total osmotic coefficient

No external electrical field is applied: charge neutrality is maintained at all time: the total concentration of negative ions equals the total concentration of the positive ions.

$$
c^{-}+c^{p s}=c^{*}
$$

with $c^{28}$ the fixed charged concentration due to the negatively ionized proteoglycans.

Following the results of equations (3.42) through (3.48) Darcy's law is expressed as:

$$
n^{f}\left(\vec{v}^{f} \vec{v}_{\vec{v}}\right)=-K \circ\left[\vec{\nabla} p-\phi R T\left(2 \vec{\nabla} c^{-}+\vec{\nabla} c^{p g}\right)\right]
$$

Relative fluid flow is governed by gradients of both a hydrodynamic pressure and an ion concentration. No fluid flow will occur when the pressure gradient is compensated by the ion-concentration gradient. In the absence of ions the classical form of Darcy's law is obtained.

\subsubsection{Equilibrium}

Consider a piece of tissue in equilibrium with an external solution of $\mathrm{NaCl}$. Because the tissue is in equilibrium, no material fluxes occur. Hence, the gradients of the chemical potential of both the ionic species and the fluid equal zero. The chemical potential of the lons within the tissue equals the value of the ions in the externall salt solution. Based on $(3.36 .1),(3.37 .1)$ and $k \approx 0$ this results for the ions (barred quantities refer to the external solution) into:

$$
f_{i}^{2} \bar{c}^{*} \bar{c}^{-*}=f_{t}^{2} c^{*} c^{-}
$$

In equilibrium the chemical potential of the fluid is also constant. Substituting (3.37.2) into $(3.36 .2)$ and linearizing the result like $(3.45 .1)$ yields for the fluid:

$$
\bar{p}-R T \bar{\phi}\left(\bar{c}^{*}+\bar{c}^{-}\right)=p-R T \phi\left(c^{+}+c^{-}\right)
$$

In the external solution charge neutrality is maintained at al time: the anion concentration equals the cation concentration:

$$
\bar{c}^{*}=\bar{c}^{-}=\bar{c}
$$

Of course charge neutrality is also maintained within the tissue (3.48). Combining the electro neutrality with $(3.50)$ yields the ion distribution within the tissue: 


$$
\begin{aligned}
2 c^{*} & =c^{p s}+\sqrt{\left(c^{P B}\right)^{2}+4 f^{2} \vec{c}^{2}} \\
2 c^{-} & =-c^{P E}+\sqrt{\left(c^{P g}\right)^{2}+4 f^{2} \vec{c}^{2}} \\
f^{2} & =\frac{f_{*}^{2}}{f^{2}}
\end{aligned}
$$

with $\mathrm{f}^{2}$ the mean activity coefficient quotient

This distribution resembles the Donnan ion distribution given in chapter 2 . The quotient $\mathrm{f}^{2}$ representing the deviation from ideality. Without loss of generality the hydrodynamic pressure in the external solution is set to zero. Using the ion distribution $(3.53)$ the hydrodynamic pressure in the tissue is given by:

$$
p=R T\left\{\phi \sqrt{\left(c^{p g}\right)^{2}+4 f^{2} \bar{c}^{2}}-2 \bar{\phi} \bar{c}\right\}
$$

Again this can be interpreted as the non-ideal Donnan osmotic pressure. In experimental set-ups often such solution tissue interface exist. Even in vivo they exist. For instance in the intervertebral disc: the endplate vertebral body interface and the outer anulus. As stated in the introduction the readjustment of the local electrical fields takes nano seconds. Compared to the experimental time, this time lag is negligible. The interface is always in equilibrium with the external solution: (3.53) and (3.54) are used as boundary conditions. However, due to deformations in the tissue the local PG concentration changes. The equilibrium salt concentration and hydrodynamic pressure in the tissue changes according to the change of both the proteoglycan concentration and the external salt concentration at the interface. The boundary conditions change in time, even when the external salt concentration is constant.

\subsubsection{Diffusion equation of the ions}

In order to find the diffusion equation for the ions the ion velocity $\vec{w}^{i}$ in the mass balance (3.7.3) can be eliminated by using the modified balance of momentum $(3.41 .1)$, resulting in the diffusion equation:

$$
\begin{aligned}
& \left.\frac{\partial \rho^{i}}{\partial t}=\vec{\nabla} \circ\left(\underline{D}_{\mu} \cdot \vec{\nabla} \mu^{i}-\rho^{t} \underline{D}_{\omega}{ }^{a} \vec{v}^{f}-\rho^{t} \underline{D}_{v} \vec{v}^{s}\right)\right\} \\
& D_{\mu}=\left(\rho^{t}\right)^{2}\left(B_{\omega}+B_{v}\right)^{-1} \\
& D_{\omega}=\left(B_{\omega}+E_{v}\right)^{-1} \circ B_{\omega} \\
& D_{v}=\left(B_{\omega}+\underline{B}_{v}\right)^{-1}{ }^{-1} \underline{B}_{v}
\end{aligned}
$$

The total ion flux includes diffusion caused by the gradient of the chemical potential and convection by the macroscopic fluid and solid flow. Using the ion mass density per unit fluid volume $\left(Q^{i}\right)$ instead of the mass density per unit tissue volume $\left(\rho^{i}\right)$, and assuming that the convection by the solid is negligible compared to the convection by the fluid, and substituting the mass balance of the fluid into this result, yields: 


$$
\begin{aligned}
& \left\|\vec{v}^{f}\right\|>\left\|\left(\boldsymbol{R}_{\omega}^{-1} \circ \boldsymbol{B}_{v}\right) \circ \vec{v}^{s}\right\| \\
& \left(B_{w}+\underline{B}_{v}\right)^{-1} \circ B_{w}=I \\
& n^{j}\left(\frac{\partial e^{i}}{\partial t}+\vec{\nabla}_{Q} \omega_{v} f\right)=\vec{\nabla} \circ\left(D_{\psi} \circ \vec{\nabla}_{\mu}\right)
\end{aligned}
$$

In a similar way as used by the derivation of Darcy's law the gradient of the chemical poteritial can be expressed in terms of the ion concentrations per unit fluid volume. Through (3.6) these concentrations are related to the ion mass density per unit fluid. The cation concentration is eliminated using the charge neutrality (3.48). The gradient of the chemical potential is expressed in terms of the ion mass density per unit fluid volume and the proteoglycan concentration per unit fluid volume:

$$
\vec{\nabla} \mu^{i}=\frac{R T}{M^{i}}\left\{\left(\frac{\Phi^{-}}{c^{-}}+\frac{\Phi^{+}}{c^{+}}\right) \frac{\vec{\nabla} Q^{i}}{M^{i}}+\left(\frac{\Phi^{+}}{c^{+}}-\frac{M^{+}}{M^{i}}\right) \vec{\nabla} c^{p .8}\right\}
$$

Substituting this result into (3.56) and using (3.2) for the material time derivative, yields the diffusion equation for the mobile ions:

$$
\begin{gathered}
n^{f}\left(\dot{\mathrm{Q}}^{i}\right)_{f}-\vec{\nabla} \circ\left(D^{\prime} \circ \vec{\nabla} \mathrm{Q}^{+}\right)=\vec{\nabla} \circ\left(D^{p g} \circ \vec{\nabla} c^{P 8}\right) \\
D=\frac{1}{\left(M^{2}\right)^{2}}\left(\frac{\phi^{-}}{c^{-}}+\frac{\phi^{+}}{c^{+}}\right) D_{\mu} \\
D^{p g}=\frac{1}{M^{i}}\left(\frac{\phi^{+}}{c^{+}}-\frac{M^{+}}{M^{*}}\right) D_{\mu}
\end{gathered}
$$

with $\underline{D}$ the diffusion tensor of the ions

$\underline{D}^{\text {pg }}$ the diffusion tensor of the proteoglycans

\subsection{Summary of equations}

In this section the basic equations are summarized and rewritten in the form that is used in the numerical solution strategy. As we study isothermal conditions, the balance of energy is omitted.

ninass balance of the mixture:

$$
\vec{\nabla} \circ \ddot{v}^{s}+\vec{\nabla} \circ\left(n^{f}\left(\vec{v}^{f}-\vec{v}^{s}\right)\right)=0
$$

mass balance of the ions:

$$
n^{f}\left(\dot{Q}^{i}\right)_{f}-\vec{\nabla} \circ\left(\underline{D} \circ \vec{\nabla}_{Q}{ }^{i}\right)=\vec{\nabla} \circ\left(D^{p g} \circ \vec{\nabla}_{C}{ }^{p g}\right)
$$

ballance of momentum of the mixture:

$$
\vec{\nabla} \circ \underline{\underline{Q}}^{s}+\vec{\nabla} \circ \underline{\underline{Q}}^{f}+\vec{\nabla} \circ \underline{\underline{Q}} \underline{a}^{i}=\overrightarrow{0}
$$

constitutive relations: 


$$
\begin{aligned}
& \underline{\underline{a}}^{i}=-\rho^{i} \mathbf{K} I I=\underline{0} \\
& \underline{\sigma}^{s}=-p n^{s} \underline{I}+\underline{\sigma}_{\text {tff }} \quad \underline{\sigma}_{\text {eff }}=E \cdot \frac{\rho^{s} \partial A^{s}+p^{f} \partial A^{f}+p^{i} \partial A^{i}}{\partial E^{s}} \cdot E^{c} \\
& \underline{\sigma}^{f}=-p n^{f} I I
\end{aligned}
$$

Assuming the osmotic coefficient $\phi$ independent of the spatial coordinates, Darcy's law is rewritten to:

with $\quad \tilde{\omega}$ the osmotic retention

$$
\begin{array}{ll}
n^{f}\left(\vec{v}^{f}-\vec{v}^{s}\right) & =-K \circ \vec{\nabla}(p-\tilde{\omega}) \\
\tilde{\omega} & =\phi R T\left(2 c^{-}+c^{p g}\right)
\end{array}
$$

The ionized proteoglycans are the origin of the fixed charge concentration. As the proteoglycans are entangled in the collagen network the concentration varies with the deformation. Both the solid and the fluid are intrinsically incompressible: a volume change is only achieved by expelling fluid out of the tissue.
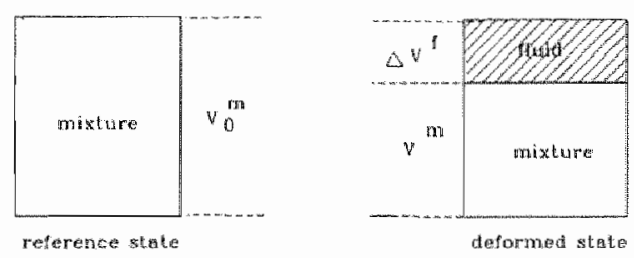

figure 3.1 The tissue deformation. A volume change is only achieved by expelling fluid out of the tissue.

The fixed charge concentration in the deformed state is expressed as:

$$
c^{p s}=\frac{\text { total amount } P G}{V^{f}}=\frac{c_{0}^{p g} V_{0}^{f}}{V_{0}^{f}-(1-J) V_{0}^{m}}=\frac{c_{0}^{p g}}{1-\frac{(1-J)}{n_{0}^{f}}}
$$

with $\quad c_{g}^{p g}$ the fixed charge concentration in the reference state

$\mathrm{n}_{0}^{\prime}$ the porosity in the reference state

$\mathrm{J}$ the volume change

Substituting the constitutive relations (3.27.4-6) in the balance of momentum of the mixture yields:

$$
\vec{\nabla} \circ\left(\underline{\alpha}_{\text {eff }}-p \underline{I I}\right)=\overrightarrow{0}
$$

Substituting Darcy's law (3.59) in the mass balance of the mixture (3.8) yields: 


$$
\vec{\nabla} \circ \vec{v}^{s}-\vec{\nabla} \cdot[K \cdot \vec{\nabla}(p-w)]=0
$$

In the numerical solution method it is convenient to use the material time derivative which follows the solid matrix. In a first approximation the dot product of $\underline{D}^{-1}$ and $\underline{D}^{\text {pe equals the }}$ unity matrix multiplied by a constant scalar $\xi$. Thus, using (3.3) the mass balance of the ions $(3.58)$ is rewritten to:

$$
\left(\mathrm{e}^{\dot{b}}\right)_{s}+\vec{\nabla}_{\mathrm{e}}^{l} \circ\left(\vec{v}^{f}-\vec{v}^{s}\right)=\frac{1}{n^{f}} \vec{\nabla} \cdot\left[D \circ\left(\vec{\nabla} \mathrm{e}^{i}+\xi \vec{\nabla} c c^{p g}\right)\right]
$$

with $\xi=\underline{D}^{-1} \circ \underline{D}^{p t}=\xi \underline{I I}$

Using the same derivation as for $\mathrm{c}^{\mathrm{pg}}$, the porosity in the deformed state is given by:

$$
n^{f}=1-\frac{1-n_{0}^{f}}{J}
$$

The equations (3.59) until (3.64) form the set of equations describing the triphasic swelling behaviour of intervertebral disc tissue. The initial conditions and the boundary conditions are given by the equilibrium results:

$$
\begin{aligned}
& 2 c^{-}=-c^{p g}+\sqrt{\left(c^{p g}\right)^{2}+4 f^{2} \overline{c^{2}}} \\
& p=R T\left\{\phi \sqrt{\left(c^{p g}\right)^{2}+4 f^{2} \bar{c}^{2}}-2 \bar{\phi} \bar{c}\right\}
\end{aligned}
$$

The boundary condition for the momentum of the mixture is:

$$
\left(\sigma_{\text {eff }}-p D\right) \circ \vec{n}=\vec{t}
$$

with $\vec{t}$ the boundary stress vector

$$
n \text { the unit normal vector on the boundary }
$$

In the triphasic theory presented so far the chemo-mechanical behaviour of IVD tissue is governed by three coupled partial differential equations. The necessary initial and boundary conditions are also obtained. Based on the entropy principle constitutive restrictions are derived. Unfortunately only restrictions are found and no closed expressions. On a purely theoretical basis this is the maximum result which can be achieved for a macro-continuum theory. To gain more insight in the behaviour of the tissue, experiments are vital. In order to complete the set of equations closed expressions are needed for:

-the mean activity coefficient quotient $f^{2}$

-the osmotic coefficient $\phi$

-the diffusion tensor $\underline{D}$

-the permeability tensor $\underline{\mathbb{K}}$

-the stress-strain relation

Also the initial tissue composition should be known:

-the proteoglycan concentration cos

-the volume fraction of the fluid $\mathrm{n}_{0}^{\mathrm{f}}$

A part of this information can be found in the literature. The material behaviour obtained from experimental data is only valid within the theoretical framework used for the 
analysis. Special care should be taken by the interpretation of experimental results. For instance, if the aggregate modulus is determined using a biphasic theory the value is useless for a triphasic formulation. Especially if large deformations occur, resulting in osmotic effects, which are accounted for in a biphasic formulation through the aggregate modulus (section 3.5)

The initial fixed charge density profiles and the initial water content profiles in the IVD have been measured by Urban and Maroudas (1979) and by Jolnnstone et al. (1992). In chapter 2 (section 2.5.1) the total amount of water is divided between two compartments: the intra- and extra-fibrilar water. The amount of intra-fibrilar water is almost constant, independent of the deformation (Maroudas and Barnon, 1981). Recent experimental results on articular cartilage however show a decrease of intra-fibrilar water with increasing pressure (Maroudas et al., 1991). The change of intra-fibrilar water levels off for the pressure range encountered in vivo and during our experiments (chapter 5). If the low collagen content of the nucleus is also taken into account it seems reasonable to assume a constant amount of intra-fibrilar water in the nucleus. For the anulus it is assumed that in a first approximation the content of intra-fibrilar water is constant. Hence, the amount of water expelled from the tissue originates from the extra-fibrilar compartment. In a first approximation the proteoglycan concentration and the water content are based on the extra-fibrilar water.

Maroudas (1980) and Urban and Maroudas (1979) conclude that the biophysical properties of IVD tissue are comparable with those of articular cartilage. The mean activity coefficient of cartilage from the femoral head is at most $10 \%$ lower than the one of the external solution of $0.15 \mathrm{M} \mathrm{NaCl}$. Usually the deviation is less than $5 \%$. Hence, the mean activity coefficient quotient $f^{2}$ can be approximated by 1.05 . By the same authors the osmotic coefficient for cartilage is determined as 0.8 times the value of the external $0.15 \mathrm{M} \mathrm{NaCl}$ solution. The latter is found in text books concerning electrolyte solutions (Robinson and Stokes, 1970).

Maroudas (1975, 1980) reports values for the diffusion coefficients of $\mathrm{Na}^{+}$and $\mathrm{Cl}^{-}$. The values are $40 \%$ of those in an aqueous solution of equiwalent ionic strength (table 3.1).

\begin{tabular}{|l|l|l|}
\hline species & $\begin{array}{l}\text { Diffusion coefficient } \mathrm{D} \\
\text { in cartilage } 10^{-10} \mathrm{~m}^{2} \mathrm{~s}^{-1}\end{array}$ & D/D \\
\hline chloriti \\
\hline sodium $\mathrm{Cl}^{+}$ & $7.5-8.5$ & $0.40-0.45$ \\
\hline $\mathrm{NaCl}$ & $4.8-5.5$ & 0.40 \\
\hline
\end{tabular}

table 3.1 Diffusion coefficients in cartilage (Maroudas, 1975)

The diffusion coefficients of the different ionic species have to be combined in order to obtain the diffusion coefficient of $\mathrm{NaCl}$. The hamonic average diffusion coefficient is given by (Cussler, 1986): 


$$
D_{\text {WaCl }}=\frac{2}{\frac{1}{D_{C l^{*}}}+\frac{1}{D_{\text {Nal }^{*}}}}
$$

The diffusion is dominated by the slower ion. However, there is only one diffusion coefficient for the two diffusing ions because the ions are electrostatically coupled.

All material quantities discussed above are obtained in approximately the same theoretical framework as the one presented in this chapter. The values found in the literature are suitable. However, this is not true for the values found for the permeability and the stress-strain relation. As discussed in chapter 2 (section 2.5) no uniform results are obtained by different investigators using different swelling theories. More uniform results are obtained if the non-swelling biphasic theory is used. However, both the triphasic intrinsic stiffness and osmotic effects contribute to the biphasic apparent stiffness of the tissue. Most biphasic results are also limited to the case of small deformations. In that case an estimation, based on literature values, for the triphasic stiffness can be made as will be illustrated in section 3.5. First the permeability and the stress-strain relation will be discussed in general and biphasic terms.

The stress strain relation (3.27.5) is rewritten by introducing the effective PiolaKirchhoff stress, related to the effective Cauchy stress by:

$$
\begin{gathered}
\underline{S}_{e f f}=J E^{-1} \underline{o g}_{e f f f^{\circ}} E^{-c} \\
\underline{S}_{e f f}=p_{0}^{s} \frac{\partial A}{\partial \underline{E}}
\end{gathered}
$$

The Helmholtz free energy A is still a function of the Green-Lagrange strain, the fluid and ion mass density. The later two are replaced by one variable: the ion concentration per unit fluid. Initiated by Fung (1973), many expressions for A have experimentally been verified for different soft tissues:

-skin: Lanir (1979), Tong and Fung (1976), Oomens (1985)

-passive myocardium: Yin et al. (1987), Humphrey et al . (1990), Bovendeerd (1990)

-arterial wall: Demiray and Vito (1976), Takamizawa and Hayashi (1987)

-cartilage: Kwan et al. (1990)

Usually the resulting expression for $S_{\mathrm{taf}}$ is a combination of a linear, exponential or logarithmic function in the deformation terms. All expressions, except the one by Kwan (1990), have one disadvantage in common: if the displacement is increased during uniaxial compression the load needed increases to a maximum and thereafter decreases to zero. Lai et al. (1991) expand within their triphasic theory the Helmholtz free energy into a polynomial of the first and second invariants of the infinitesimal deformation tensor. The coefficients of the expansion depend on the ion concentration. Hence, the resulting elastic constants depend on the ion concentration.

The permeability is a function of tissue composition. Using a biphasic approach the permeability of several soft tissues has been determined:

-skin: Oomens (1985)

-cartilage: Mow et al. (1980)

-intervertebral disc: Urban and Maroudas (1980), Best et al. (1989), Guilak et al. (1990)

The first three authors report a strain dependent permeability: it decreases exponentially 
with the volume change. No dependency on the ion concentration is reported. For the intervertebral disc permeability values of 0.22 to $0.4010^{-15} \mathrm{~m}^{4} / \mathrm{Ns}$ are reported.

\subsection{One dimensional swelling}

In a confined swelling test a sample of tissue fits tightly in an impervious cylindrical chamber. A mechanical load is applied through a porous filter connected to a metal piston. The permeability of the filter is high compared to the permeability of the sample. Chemical loading is achieved by flowing a salt-water mixture through the filter. Consider a homogenous sample of intervertebral disc tissue in equilibrium with a hypertonic solution ( $\mathrm{NaCl}$ concentration $>1.5 \mathrm{M}$ ) and without a mechanical load. In this case the osmotic pressure is near zero and the sample is stress free. Suddenly the ionic strength of the bathing solution is lowered and a mechanical load is applied. After some time a new equilibrium is reached. In equilibrium the applied mechanical load $\mathrm{p}_{\mathrm{app}}$ is balanced by the osmotic pressure and by the forces due to the deformation of the solid matrix. The situation is considered in which the osmotic pressure is partially balanced by the applied load, so swelling will occur. For the ions and the fluid the equilibrium situation is discussed in section 3.3.3. The anion concentration is given by (3.53). The fluid pressure equals the non-ideal osmotic pressure (3.54). The proteoglycan concentration is given by (3.60). Because the specimen is lateraily confined the volume change is proportional to the height change. The fact that $\mathrm{H}_{\mathrm{A}}$ may depend on the local salt concentration (3.67) is ignored, resulting in a linear relation between the second Piola-Kirclhhoff stress and the Green-Lagrange strain. The equilibrium situation is given by:

$$
\begin{aligned}
& c^{p g}=\frac{c_{0}^{p g}}{1-\frac{1-\lambda}{n_{0}^{f}}} \\
& 2 c^{-}=-c^{p g}+\sqrt{\left(c^{p 8}\right)^{2}+4 f^{2} c^{2}} \\
& p \quad=\phi R T\left[\left(2 c^{-}+c^{p g}\right)-2 c\right] \\
& p_{a p p}=0.5 H_{A}\left(\lambda^{2}-1\right) \lambda-p
\end{aligned}
$$

with $\lambda$ the axial stretch, the quotient of actual and the reference height $\mathrm{H}_{\mathrm{A}}$ the aggregate modulus

As discussed in chapter 2 (section 2.5.1) both Maroudas and Urban with coworkers use (3.68.4) to characterise the swelling behaviour of cartilaginous tissues. The applied pressure as a function of the axial stretch for literature values: $H_{A}=0.25[\mathrm{MPa}], \mathrm{f}^{2}=1.05$ $[-], \phi=0.83[-], T=300[\mathrm{~K}], \mathrm{R}=8.314\left[\mathrm{JK}^{-1} \mathrm{~mol}^{-1}\right], \mathrm{ckg}_{0}^{\mathrm{B}}=0.310^{3} \mathrm{eq} \mathrm{m}^{-3}, \mathrm{n}_{0}^{f}=0.8[-]$ is given in fig. 3.2 . 


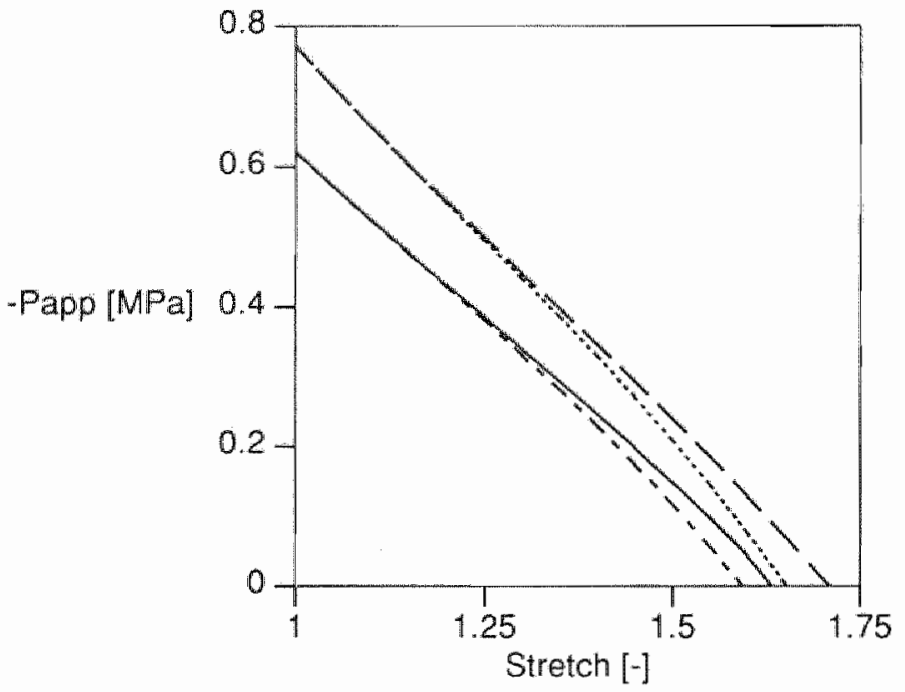

figure 3.2 Equilibrium results: applied pressure versus axial stretch during confined compression from a hypertonic reference state due to a change in the ionic environment ( $T_{6}$ the chemical expansion stress).

$\begin{array}{llll}\text { 1dash: } \mathrm{H}_{\mathrm{A}}=0.25 & {[\mathrm{MPa} ;} & \mathrm{T}_{\mathrm{c}}=0.5 \mathrm{c}^{\mathrm{pg}} & {[\mathrm{MPa}]} \\ \text { dot: } \mathrm{H}_{\mathrm{A}}=0.25 \exp \left(-0.1\left(\lambda^{2}-1\right)\right) & {[\mathrm{MPa} ;} & \mathrm{T}_{\mathrm{c}}=0.5 \mathrm{c}^{\mathrm{pg}} & {[\mathrm{MPa}]} \\ \text { solid: } \mathrm{H}_{\mathrm{A}}=0.25 & {[\mathrm{MPa} ;} & \mathrm{T}_{\mathrm{c}}=0.0 & {[\mathrm{MPa}]} \\ \text { sdash: } \mathrm{H}_{\mathrm{A}}=0.25 \exp \left(-0.1\left(\lambda^{2}-1\right)\right) & {[\mathrm{MPa}] ;} & \mathrm{T}_{\mathrm{c}}=0.0 & {[\mathrm{MPa}]}\end{array}$

The intercept with the horizontal axis is the free swelling stretch. The intercept with the vertical axis gives the pressure which is needed to keep the specimen at the reference height. Note this pressure is independent of the aggregate modulus, it is determined by the ionic composition of the tissue. Urban and Maroudas (1980) and Urban and McMullin $(1985,1988)$ have measured the applied pressure as a function of hydration $\left(\mathrm{g} \mathrm{H}_{2} \mathrm{O} / \mathrm{g}\right.$ dry weight). Hydration is almost linearly related to the axial stretch. Instead of the slightly convex curves of fig. 3,2 they have found concave curves. The concave tendency is pronounced for high, non physiological hydrations. In the physiological range the curves are slightly concave. The convexity of the results of 3.68 increases if a non-linear stiffening stress strain relationship is used. Such a stiffening could be caused by a deformation dependent stress-strain relationship (Holmes, 1986 and Kwan, 1990; both for cartilage), salt dependent elastic constants (Mow and Schoonbeck, 1984 and Eisenberg and Grodzinsky, 1985; both for cartilage) or a combination of both effects. As discussed in section 2.5 Lai et al. (1991) increased (for the case studied here) the swelling tendency with a so-called chemical expansion stress. In equilibrium while in contact with deionized water, the chemical expansion stress varies linear with the proteoglycan concentration. This results in an increase of the convexity of 3.68 . So using physically non-linear relation does not result into a shift from a convex curve into a concave curve (fig 3.2).

Many researchers use the non-steady state response to a confined compression test to measure the material properties of soft biological tissues:

-cartilage Mow et al. (1980) 
-skin and subcutis Oomens (1985)

-intervertebral disc Urban and Maroudas (1980), Best et al. (1989)

The tissue is (isometrically) equilibrated against an external solution, usually physiological saline. After equilibrium is reached a mechanical load is applied, while the external solution is unchanged. To analyze the transient behaviour some assumptions are useful to minimize the mathematical problems:

-the small strain theory is valid.

-because the reference situation is an equilibrium state and the external salt concentration does not change, the internal salt concentration is assumed at Donnan equilibrium at all time.

Combining the mass balance (3.62) and the balance of momentum (3.61) together with at linear stress-strain relationship yields for the displacement of this one-dimensional problem:

$$
\frac{\partial u}{\partial t}=\boldsymbol{K} \frac{\partial}{\partial x}\left\{H_{A} \frac{\partial u}{\partial x}-\tilde{\omega}\right\}
$$

The osmotic pressure $\bar{\omega}$ is a function of both the local salt and PG concentration. The salt concentration is approximated by the Donnan equilibrium (3.53). The proteoglycan concentration can be calculated by (3.60). The linearized osmotic pressure yields:

$$
\begin{aligned}
& \tilde{\omega} \approx \phi R T c_{0}^{p g} \sqrt{a}\left(1-\frac{1}{a n_{0}^{\prime}} \frac{\partial u}{\partial x}\right) \\
& a=1+4 f^{2}\left(\frac{\bar{c}}{c_{0}^{p g}}\right)^{2}
\end{aligned}
$$

Substitution of the latter in (3.69) results into the differential equation describing the mechanical response:

$$
\begin{aligned}
& \frac{\partial u}{\partial t}=K \frac{\partial}{\partial x}\left(H_{A}^{b i} \frac{\partial u}{\partial x}\right) \\
& H_{a}^{b i}=H_{A}+\frac{\phi R T c_{0}^{p g}}{n_{0}^{f} \sqrt{a}}
\end{aligned}
$$

with $H_{A}^{\text {bi }}$ the apparent biphasic stiffness

This equation looks the same as the one obtained by the pure biphasic theory. This is why fitting the experimental data to a biphasic theory gives such good results (Mow et al., 1980 and Oomens, 1987). Compared to the biphasic theory the apparent stiffness of the tissue is composed of two parts: the intrinsic stiffness of the solid matrix represented by $\mathrm{H}_{\mathrm{A}}$ and a osmotic contribution. By considering only the proteoglycan concentration effect and by neglecting the ionic contrubution Lanir (1987ab) found a comparable result. The intrinsic stiffness is independent of the salt concentration and represents the stiffness in an excess of salt. By decreasing the salt concentration the osmotic contribution increases, reaching a maximum in deionized water. In fig. 3.3 the normalised osmotic stiffness, defined as: 


$$
H_{\text {morm }}=\frac{H_{A}^{b i}(c)-H_{A}}{H_{A}^{b i}(c=0)-H_{A}}
$$

is compared with experimental results. The normalised osmotic stiffness calculated by (3.71) using a initial fixed charge density of 0.1 eq/l shows a good similarity with the results of Mow et al. (1984). Lai et al. (1991) use the values of Mow et al.. (1984) for their triphasic salt dependent stiffness using also an initial fixed charge density of 0.1 eq/l. The experimental results of Ejsenberg et al (1987) shows the same tendency but for salt concentrations above $0.2 \mathrm{M}$ the results deviate. Based on these data Eisenberg and Grodzinsky $(1985,1987)$ have used in their thermo-elastic analogue for the swelling behaviour of articular cartilage a salt dependent stress strain relation. According to them the salt dependent stiffness originates from the shielding of the charged proteoglycans and is associated with the solid component.

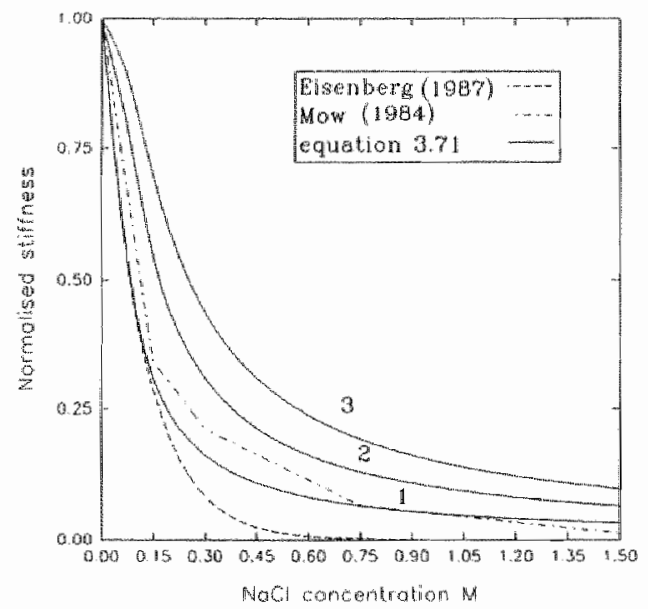

figure 3.3 Apparent normalised stiffness versus the $\mathrm{NaCl}$ concentration of the external bath. The curves based on equation 3.71 (solid lines) are for different proteoglycan concentrations:

1. $c_{g}^{p g}=0.1 \mathrm{eq} / 1,2 . c_{g}^{\mathrm{gg}}=0.2 \mathrm{eq} / 1,3 . c_{0}^{\mathrm{gg}}=0.3 \mathrm{eq} / 1$.

In the theory presented in this thesis the apparent stiffness originates from both the osmotic pressure, which is related to the fluid and ionic components and, the intrinsic stiffness of the solid matrix. This approach resembles the one of Urban and Maroudas (1980). They define the apparent stiffness as the slope of the swelling pressure versus hydration. The swelling pressure is defined as the difference between the osmotic pressure and the elastic tension in the collagen network. In physiological saline $(c=0.15 \mathrm{M})$, a reference $P G$ concentration of $0.15 \mathrm{eq} / \mathrm{l}$ and an osmotic coefficient of 0.830 the osmotic part is $0.139 \mathrm{MPa}$. Compared to the biphasic stiffness of the nucleus: $0.38 \mathrm{MPa}$ (Best, 1989), this contribution is not negligible. 


\subsection{References}

Atkin, R.J., Craine, R.E. Continum theories of mixtures: Basic theory and historical development. Quart. J. Mech. Appl. Math., 29:209-243, 1976.

Best, B.A., Guilak, F., Weidenbaum, M., Mow, V.C. Compressive stiffness and permeability of intervertebral disc tissues: variation with radial position, region and level. in: Advances in Bioenginering, BED Wol 15, p73-74, 1989.

Bowen, R.M. Theory of mixtures. in: Continuum Physics., (ed. Eringen, A.C.), Acad. Press, 1973.

Bowen, R.M. Incompressible porous media models by use of the theory of mixtures. Int. I. Eng. Sci., 18:1129-1148, 1980.

Cussler, E.L. Diffusion, mass transfer in fluid systems. Cambridge University Press, Cambridge, 1986.

Eisenberg, S.R., Grodzinsky, A.J. Swelling of articular cartilage and other connective tissues: electromechanochemical forces. J. Orthop. Res., 3:148-159, 1985.

Grodzinsky, A.I., Roth, V., Meyers, E., Grossman, W.D., Mow, V.C. The significance of electronechanical and osmotic forces in the nonequilibriun swelling behaviour of articular cartilage in tension. J. Biomech. Eng., 103:221-231, 1981 .

Hassanizadleh, M., Gray, W.G. General conservation quotations for multi phase systems: 1 Average procedure. Adv. Water Resources 2:131-??\%, 1979.

Holmes, M.H., Lai, W.M., Mow, V.C. Singular pertubation analysis of the nonlinear, flow-dependent compressive stress relaxation behaviour of articular cartilage. ASME J. Biomech. Eng., 107:206-218, 1985.

Johnstone, B., Urban, J.P.G., Roberts, S., Menage, J. The fluid content of the human intervertebral disc. Spine, 17:412-416, 1992.

Lai, W.M., Hou, J.S., Mow, V.C. A triphasic for the swelling and deformation behaviours of articular cartilage. J. Biomech. Engrg., 113:245-258, 1991.

Lanir, Y. Biorheology and fluid flux in swelling tissues. I Bicomponent theory for small deformations, including concentration effects. Biorheology. 24:173-1.87, 1987a.

Lanir, Y. Biorheology and fluid flux in swelling tissues. II Analysis of unconfined compressive response of transwersely isotropic cartilage disc. Biorheology, 24:189-205, 1987b.

Maroudas, A. Biophysical chemistry of cartilaginous tissues with special reference to solute and fluid transport. Biorheology, 12:233-248, 1975.

Maroudas, A. Physical chemistry of articular cartilage and the intervertebral disc. in : The joints and synovial fluid. Vol 2, (ed. Sokoloff. L.), Academic Press, New York, 1980.

Maroudas, A., Bannon, C. Measurement of swelling pressure in cartilage and comparison with the osmotic pressure of constituent proteoglycans. Biorheology, 18:619-632, 1981 .

Mow, V.C., Kuei, S.C., Lai, W.M. Biphasic creep and stress relaxation of articular cartilage in compression. J. Biomech. Engng., 102:73-84, 1980. 
Mow, V.C., Sehowbusk, J.M. Contribution of Donnan ownotic pressure towards the biphasic compressite modulue of aricular cantitage. Tratris. Orthop. Res. Soc, 9:262, 1984.

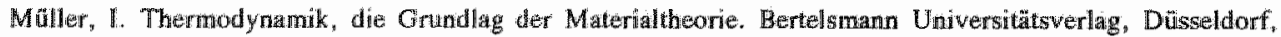
1973 .

Muller, I. Thermodynamics. Pimman Advanced Publishing Program, Boston, 1-202, 1985.

Oomens, C.W.J., Campen, D.H. van, Grootenboer, H.J., Boer, L.J. de, Experimental and theoretical compression studties on porcine skin. in: Biomechanics current intendisciplinary research. (Ed. Perren, S.M., Sehneider, E. $227 \times 232,1985$.

Oomena, C.W.J., Campen, D.H. van, Grootenboer, H.I. A mixture approach to the mechanics of skin. J. Homech. 20:877-885, 1987 .

Richards; E. O. An introduction to physical properties of large molecules in solution. Cambridge University Press, Cambridge, 1980 .

Robinson, R.A., Stokes; R.H. Eltectrolyte solwtions, Butterworth \& Co, London, 1970.

Slattery, J.C. Momentum, energy and mass transfer in continua. McGraw-Hill., New York, 1972.

Urban, 1.P.G., Maroudas, A. The measurement of fixed charge density in the interwertebral disc. Biochim. Biophys. Acta, 586:166-178, 1979 .

Urban, J., Marowdas, A. Measurement of swelling pressure and fluid fllow in the interwertebral disc with rafference to creep. Proc. Inst. Mech. Eng., C132/80:63-69, 1980.

Urbam, J.P.G., McMullin, J.F. Swelling pressure of the intervertebral dise: influence of proteoglycan and collagen contents. Biorheology, 22:145-157, 1985.

Urban, J.P.G., McMullin, J.F. Swelling pressure of the lumbar intervertebral discs: influence of age, spinal level, composition and degeneration. Spine, 13:179.187, 1988.

Whitaker, S. Advances in the theory of fluid motion in porous media. Ind. Engng. Chem. 61.14, 1969. 
The equations describing the mechanical behaviour of intervertebral disc tissue are three coupled partial differential equations in which geometric and physical non-linearities occur. To solve them for an arbitrary geometry and arbitrary boundary conditions we use the Finite Element Method. The differential equations are rewritten in an integral form by means of the weighted residual method, introducing weighting functions. By applying Gauss" theorem and rewriting with respect to the reference state (total Lagrange), nonlinear equations are obtained. These are linearized by means of the Newton-Raphson technique (section 4.1). In order to get a set of linear equations the weighted residual equations are discretized. The shape functions are chosen as weighting functions (Garlerkin method). This discretization results in a non-symmetric stiffness matrix (section 4.2). In section 4.3 a general description is given of the elements implemented into the commercial FE-package DIANA. In section 4.4 several test problems, showing the different features of the elements, are compared with analytical solutions. In section 4.5 the compression of a schematic motion segment is discussed 


\subsection{Equations}

The following three coupled partial differential equations have to be solved:

$$
\begin{array}{ll}
\text { momentum } & : \vec{\nabla} \circ Q-\vec{\nabla} p=\overrightarrow{0} \\
\text { continuity } & : \vec{\nabla} \circ \vec{\nabla}^{s}-\vec{\nabla} \bullet[K \circ \vec{\nabla}(p-\omega)]=0 \\
\text { diffusion } & :(\dot{\mathrm{e}})_{s}+\vec{\nabla} \circ \circ\left(\vec{v}^{f}-\vec{v}^{5}\right)=\frac{1}{n^{f}} \vec{\nabla} \circ[D \circ(\vec{\nabla} \varrho+\xi \vec{\nabla} c p g)] \\
\text { osmotic retention : } \sigma=\phi R T\left(2 c+c^{p 8}\right)
\end{array}
$$

with

g the Cauchy stress tensor of the solid

(") the material time derivative following the solid

$\vec{v}$ the velocity of the fluid

$v^{3}$ the velocity of the solid

$\mathrm{n}^{\mathrm{f}}$ the volume fraction of the fluid

$c^{\text {pis }}$ the proteoglycan concentration

c the mobile anion concentration
$\mathrm{K}$ the permeability tensor

$\mathrm{R}$ universal gas constant

$T$ absolute temperature

$\phi$ the osmotic coefficient

D the diffusion tensor

$p$ the fluid pressure

e mobile ion density

The primary unknowns are the displacement of the solid, the fluid pressure and the ion density. The ion concentration and ion density are related by the molecular mass. Compared to chapter 3 some abbreviations are used: the subscript 'eff' for the effective stress and the superscripts " $i$ " and '-" for the ions and anions respectively are omitted. The relative fluid flow is represented by $\overrightarrow{v^{t}}-\overrightarrow{v^{3}}$.

The ionized proteoglycans are the origin of the fixed charge concentration. As the proteoglycans are entangled in the collagen network the concentration varies with the deformation. Both the solid and the fluid are intrinsically incompressible: a volume change is only achieved by expelling fluid out of the tissue. The fixed charge concentra* tion in the deformed state is expressed as:

$$
c^{p g g}=\frac{\text { total amount } P G}{V^{f}}=\frac{c_{0}^{p g} V_{0}^{f}}{V_{0}^{f}-(1-J) V_{0}^{m}}=\frac{c_{0}^{p g}}{1-\frac{(1-J)}{n_{0}^{f}}}
$$

with the subscript o denoting the reference state

I the volume change

Using the same derivation as for $\mathrm{c}^{\mathrm{p}}$, the porosity in the deformed state is given by:

$$
n^{f}=1-\frac{1-n_{0}^{f}}{J}
$$

The equations (4.1) until (4.3) form the set of equations describing the triphasic swelling behaviour of intervertebral disc tissue. The initial conditions and the boundary conditions. 
are given by the Donnan equilibrium results:

$$
\begin{aligned}
2 c_{b} & =-c^{p g}+\sqrt{\left(c^{p g}\right)^{2}+4 f^{2} c^{2}} \\
p_{b} & =\phi R T\left(2\left(c_{b}-c\right)+c^{p g}\right)
\end{aligned}
$$

with $\bar{c}$ the external salt concentration

$\mathrm{c}_{\mathrm{b}}$ the salt concentration at the boundary within the tissue

$p_{b}$ the fluid pressure at the boundary within the tissue

$\mathrm{f}^{2}$ the mean activity coefficient quotient

The boundary condition for the momentum of the mixture is:

$$
(\text { a }-p D \circ \vec{n}=\vec{t}
$$

with $\overrightarrow{\mathrm{t}}$ the boundary stress vector

$$
\bar{n} \text { the unit normal vector on the boundary }
$$

The boundary conditions depend on the local proteoglycan and ion concentrations. These concentrations change during deformation. Hence, the boundary conditions are deformation dependent.

\subsection{The weighted residual method}

By taking the dot vector product of the local equilibrium condition (4.1.1) with an arbitrary vector $\bar{w}$ and by multiplying the continuity $(4.1 .2)$ and diffusion (4.1.3) equation by the arbitrary scalar function $\mathrm{g}$ and $\mathrm{h}$ respectively, and integrating this result over the deformed mixture volume $\mathrm{V}$ the weighted residual form of (4.1) is obtained:

$$
\begin{aligned}
& \int_{V}(\vec{\nabla} \circ \underline{\underline{V}}-\vec{\nabla} p) \circ \vec{w} d V=0 \\
& \int_{V}\left[\vec{\nabla} \circ \vec{V}^{s}-\vec{\nabla} \circ[K \circ \vec{\nabla}(p-\vec{\sigma})]\right] g d V=0 \\
& \int_{V}\left[n f(\dot{\varrho})_{s}+n f \vec{\nabla} \varrho \circ(\vec{v} f-\vec{v})-\vec{\nabla} \circ\left[D \circ\left(\vec{\nabla} \varrho+\xi \vec{\nabla} c^{p g}\right)\right] \mid h d V=0\right.
\end{aligned}
$$

Because $\vec{w}, g$ and h are arbitrary functions, $(4.1)$ and $(4.6)$ are equivalent. First $(4.6 .1)$ is alaborated. With the identity:

$$
(\vec{\nabla} \circ \underline{Q}-\vec{\nabla} p) \circ \vec{w}=\vec{\nabla} \circ\left[(\alpha-p D \circ \cdots \vec{w}]-(\alpha-p D:(\vec{\nabla} w))^{c}\right.
$$

and by applying Gauss' theorem (4.2.1) is transformed to:

$$
\int_{V}\left(\alpha-p D:(\vec{\nabla} \vec{w}) c d V=\int_{A} \vec{n} \circ(\alpha-p D) \propto \vec{w} d A\right.
$$

with

$$
\begin{aligned}
& \text { A the boundary surface of the mixture } \\
& n \text { the outward unit normal on } A
\end{aligned}
$$


Generally, both the volume $V$ and the surface $A$ are a priori unknown, as they change in time. Calculation of the integrals is simplified by transforming them to the reference volume $\mathrm{V}_{0}$ with outer surface $\mathrm{A}_{0}$ (total Lagrange description):

$$
\int_{V_{0}}\left(a-p D \cdot\left(E^{-c} \cdot \overrightarrow{\nabla_{0}} \vec{w}\right)\right)^{c} J d V=\int_{A_{0}} \vec{n}_{0} \circ E^{-1} \circ(a-p D \circ w) d A
$$

with $J=\operatorname{det}(\underline{F})$ the volume change

$\bar{n}_{0}$ the reference outward unit normal on $A$

$\nabla_{0}$ the gradient operator with respect to the reference state

Using the identities:

$$
\begin{aligned}
A:(B \circ C) & =(A \circ B): C \\
A^{c}: B^{c} & =A \cdot B \\
(A \circ \vec{a}) \circ \vec{b} & =A: \vec{a} \vec{b}
\end{aligned}
$$

equation (4.9) becomes:

$$
\int_{V_{0}}\left[\left(E^{-1} \underline{e}-p E^{-1}\right):\left(\nabla_{0} \vec{w}\right)^{\natural}\right] J d V=\int_{A_{0}} \vec{t} \circ \tilde{w} d A
$$

with $\overrightarrow{\mathrm{t}}$ the boundary stress vector defined as: $\overrightarrow{\mathrm{t}}=\left(\mathbb{F}^{-\varepsilon_{0}} \overrightarrow{\mathrm{n}}_{0}\right) \circ\left(\sigma-\mathrm{p}\left[\mathrm{J}_{\mathrm{A}}\right.\right.$

$J_{A}$ the surface change defined as: $J_{A}=J\left\|E^{-\infty} \circ \vec{n}_{0}\right\|^{-1}$

At this point the symmetrical second Piola-Kirchhoff stress tensor $\$$ is introduced, related to the Cauchy stress tensor $\underline{q}$ according to:

$$
S=J \underline{F}^{-1}{ }^{\circ} \underline{Q} \circ E^{-c}
$$

Substituting (4.12) into (4.11) yields:

$$
\int_{V_{a}}\left(\vec{\nabla}_{0} \tilde{w}\right) c:\left[S \circ E^{c}-p J E^{-1}\right] d V=\int_{A_{0}} \vec{t} \circ \vec{w} d A
$$

Equation (4.13) is non-linear in the displacement and pressure. Because of this nonlinearity it cannot be solved directly: an iterative solution procedure is needed. The Newton-Raphson technique is chosen. Suppose that at a certain time estimates of the displacement field and the pressure field are available. In general these estimates, marked by an asterisk $(*)$, differ from the actual fields satisfying (4.13). The difference between the estimated and actual situation is marked by delta $(\delta)$ : 


$$
\begin{aligned}
& \vec{u}=\vec{u}+\delta \vec{u} \\
& p=\vec{p}+\delta p \\
& \vec{t}=\vec{t}+\delta \vec{t} \\
& S=\vec{S}+\delta \underline{S} \\
& E=\vec{E}+\delta E \\
& J=J+\delta J
\end{aligned}
$$

Assuming that the differences between the actual and estimated values are small compared to the actual values we derive:

$$
\begin{aligned}
& \stackrel{*}{E}=I+\left(\vec{\nabla}_{0} \vec{u}\right)^{c} \\
& E^{-1} \approx\left(I-E^{-1} \circ \delta E\right) \circ E^{-1} \\
& \delta E=\left(\vec{\nabla}_{0} \delta \vec{u}\right)^{c} \\
& J \quad \approx J\left(1+E^{-1}: 8 F\right) \\
& \delta S=\frac{\partial S}{\partial E}: \delta E \\
& \delta E=\frac{1}{2}\left(\delta E^{c} \circ E+E^{c} E^{c} \delta E\right)
\end{aligned}
$$

Substituting (4.14) and (4.15) in (4.13) yields after linearizing and rearranging terms:

$$
\begin{aligned}
& \int_{V_{0}}\left(\vec{\nabla}_{0} \vec{w}\right)^{c}: S \circ \delta E^{c} d V+\int_{V_{0}}\left(\vec{\nabla}_{0} \vec{w}\right)^{c}:\left(E^{c} \circ \frac{\partial S}{\partial E} \circ D: \delta E^{c} d V\right. \\
& -\int_{V_{0}}\left(\vec{\nabla}_{0} \vec{w}\right)^{c}:\left[E^{-1} J \delta p+\left(\left(E^{-1}: \delta E E^{-1}-E^{-1} \cdot \delta E \circ E^{-1} \mid p J\right] d V=\right.\right. \\
& -\int_{V_{0}}\left(\vec{\nabla}_{0} \vec{w}\right)^{c}:\left(S \circ E^{c}-E^{-1} p J\right) d V \\
& +\int_{A_{0}} \vec{w} \circ \vec{t} d A+\int_{A_{0}} \vec{w}_{0} \delta \vec{t} d A
\end{aligned}
$$

As only estimations are left the asterisk is omitted. Before discretizing the equilibrium equation (4.16), analogous mathematics is applied to the continuity equation (4.6.2). Using Gauss' theorem and rewriting with respect to the undeformed configuration yields:

$$
\int_{V_{0}}\left[g\left(E^{-c} \circ \vec{\nabla}_{0}\right) \circ \vec{\nu}^{s}+\vec{\nabla}_{0} g^{\circ}\left(E^{-1} \circ K^{\prime} \circ E^{-c}\right) \circ \vec{\nabla}_{0}(p-\omega)\right] / d V=\int_{A_{0}} g q^{f} d A
$$

with $\mathrm{q}^{\mathrm{i}}=\left(\mathrm{E}^{-c} \circ \overrightarrow{\mathrm{n}}_{0}\right)(\mathrm{K} \circ \vec{\nabla}(\mathrm{p}-\varpi)) \mathrm{J}_{\mathrm{A}}$ representing the fluid sounce at the boundary surface 
Because finite rotations should not influence the permeability, $\underline{\mathrm{K}}$ must be neutralised for rigid body rotations:

$$
\underline{K}=\underline{R} \cdot \underline{R}_{0} \circ \mathbb{R}^{\boldsymbol{c}}
$$

with $\quad \underline{R}$ the rotation tensor from the polar decomposition of the deformation gradient tensor $\underline{E}=\underline{R} \circ \underline{U}, \underline{U}$ representing the stretch tensor.

$\underline{K}_{0}$ the rotation neutralized permeability tensor

Introducing the difference between the estimated and actual fields in the same way as in (4.14) and linearizing yields for (4.17):

$$
\begin{aligned}
& \int_{V_{0}} g\left[\left(E^{-\epsilon} \circ \vec{\nabla}_{0}\right) \circ \delta \vec{v}^{s}+V: \delta E\right] J d V+\int_{V_{0}} \vec{\nabla}_{0} g \circ K_{u} \circ \vec{\nabla}_{0} \delta p J d V+ \\
& \int_{V_{0}} \vec{\nabla}_{0} g \circ N^{\circ} \cdot \vec{\nabla}_{0} \delta \varrho J d V=\int_{A_{0}} g q^{f} d A+\int_{A_{0}} g \delta q^{f} d A- \\
& \int_{V_{0}}\left[g\left(E^{-c} \circ \vec{\nabla}_{0}\right) \cdot \vec{v}^{s}+\vec{\nabla}_{0} g \circ \underline{K}_{u} \circ \vec{\nabla}_{0}(p-w)\right] J d V
\end{aligned}
$$

with $\underline{V}$ and $\underline{N}$ known tensor functions over $V_{0}$ depending on deformation, pressure and ion concentration

$\underline{\mathrm{K}}_{\mathrm{a}}=\underline{\mathrm{U}}^{-1} \circ \underline{\mathrm{K}}_{0} \circ \underline{\mathrm{U}}^{-\mathrm{e}}$ the Lagrangian permeability tensor

The diffusion equation $(4.6 .3)$ is treated in the same way, including the rigid rotation neutralization of the diffusion tensor $\mathbb{D}$, and results into:

$$
\begin{aligned}
& \int_{V_{0}} h Z: \delta E J d V+\int_{V_{0}} h \vec{\mu} \circ \vec{\nabla}_{0} \delta p J d V \\
+ & \int_{V_{0}}\left[h n^{f} \delta(\dot{Q})_{s}+\vec{\nabla}_{0} h \circ D_{u} \circ \vec{\nabla}_{0} \delta \varrho+h n^{f}\left(E^{-c_{0}} \circ \vec{\nabla}_{0} \delta \varrho\right) \circ\left(\vec{v}^{f}-\vec{v}^{s}\right)\right] J d V= \\
& \int_{A_{0}} h q^{\prime} d A+\int_{A_{0}} h \delta q^{\prime} d A \\
- & \int_{V_{0}}\left[h n^{f}\left\{(\dot{\mathrm{e}})_{s}+\left(E^{-c_{0}} \vec{\nabla}_{0} \mathrm{e}\right) \circ\left(\vec{v}^{f}-\vec{v}^{s}\right)\right\}+\vec{\nabla}_{0} h \circ D_{u} \circ\left(\vec{\nabla}_{0} \mathrm{e}+\xi \vec{\nabla}_{0} c^{p g}\right)\right] J d V
\end{aligned}
$$

with $\bar{\mu}$ known vector function over $\mathrm{V}_{0}$ depending on deformation, pressure and ion concentration

Z known tensor function over $\mathrm{V}_{0}$ depending on deformation, pressure and ion concentration

$\mathrm{q}^{i}=\left(\mathrm{E}^{-\mathrm{c}} \circ \overrightarrow{\mathrm{n}}_{0}\right) \circ(\underline{\mathrm{D}} \circ \vec{\nabla}(\mathrm{c})) \mathrm{I}_{\mathrm{A}}$ representing the ion flux across the boundary surface $\mathrm{D}_{u}=\underline{\mathrm{U}}^{-1} \circ \underline{\mathrm{D}}_{0} \circ \underline{\mathrm{U}}^{-\mathrm{e}}$ the Lagrangian diffusion tensor

The equations (4.16), (4.19) and (4.20) are the linearized weighted residual equations. 


\subsection{Discretization}

\subsubsection{Spatial discretizaton}

The mixture volume is divided in sub-volumes of a relatively simple shape: the elements. The volume and surface integrals are replaced by the sum of the volume and surface integrals over the elements. It is assumed that an element always consists of the same solid material points at all time. Therefore, its shape will change in time. The position vector $\vec{x}$ of any material point within the element is expressed as a linear combination of a limited number of discrete points, the nodal points:

$$
\vec{x}=\varphi^{\prime}\left(\xi_{\alpha}\right) \vec{x}^{j} \quad ; \quad j=1, \ldots n_{u}
$$

$\varphi^{\prime}$ the shape function

$\xi_{\alpha}$ the material coordinates; $\alpha=1,2,3$

$\vec{x}^{j}$ nodal coordinates

$n_{H} \quad$ number of geometric nodal points

Summation convention is used: a double index denotes summation with respect to all its values. A Latin index refers to a nodal number. The displacement field is interpolated in the same way as the geometry. The pressure and concentrations fields are also approximated within an element by means of shape functions:

$$
\begin{aligned}
& \vec{u}=\varphi^{\prime} \vec{u}^{j} \quad ; j=1, \ldots n_{u} \\
& p=\psi^{s} p^{s} ; s=1, \ldots n_{p} \\
& \mathrm{e}=\chi^{w} \mathrm{Q}^{w} ; w=1, \ldots n_{c}
\end{aligned}
$$

$\vec{u}^{j}$ the displacement of node $j$

$p^{s}$ the pressure of node $s$

$c^{\text {w }}$ the concentration of node $w$

$\varphi^{j} \quad$ displacement shape function

$\Psi^{*} \quad$ pressure shape function

$x^{\text {wo }}$ concentration shape function

The interpolation functions are dependent on the material coordinates and chosen in such a way that:

$$
\begin{aligned}
& \varphi^{\prime}\left(\xi^{i}\right)=8^{w} \\
& \psi^{s}\left(\xi^{r}\right)=8^{s} \\
& \chi^{w}\left(\xi^{y}\right)=8^{w w}
\end{aligned}
$$


The number of independent nodal points for the displacement, pressure and concentration fields are given by $n_{u}, n_{p}, n_{c}$, respectively. The nodes for the different fields may coincide.

\subsubsection{Time discretization}

Besides the spatial discretization a time discretization is needed. Several integration schemes are available. The scheme to be used, has to fulfil two requirements. First it has to be unconditionally stable: errors in the primary unknowns or the"r derivatives at time t, do not grow during the integration. This type of stability is assured if the time step is small enough. However, if the time step is chosen too small, spatial instabilities immediately after loading occur, which disappear when time increases. Generally, for each implicit integration scheme the derivative of a function $y(t)$ is expressed as:

$$
\dot{y}(t)=A y(t)+f(v(\tau), \dot{y}(\tau), \dot{y}(\tau) ; \tau<\tau)
$$

The spatial instabilities are suppressed either by a small value of the integration constant A or a small element size (Vermeer, 1981 ). By choosing the pressure interpolation function one order lower as the displacement interpolation function the spatial instabilities still occur, however the instabilities do not increase when decreasing the time step (Snijders, 1986). Ranking common integration schemes in order of increasing value of A. $\Delta t$, we find:

Central difference $\quad 1 / 2$

Houbolt third order $\quad 11 / 6$

Newmark 2

Willson 3

The central difference method has the smallest value of $\mathrm{A} \Delta \mathrm{t}$, but it is only conditionally stable. The Houbolt third order integration with a variable time step is used. A special starting procedure is needed for the first two time steps. The Houbolt method yields for an arbitrary function $y(t)$ :

$$
\dot{y}(t)=A y(t)+B y\left(t-\tau_{1}\right)+C y\left(t-\tau_{2}\right)+D y\left(t-\tau_{3}\right)
$$

with $r_{i}$ previous time distance

$A, B, C, D$ integration constants depending on $\tau_{i}$

By such a definition the iterative change in the time derivative is given by:

$$
\delta \dot{y}=A \delta y
$$

\subsubsection{Discretization of the weighted residual equations}

Following the strategy giwen by Galerkin the weighting functions $\bar{W}, \mathrm{~g}$ and $\mathrm{h}$ are interpolated in the same way as $\vec{u}, p$ and $c$, respectively:

$$
\begin{aligned}
& \vec{w}=\varphi^{i} w_{a}^{\prime} \vec{g}_{a} \\
& g=\psi^{r} g^{r} \\
& h=\chi^{*} h^{v}
\end{aligned}
$$

$\overline{\mathrm{g}}_{\alpha}$ are the covariant base vectors of a curvilinear coordinate system in the material coordinate system $\left(\xi_{1}, \xi_{2}, \xi_{3}\right)$, while $w_{c}^{\mathrm{i}}$ are the contravariant components. Hence, the 
nodal displacement $\vec{u}^{j}$ is expressed in the same way. Again the summation convention is used for the greek index, referring to a base direction. Further elaboration of the weighted residual equations is eased as the integrands become independent of nodal quantities $u_{\beta}^{j}, p^{s}, c^{k}, w_{m}^{i}, g^{r}$, and $h^{v}$. Substituting $(4.22),(4.26)$ and $(4.27)$ in $(4.16)$, (4.19) and (4.20) yields:

$$
\begin{aligned}
w_{\alpha}^{l}\left[\left(I_{\alpha \beta}^{i j}+G E_{\alpha \beta}^{i j}+P_{\alpha \beta}^{i j}\right) \delta u_{\beta}^{j}-R_{\alpha}^{i s} \delta p^{s}\right. & \left.=\left(F_{e x t}\right)^{i}-\left(F_{i n t}\right)\right] \\
g^{r}\left[A R_{\beta}^{j r} \delta u_{\beta}^{j}+K^{w s} \delta p^{s}\right. & \left.=Q_{\alpha x t}^{r}-Q_{i n t}^{r}\right] \\
h^{w}\left[\left(A M^{n+w}+V^{n w}+D^{w w}\right) \delta Q^{w w}\right. & \left.=C_{e x t}^{w}-C_{i n t}^{v}\right]
\end{aligned}
$$

with the introduced abbreviations as the matrix representation of:

momentum:

$$
\begin{aligned}
& I_{\alpha \beta}^{i j} \quad=\int_{V_{0}^{e}}\left(Q_{\alpha}^{i}\right)_{0}^{c} \cdot S \circ\left(Q_{\beta}^{j}\right)_{0} d V \\
& G E_{\alpha \beta}^{i j}=\int_{V_{0}^{e}}\left(\phi_{\alpha}^{i}\right)_{0}^{c} \cdot\left(E^{c} \circ \frac{\partial S}{\partial E} \circ E\right):\left(\varphi_{\beta}^{j}\right)_{0} d V \\
& P_{\alpha \beta}^{i j}=-\int_{V_{0}^{2}} p\left[\left(\varphi_{\alpha}^{i}\right)_{0}^{c} \circ E^{-1}: E^{-1} \circ\left(\varphi_{\beta}^{j}\right)_{0}-\left(\varphi_{\beta}^{j}\right)_{0}^{c} \circ E^{-1}: E^{-1} \circ\left(\varphi_{\alpha}^{i}\right)_{0}\right] J d V \\
& R_{\alpha}^{i s} \quad=\int_{V_{0}^{e}}\left(Q_{\alpha}^{i}\right)_{0}^{c} \cdot E^{-1} \psi^{s} J d V \\
& \left(F_{i n i}\right)_{\alpha}^{i}=\int_{V_{0}^{i}}\left(Q_{a}^{i}\right)_{0^{\circ}}^{c}\left(S \circ F^{c}-p J E^{-1}\right) d V \\
& \left(F_{e x t}\right)_{\alpha}^{i}=\int_{A_{0}^{*}} \varphi^{i} \vec{t}_{\mathrm{m}} d A \\
& \left(\varphi_{\alpha}^{i}\right)_{0}=\vec{\nabla}_{0}\left(\varphi^{i} \vec{g}_{\text {䑶 }}\right) \\
& \vec{\psi}_{0}^{r} \quad=\vec{\nabla}_{0} \Psi^{r} \\
& \vec{x}_{0}^{v} \quad=\vec{\nabla}_{0} x^{v}
\end{aligned}
$$

flud flow:

$$
\begin{aligned}
& K^{m s}=\int_{V_{0}^{e}} \Psi_{0}^{r} \circ K_{u} \circ \mathbb{\psi}_{0}^{s} I d V
\end{aligned}
$$

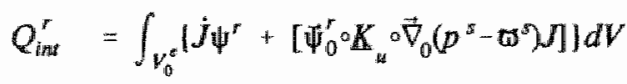

$$
\begin{aligned}
& Q_{e m p}^{r}=\int_{A_{0}^{*}} \psi^{r} q^{f} d A
\end{aligned}
$$


ion flux:

$$
\begin{aligned}
& M^{n+\infty}=\int_{V_{0}^{e}} n x x^{4} d d V
\end{aligned}
$$

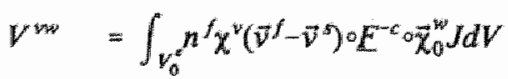

$$
\begin{aligned}
& D^{\text {w }}=\int_{V_{0}^{e}} \vec{\chi}_{0}^{*} \circ D_{\Delta} \circ \vec{\chi}_{0}^{*} J d V
\end{aligned}
$$

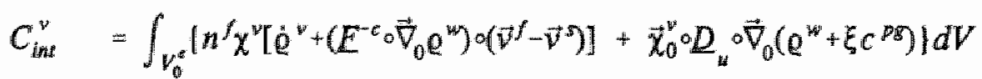

$$
\begin{aligned}
& C^{v}=\int_{A_{0}^{*}} x^{v} q^{v} d A
\end{aligned}
$$

In all three equations the iterative change of the external load is neglected. In the continuity equation $(4.19)$ the terms with $\delta \mathrm{Q}$ and $\delta \mathrm{F}$ and in the diffusion equation $(4.20)$ the terms with $\delta E$ and $\delta p$ are neglected. The weighting functions $w_{x x}^{i}, g^{r}$ and $h^{v}$ are still arbitrary: the equations between square brackets form a set of linear equations in the nodal unknowns:

$$
\left[\begin{array}{ccc}
S & R & 0 \\
R & -\frac{1}{A} & 0 \\
0 & 0 & D
\end{array}\right]\left[\begin{array}{c}
\delta \tilde{u} \\
\delta \tilde{p} \\
\delta \tilde{Q}
\end{array}\right]=\left[\begin{array}{c}
\tilde{n} \\
-\frac{1}{A} \tilde{q} \\
\tilde{z}
\end{array}\right]
$$

The continuity equations are divided by $-\mathrm{A}$ to obtain a symmetrical stiffness matrix if the diffusion equations are omitted. The column $\delta \tilde{u}$ contains all nodal iterative displacements, the column $\delta \tilde{p}$ all nodal iterative pressures and the column $\delta \tilde{e}$ all iterative concentrations.

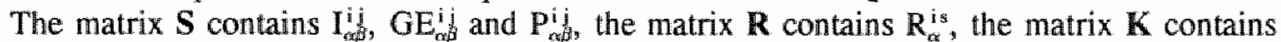
$K^{r s}$, while the matrix $\mathbf{D}$ contains $A M^{v w}$ and $V^{v w}$ and $D^{v w}$. The right hand side of (4.32) represents the difference between the calculated internal and external force $\tilde{\mathrm{n}}$, mass flow $\tilde{\mathrm{q}}$ and ion flux $\tilde{z}$ due to the estimated displacement, pressure and concentration field. Based on (4.32) it is possible to obtain an improvement of the estimated nodal quantities, as is explained next. First (4.32) is shortened to:

$$
M \delta \tilde{x}=\tilde{r}
$$

with $\quad$ M the total stiffness matrix

$\delta \tilde{x}$ the iterative correction on the nodal unknowns

imbalance or residual load

The time domain of interest is divided in a number of time steps. Suppose at a certain time $t=t_{\mathrm{n}}$ ( $n$ the increment number) all nodal quantities are known. The next state at $t=t_{n+1}$ is calculated as follows. The time increment $\Delta t_{n+1}$ is prescribed and the first estimation of the nodal quantities at $t=t_{n+1}$ is given by their values at $t=t_{n}$ :

$$
\begin{aligned}
& t_{m+1}=t_{n}+\Delta t_{n+1} \\
& \tilde{x}_{n+1}^{0}=\tilde{x}_{n}
\end{aligned}
$$


The superscript on the nodal quantities is the iteration number. A new estimate of the time derivatives (4.25) is calculated, a predictor right hand side $\vec{r}_{n+1}^{0}$ and the stiffness matrix $M_{n+1}^{0}$ are calculated (4.29-31). The tterative correction on the nodal unknowns and a new estimate are given by:

$$
\begin{aligned}
& 8 \tilde{x}^{1}=\left(M_{n+1}^{0}\right)^{-1} \cdot \ddot{P}_{n+1}^{0} \\
& \ddot{x}_{n+1}^{1}=\ddot{x}_{n+1}^{0}+\delta \tilde{x}^{1}
\end{aligned}
$$

With this new approximation a new residual load column, the corrector $\tilde{\mathbb{I}}_{\mathrm{m}+1}^{1}$ is calculated. In order to provide some indication of when the nodal unknowns and the internal forces and fluxes are near their equilibrium values an energy convergence criterion is used. From the second iteration onward the product of the nodal unknowns with the residual load column is compared to the value of the first iteration. Convergence is assumed to be reached when (Bathe, 1982):

$$
\left(\delta \tilde{x}^{2}\right)^{T} \cdot \tilde{r}_{n+1}^{i-1} \leq \in\left(\delta \tilde{x}^{\mathbb{1}}\right)^{T} \cdot \tilde{F}_{n+1}^{0}
$$

with $\quad \epsilon$ the tolerance for accuracy check

If this criterion is not satisfied a new iteration step like (4.32) is needed. If it is satisfied a new time step may be performed. This procedure is called the incremental iterative Newton-Raphson process.

\subsubsection{Boundary conditions}

The deformation dependent boundary conditions are accounted for by means of a least square method. Introducing penalty factors the following sum is minimized with respect to the free parameters $\mathrm{p}$ and $\mathrm{g}$ in each nodal point:

$$
S=\lambda_{1}\left(p-p_{b}\right)^{2}+\lambda_{2}\left(\varrho-e_{b}\right)^{2}
$$

with $e_{b}$ the ion density at the boundary according to (4.4.1)

$\mathrm{p}_{\mathrm{b}}$ the pressure at the boundary according to $(4.4 .2)$

$\lambda_{1}$ and $\lambda_{2}$ the penalty factors

The resulting stiffness matrix and right hand side are given by:

$$
\left[\begin{array}{ll}
\lambda_{1} & 0 \\
0 & \lambda_{2}
\end{array}\right]\left[\begin{array}{l}
p \\
Q
\end{array}\right]=\left[\begin{array}{l}
\lambda_{1}\left(p-p_{b}\right) \\
\lambda_{2}\left(\mathrm{e}-\mathrm{e}_{b}\right)
\end{array}\right]
$$

\subsection{Element formulation}

For the numerical studies iso-parametric elements of the serendipity family are used. To maximize the applicability of the elements, each of the three features (sollid deformation, fluid flow and ion diffusion) is optional. For instance to anallyze diffusion in a solid deforming medium the fluid flow is switched off. An Eulerian diffusion is obtained by 
setting all nodal displacements equal to zero and eventually prescribing a convective velocity if the medium is a fluid.

Two dimensional plane strain, three dimensional and axisymmetric elements hawe been developed. The order of interpolation of the different fields is determined by the originating equations (4.1). Looking at the momentum equation (4.1.1) one should interpolate the stress and the pressure in the same way. In a first order approximation the stress is proportional to the first order derivative of the displacement (Hooke's law). Hence, the pressure should be interpolated one order lower than the displacement. However the continuity equation $(4.1 .2)$ shows that the velocity should be interpolated in the same way as the pressure gradient, indicating that the pressure should be interpolated one order higher than the displacement. These two demands are in conflict. We decided to interpolate the displacement and pressure in the same way. The diffusion equation (4.1.3) is also parabolic. Thus all three fields are interpolated in the same way. In addition, this has computational advantages. Integration of the volume and surface integrals is carried out by means of the Gauss-integration. For the implementation of the software the commercially available (DIANA Analysis B.V., Delft, the Netherlands) finite element package DIANA (Borst et al., 1985), developed by TNO-JBBC (Delft, the Netherlands), is used. In the next section several test problems showing the features of the elements are presented. The numerical results are compared with the analytical solution. If no dimensions are specified, the SI-units are assumed and only the numerical values used, are giwen.

\subsection{Selected problems}

\subsubsection{Dispersion in laminar flow: Taylor dispersion}

The specific problem concerns the fate of a sharp pulse of solute injected in a long thin tube of length $L$ and radius $R$, filled with solvent flowing in a laminar flow. As the solute pulse mowes through the tube it is dispersed by convection and diffusion. When assuming that the mass transport of the solutes is only determined by radial diffusion and axial. convection an analytical solution is available (Taylor, 1953, Aris, 1956):

$$
C(z, t)=\left\{\begin{array}{l}
\frac{M / \pi R^{2}}{\sqrt{4 \pi E}} e^{-\left(z-v_{0} r^{2} / 4 E t\right.} \\
\frac{1}{\pi R^{2}} \int_{0}^{R} 2 \pi c(r, z, t) r d r
\end{array} ; E=\frac{\left(R v_{0}\right)^{2}}{48 D}\right.
$$

in which $\mathrm{M}$ is the total solute mass in the pulse, $\mathrm{z}$ the distance along the tube, $\mathrm{r}$ the radial coordinate, $v_{0}$ the fluid velocity, $D$ the diffusion coefficient, $E$ the dispersion coefficient, $C$ the concentration averaged over the cross-section, $c$ the local concentration and $t$ the time. To suppress the axial diffusion a transversely isotropic diffusion model (radial $\mathrm{D}_{\mathrm{r}}=10$, axial $\mathrm{D}_{2}=0.01$ ) is used in the numerical simulation. The numerical model $\left(L=10, R=1, v_{0}=1\right.$, linear quadrilateral axisymmetric element, element size $0.1 \times 0.1$, constant time step $=0.1$ ) agrees well with the analytical solution (fig. 4.1). Improvement of the solution may be obtained by further decreasing the axial diffusion coefficient. 
However as a result spurious oscillations occur, which may by seen in a mild form in the solution for $\mathrm{z}=0$ (indicated by the arrow in fig 4.1). They may by suppressed by using a Petrov-Galerkin interpolation (upwinded weighting functions) (Gerritsma, 1990). The order of magnitude of the spurious oscillations is governed by the Peclet number. In the intervertebral disc the Peclet number is lower than the critical value, so the standard. Gallerkin interpolation is used.

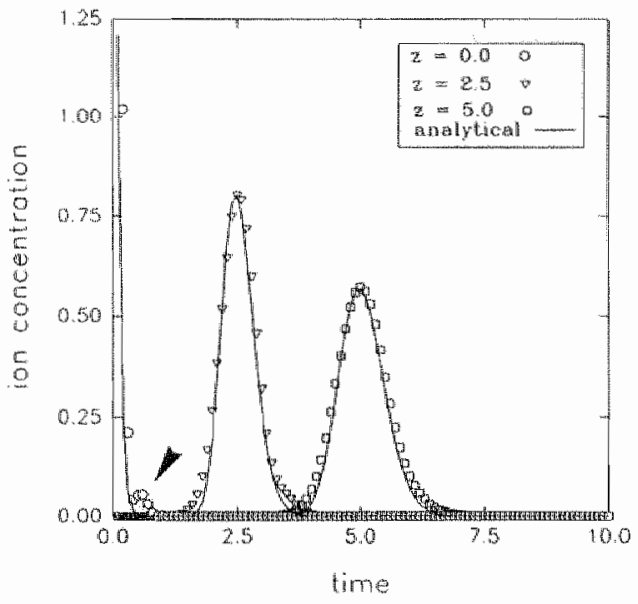

figure 4.1 Taylor dispersion. Both the analytical (lines) and numerical (markers) concentration profiles for $z=0, z=2.5$ and $z=5$ versus time.

\subsubsection{Finite inflation of a single-phase, thick, incompressible spheri- cal shell}

In order to obtain incompressible single-pliase material the permeability tensor $\mathbf{K}$ and the nodal mass flow $\tilde{\mathrm{q}}(4.28)$ are set to zero. To get a compressible single phase material all nodal pressures are set to zero. Consider a thick spherical incompressible shell (inner radius $r_{a, 0}$ and outer radius $r_{b, 0}$ ) subjected to a pressure $p_{i}$ along its inner surface and a zero pressure along its outer surface. The material behaviour of the solid matrix is given by a isotropic linear elastic relation between the second Piola-Kirchhoff stress tensor and the Green-Lagrange strain tensor (Young's modulus 2.0 MPa, Poisson's ratio 0.25). Upon inflation the sphere deforms into a sphere with inmer radius $r_{a}$ and outer radius $r_{b}$;

$$
\begin{aligned}
& r_{a}=\lambda_{a} r_{a, 0} \\
& r_{b}=\lambda_{b} r_{b, 0}
\end{aligned}
$$

The cavity pressure $\mathrm{P}_{\mathrm{cav}}$ for this spherical inflation is given by (Ogden, 1984):

$$
P_{\text {cav }}=\int_{\lambda_{a}}^{\lambda_{b}} \frac{d W] d \lambda}{\lambda^{3}-1} d \lambda
$$

with: W the strain-energy function 
Because of the symmetry about the plane $z=0$ only one half of the geometry is modelled. The mesh consists of 16 axisymmetric parabolic quadrilateral elements, 4 elements in the radial direction. The finite element solution for this problem is in good agreement with the analytical solution (fig. 4.2).

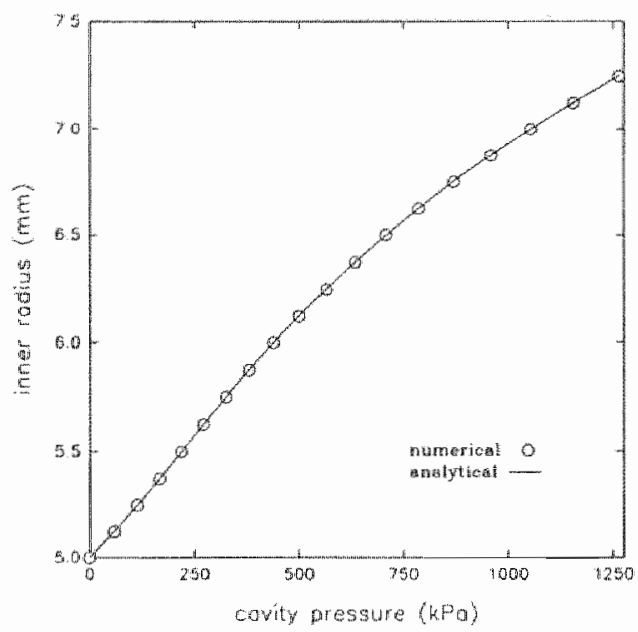

figure 4.2 Incompressible spherical shell. Both the anallytical (solid line) and numerical (markers) inflation versus cavity pressure.

\subsubsection{Linear unconfined compression}

In this paragraph the time dependent behaviour of a linear fluid-solid mixture is examined. Consider a cylindrical specimen of height $H$ and radius $R$, between two smooth, frictionless, impervious plates. The outer radial surface of the disc is stress free and the fluid pressure is zero. Hence, there is no resistance to the fluid flow at that boundary. The solid material is assumed linearly elastic with a constant permeability. The loading conditions are a constant strain rate for a time period $0<t<t_{0}$ followed by a stress relaxation for $t>t_{0}$. Using the small deformation theory and setting the Poisson's ratio to zero (which is no essential assumption) the resulting load history is given by (Armstrong, 1984):

$$
\frac{F(t)}{E_{s} \pi R^{2}}= \begin{cases}-\dot{\epsilon}_{0} t-\dot{\epsilon}_{0} \frac{R^{2}}{H_{A} K}\left[\frac{1}{8}-\sum_{n=1}^{\infty} \frac{1}{\alpha_{n}^{2}\left(\alpha_{n}^{2}-1\right)} e^{-\alpha_{n}^{2} \frac{H_{A} K t}{R^{2}}}\right] & t \leq t_{0} \\ -\dot{\epsilon}_{0} t_{0}+\dot{\epsilon}_{0} \frac{R^{2}}{H_{A} K} \sum_{n=11}^{\infty} \frac{1}{\alpha_{n}^{2}\left(\alpha_{n}^{2}-1\right)}\left[e^{\left.-\alpha_{n}^{2} \frac{H_{A} K t}{R^{2}}-e^{-\alpha_{n}^{2} \frac{H_{A} K\left(t-t_{0}\right)}{R^{2}}}\right]} t_{0} \leq t \leq \infty\right.\end{cases}
$$

$\alpha_{\mathrm{n}}$ are the roots of the characteristic equation $\mathrm{J}_{1}(\mathrm{x})-\mathrm{xJ} \mathrm{J}_{0}(\mathrm{x})=0$ where $\mathrm{J}_{0}$ and $\mathrm{J}_{1}$ are Bessel functions, $\mathrm{E}_{\mathrm{s}}, \mathrm{H}_{\mathrm{A}}$ and $v_{s}$ are the Young"s modulus, the aggregate modulus and the 
Poisson's ratio of the elastic solid matrix respectively, $K$ the permeability, $\mathfrak{E}_{0}$ the imposed strain rate.

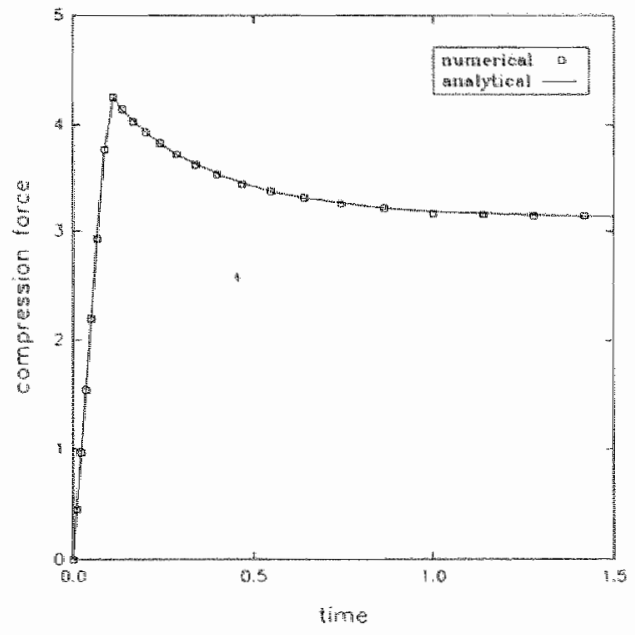

figure 4.3 Linear unconfined compression. Both the analytical (solid line) and numerical (markers) compression force versus time.

A numerical test $\left(\mathrm{H}=1, \mathrm{R}=1, \mathrm{E}_{\mathrm{s}}=1000.0, \nu=0.0, \mathrm{~K}=0.001, \mathrm{t}_{0}=0.1, \mathrm{\epsilon}_{0}=0.01\right)$ has been done using 14 axisymmetric linear quadrilateral elements. Because of the expected pressure gradient towards the outer radius a grading in the size of the elements from 0.12 to 0.027 is used. The time interval is divided into 25 time steps, the first one being 0.01 while the last one equals 0.14 . The numerical solution is in good agreement with the analytical solution (fig. 4.3).

\subsubsection{Non-linear confined compression}

The confined compression test is common in two-phase material research. In a confined compression test a sample of a fluid saturated porous material fits tightly in a impervious chamber. A mechanical load is applied through a porous filter connected to a metal shaft. The permeability of the filter is high compared to the permeability of the sample. In the case of soft tissue research a chemical loading is achieved by flowing a salt-water mixture through the filter. As we use this experimental set-up to determine the material behaviour of intervertebral disc tissue it is worthwhile to examine it in detail.

Consider the mechanical loading of a two-phase material (no sweiling phenomena). Assume no friction between the chamber and both the piston and the sample. Both the solid and fluid components are incompressible. The sample will deform only by expelling fluid through the filter. During the test a Heaviside load step is applied to the sample. Immediately after loading the load is balanced by the fluid pressure only. As the pressure equals zero at the filter a steep pressure gradient exits at the filter-sample interface. This pressure gradient causes the fluid to flow (Darcy's law), allowing the solid matrix to deform. By this process the balancing of the load is transferred from the fluid to the 
solid. Ultimately, the applied force is balanced by the solid only. This time dependent behaviour of a porous material is called consolidation.

Based on this type of experiment Holmes (1986) was able to determine the deformation dependent permeablity and Young's modulus of articular cartilage:

$$
\begin{aligned}
& K=k_{0}\left(\frac{J-n_{0}^{*}}{1-n_{0}^{*}}\right)^{2} e^{M E_{11}} \\
& s_{11}=H_{A}\left(\frac{\rho_{0}}{2 E_{11}+\rho_{0}}\right)^{1+\beta p_{0}} e^{2 \beta E_{11}} E_{11} \\
& \rho_{0}=1-\left(n_{0}^{5}\right)^{2}
\end{aligned}
$$

with $\quad E_{11}$ the Green-Lagrange strain in the axial direction

$\mathbb{S}_{11}$ the second Piola-Kirchoff stress in the axial direction

$K$ the permeability

$k_{0}$ the reference permeability

$\mathbf{n}_{0}^{\mathrm{s}}$ the volume fraction of the solid

$M, \beta$ material parameters

In the case of small deformations and the non-linear material behaviour described above an asymptotic analytical solution for the settlement of the piston for small values of $t$ is known (Holmes 1986):

$$
\begin{aligned}
\frac{u(x=H, t)}{u_{H}^{\infty}} & =\frac{2}{\sqrt{\pi}} \llbracket 1-\frac{2 u_{H}^{\infty}}{\pi H}\left(2+\frac{M}{2}-\frac{1}{1+n_{0}^{s}}\right) \rrbracket \sqrt{\frac{t}{\tau}} \\
u(x=H, t=\infty) & =u_{H}^{\infty} \\
\tau & =\frac{H^{2}}{k_{0} H_{A}}
\end{aligned}
$$

A numerical test using the experimental set-up and the material values derived by Holmes $(1986), H=1.6[\mathrm{~mm}], K=5.9510^{3}\left[\mathrm{~mm}^{4} / \mathrm{Ns}\right], \mathrm{M}=4[-], \mathrm{H}_{\mathrm{A}}=1 / 3[\mathrm{MPa}], B=0.0558[-]$, $n_{0}^{6}=0.2[-], \quad \tau=1290[\mathrm{~s}]$, has been done using 14 axisymmetric linear quadrilateral elements. In order to keep the small strain assumption valid the imposed strain is $1 \%$. Because of the expected pressure gradient towards the filter a grading in the size of the clements from $0.12 \cdot \mathrm{H}$ to $0.027 \cdot \mathrm{H}$ is used. The time interval $(2.5 \cdot \tau)$ is divided into 78 time steps, the first one being 0.5 seconds while the last one equals 775 seconds. The analytical and numerical solution are identical for the initial moments after "load application. The numerical solution gives also the transition to the steady state (fig. 4.4). 


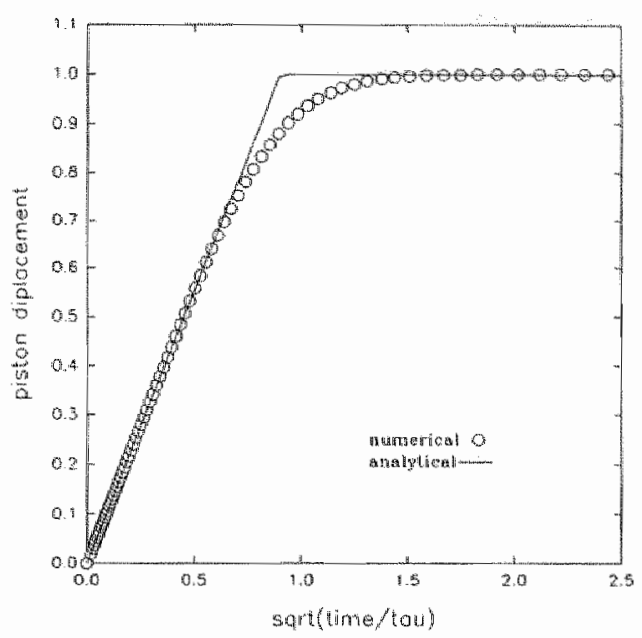

figure 4.4 Non-linear confined compression. Small strain theory combined with nonlinear material behaviour. Relative piston displacement versus square root of time.

Although no analytical solutions are available for large deformations, it is common in soft tissue research to strain the specimen up to decades of percents. In order to get some insight in the full non-linear behaviour of soft tissues we use the material behaviour determined by Holmes (1986). The specimen is strained to $25 \%$. From fig. 4.5 it is clear that the response of the tissue is governed by two mechanisms. An increase in stiffness results in a shift to a faster consolidation time. The increase in stiffness due to the nonlinear aggregate modulus (dotted line) is partly compensated by the decrease in geometrical stiffness (solid line). A decrease in permeability results in shift to a slower consolidation time. The solution of the geometrically and physically non-linear problem (dashed line) is close to the solution of the geometrically non-linear problem (dashed-dotted line). The effect of the non-linear stiffness is almost compensated by the effect of the non-linear permeability! The response of the tissue immediately after load application is linear in the square root of time, even if the permeability or the aggregate modulus are non-linear. The latter is not surprising as the physical non-linearities depend on the deformation. Deformation increases with time as fluid is expelled from the tissue. 


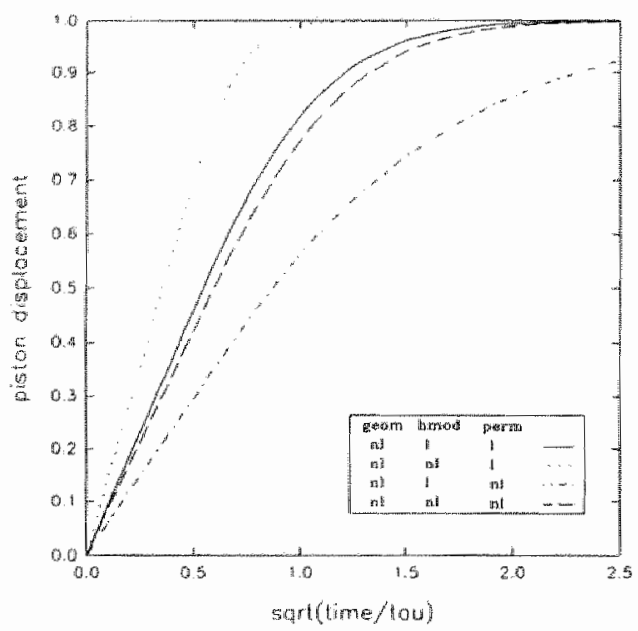

figure 4.5 Non-linear confined compression. Full non-linear response. Relative displacement of the piston versus the square root of time.

geom $=$ geometry; $\operatorname{hmod}=\mathrm{H}_{\mathrm{A}} ;$ perm $=\mathrm{k}_{0} ; \mathrm{nl}=$ non-linear; $\mathrm{l}=$ linear

\subsection{Compression of a schematic motion segment}

This application has no physiological meaning as the geometry and material properties are not chosen according to measured values and no anatomical details are modelled. The only function of this example is to show the possibilities of the numerical strategy. The intervertebral disc consists of a fibrous reinforced multi-layered mantle (anulus fibrosus) surrounding a randomly distributed network of collagen fibres (nucleus pulposus), both embedded in a proteoglycan matrix. The nucleus is modelled isotropic: Young's modullus 1.5 MPa, Poisson ratio 0.1. The orientation of the fibres in the anulus fibrosus is not modelled: the anulus is chosen isotropic with a Young"s modulus of $3.5 \mathrm{MPa}$ and Poisson ratio of 0.1. A uniform permeability of $9.010^{-4} \mathrm{~mm}^{7} \mathrm{MPa}^{-1} \mathrm{~s}^{-1}$ is used. In figure 4.6 the dimensions of the modelled intervertebral disc of the lower lumbar spine (LA-LS) are given.

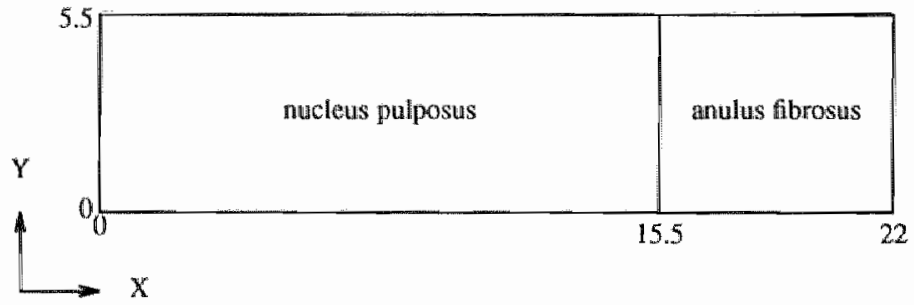

figure 4.6 Division of the tissue types and dimensions in $\mathrm{mm}$. 
The mesh contains 352 linear plane strain elements with 391 nodes. A grading towards the top and outer anulus is used. At he boundaries with fluid and ion exchange boundary elements are used (total 26). Both the base $(y=0 \mathrm{~mm})$ and the top $(y=5.5 \mathrm{~mm})$ and $x=0$ $\mathrm{mm}$ are planes of symmetry. At $\mathrm{x}=0$ the displacements in the $\mathrm{x}$-direction equal zero. At the base the displacements in the $y$-direction equal zero. Above the nucleus lymphatic activity yields that the symmetry condition on both the ion and fluid flow at the top is lost: flow is possible at this position. At the top the anulus is covered with a layer of cortical bone (not modelled). This layer is impermeable for both fluid and ions. Flutid flow is also possible across the outer anulus $(x=22 \mathrm{~mm})$. The proteoglycan concentration and porosity vary: constant in the nucleus and decreasing towards the boundary of the anulus. The distribution is chosen according to measured values (Urban and Maroudas, 1979). The resulting initial osmotic pressure profile is given in figure 4.7. This distribution is chemically in equilibrium with the external solution of physiological saline. Mechanical equilibrium while keeping the top supported in the $y$-direction is achieved by the steady state solution. Due to expansion in the x-direction of the disc, local redistribution (decrease) of the proteoglycan concentration occurs (fig. 4.7).

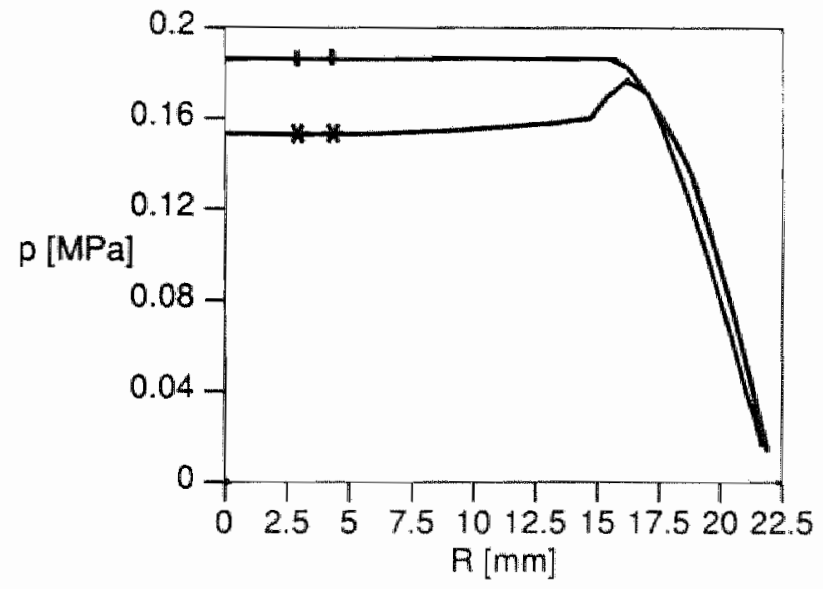

figure 4.7 Inital $(++)$ and equilibrium $(\mathrm{t}=0, * *)$ pressure distribution in $\mathrm{MPa}$ at the base $(y=0)$.

The stiffness difference between the anulus and nucleus results into a maximum in the proteoglycan concentration at the interface.

From this equilibrium $(t=0)$ the intervertebral disc is loaded by prescribing the displacement of the top in the $y$-direction according to:

$$
\begin{aligned}
u(t) & =u_{0} t & & 0<t<t_{0} \\
& =u_{0} t_{0} & & t>t_{0}
\end{aligned}
$$

We used $u_{0}=0.55[\mathrm{~mm}]$ and $t_{0}=900$ [s]. The resulting linear strain in the $y$-direction is $10 \%$. The total time period is divided into 57 time steps using the Euler backwards scheme. The first 900 seconds are divided into 9 steps, the time increment increasing from $10[s]$ to $275[s]$. The time steps for the relaxation period $(t>900[s])$ varied from 
10 to $500[\mathrm{~s}]$. In figure 4.8 the pressure distribution at the base is given. Due the nonuniform proteoglycan distribution an overshoot at the nucleus anulus interface occurs. In the following relaxation period the pressure drops. The resulting equilibrium pressure equals the osmotic pressure. Because fluid is expelled from the tissue, increasing the proteoglycan concentration, this pressure is higher at the one at $t=0$ [s]. In figure 4.9 this mechanism is demonstrated for the nodes at $x=0 y=0$ (node number 1), $x=0 y=5.5$ (node number 177) and $x=22 \quad y=0$ (node number 199). At the start of the relaxation period a pressure gradient in the $y$ direction exists. During the relaxation period this gradient vanishes. In the $x$-direction a pressure gradient is maintained according to the proteoglycan profile.

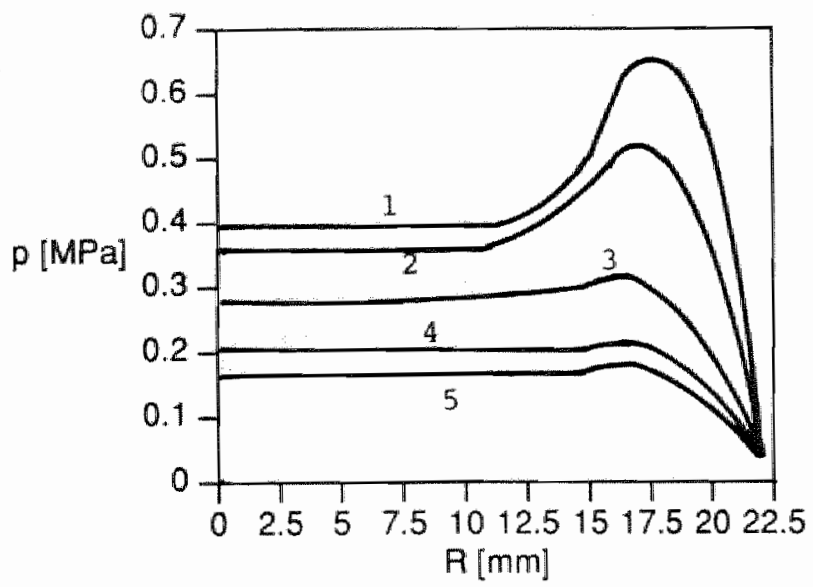

figure 4.8 Pressure [MPa] profile at the base for different times $t=0(5), 900(1)$, $1800(2), 5550(3), 18550(4)[s]$.

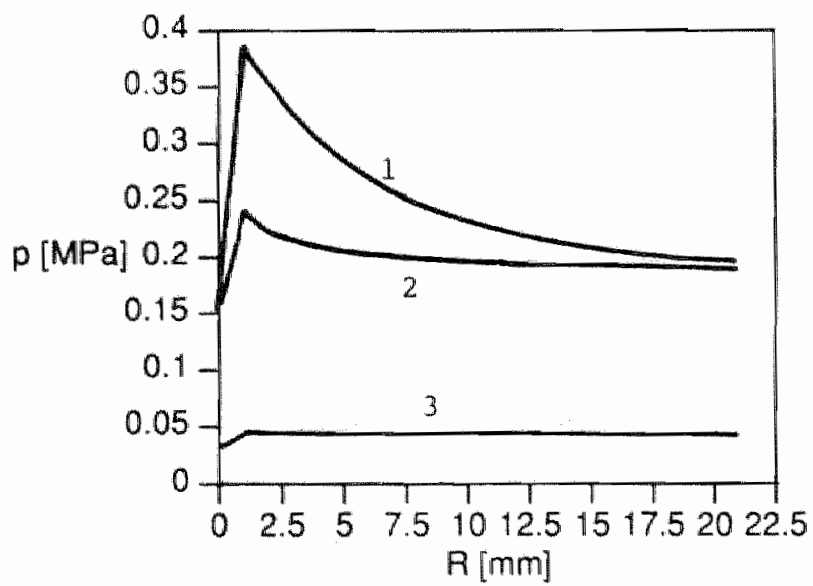

figure 4.9 Pressure [MPa] history for $x=0 y=0(1), x=0 y=5.5(2)$ and $x=22 y=0(3)$ 


\subsection{References}

Aris, R. Procedings of the Rayal Society of London, Series A, 235:67, 1956.

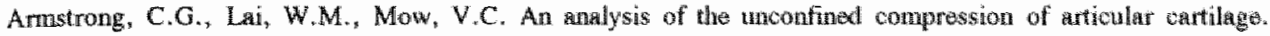
ASME J. Biomech. Eng., 106:165-173, 1984.

Bathe, K.J. Finite element procedures in enginering andysis. Prentice-Hall, Englewood Cliffs, New Jorsey. 1982.

Borst, R. de, Kusters, G.M.A. Nauta, P., de Witte, F.C. DIANA - a comprehensive, but flexible funite alement system, in: Finite element systems: a handbook, ed. C.A. Brebbia, Springer Verlag, Berlin, 1985.

Brekelmans, W.A.M. Non-linear mechanics"numerical aspects. Lecture notes (in Dutch). Eindhoven Uniwersity of Technology, Eindlhoven, The Netherlands, 1988.

Gerritsma M.I. Artificial diffision methods in convection dominated flows, Internal report BL-90-209, TNO Institute for Building Materials and Struetures, 1900 .

DLANA User"s Manual, Linear Static Analysis. TNO Building and Construction Research, Delli, "The Netherlands, 1991.

DIANA User's Manuall, Nonlinear Analysis. TNO Building and Construction Reserach, Delf, The Netherlands, 1994 .

DIANA Programming. TNO Building and Construction Research, Delft, The Netherlands, 1992.

Hoimes, M.H., Lai, W.M. Mow, V.C. Singular pertubation analysis of the nonlinear, flow-dependent compressive stress relaxation behniour of articular cartilage. ASME J. Bionech. Eng., 107:206.218, 1985.

Holmes, M.H. $\mathbb{F}$ inite deformation of soft tissue: analysis of a mixhme model in unimaxial compression. ASME J. Biomexh. Eng, 108:372-381, 1986.

Huyghe, J.M.R.J. Non-linear finte element models of the beating left ventricle and the intramyocardial coronary circutation. PhD thesis, Eindhowen Unversity of Tecnollog, Eindhoven, The Netherlandis, 1986.

Ogden, R.W. Non-linear elastic deformations. Ellas Horwond Chicester, 1984.

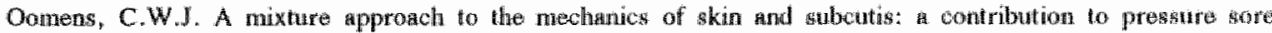
nesearch. PHD thessis. Twente University of Technology, Enschede, The Netherllands, 1985.

Snijders, J.M.A. The mechanical behaviour of sof biological tissine based on a mixture nowol (in Duteh). MSe thesis, Eindliovent University of Technology Eundhoven, The Natherlands, 1986.

Taylor, G.1. Proceedings of the Royal Society of Lomdon, Series A, 219:186, 1954.

Urban, I., Maroudas, A. The measurement of fixed charge density in the intervertebral disic. Biocheinica et Biophysica Acte, 586:166-18, 1979 .

Vermeer, P.A., Vermyt, A. An accurancy condition for consolidation by funite elentenks int. I. Numi. Ana. Meth. Geomech. 5:1-14, 1981.

Veldpans, F.E. Geometrically non-linear mechanics. Lecture nottes (ir Dutch). Eindhoven University of Technology, Eirdhoven, The Netherlands, 1988. 



\section{BIPHASIC VERSUS TRIPHASIC CONFINED COMPRESSION}

In chapter 3 a triphasic theory, based on thermodynamic principles, is presented. In order to test whether the proposed model is realistic one dimensional confined compression experiments on canine samples are performed. The samples are taken from the canine anulus fibrosus. The loading protocol consists of a combination of mechanical and chemical loading. After in introduction (section 5.0) the material and methods are depicted in section 5.1. Detailed information is given on the specimen preparation, the experimental set-up, the experimental protocol, the data analysis and the fitting procedure. As results (section 5.2) are given: the histology of the specimen, the displacement of the loading piston versus time, and the biphasic and triphasic material parameters. The chapter ends with a discussion about the obtained results. 


\subsection{Introduction}

In chapter 2 a detailed description of the anatomical and biochemical structure of the intervertebral disc and its different tissues is given. A brief outline will be presented here. The intervertebral disc consists of a fibrous reinforced multi-layered mantle (anulus fibrosus) surrounding a randomly distributed network of collagen fibres (nucleus pulposus), both embedded in a proteoglycan matrix. Because the proteoglycans are relatively immobile, osmotic effects play an important role, particularly if thetissue is in contact with a bathing solution. The disc osmotic pressure is highly dependent on the proteoglycan concentration (Urban et al., 1979, Urban and McMullin, 1985, 1988) and the salt concentration of the bathing solution (Myers et al., 1984). The post-mortem equilibrium pressure of nucleus pulposus specimen is about 0.1-0.3 MPa, both in human and dog (Urban and McMullin, 1985, 1988, Glover et al., 1991, Johnstone et al., 1992). The equilibrium pressure, measured in a complete motion segment, increases with the load: in pig nucleus $0.32 \mathrm{MPa}$ for a $50 \mathrm{~N}$ load and $0.56 \mathrm{MPa}$ for a $100 \mathrm{~N}$ load (Ohshima et all, 1989). Due to the osmotic swelling pressure, determination of the intrinsic material properties is cumbersome. Generally, two strategies are used to overcome these difficulties.

1. Using closed-loop control of the displacement, preventing the specimen to swell, until equilibrium is reached. In a second stage the actual test load is added to the swelling load. The displacement data during the second stage are analyzed using the biphasic theory (Mow et al, 1980), neglecting osmotic effects. Compressive stiffness and permeability of anulus fibrosus specimens have been determined in this way (Best et al., 1989, Guilak et al., 1990).

2. Allowing swelling to occur, but comparing the data with a triphasic theory, including osmotic effects. Triphasic theories have been developed for articular cartilage (Grodzinsky et al., 1981, Myers et al., 1984, Lai et al., 1991) and for intervertebral disc (Chapter 3, Snijders et al., 1992)

The goal of this study is to determine the time course of swelling and compression of specimens of canine anulus fibrosus under a combination of changing mechanical and chemical load for differences in spinal levels, regions and orientations in the disc. The design of the experiment is tailored for an one dimensional experimental yerification of triphasic theories.

\subsection{Material and methods}

\subsubsection{Specimen preparation}

Mongrel dogs of either sex having a body mass of $20-35 \mathrm{~kg}$ were sacrificed. The animals were used in cardiological experiments (Vos et al., 1990ab). It was assumed that the drugs used (ouabain, lidocain, doxorubicin or flunarizine) did not influence the chemical 
and mechanical characteristics of the intervertebral disc. The lumbar spine with the two adjacent thoracal vertebrae and the sacrum was dissected and within 1 to 2 hours after death frozen and stored for maximum one month at $-80^{\circ} \mathrm{C}$.

Samples were prepared batchwise as needed for testing while cooling with liquid nitrogen $\left(-196^{\circ} \mathrm{C}\right)$. Special care was taken to assure that the tissue stayed frozen at all time. Individual motion segments ( $1 / 2$ vertebra - disc - $1 / 2$ vertebra) were sawed by a bandsaw, the arcus removed. From the central region of the motion segment a strip of approximately $12 \mathrm{~mm}$. width was sawed in the dorso-ventral direction (figure 5.1 and figure 5.2). From the two lateral leftowers also a central strip with the same dimensions was sawed in the lateral direction. The three strips were reduced to square pieces of 8 $\mathrm{mm}$. Special care was taken to assure that the length axis of the strip was as perpendicular as possible to the collagen sheets. The height of the bone in the strip was reduced to approximately $2 \mathrm{~mm}$.

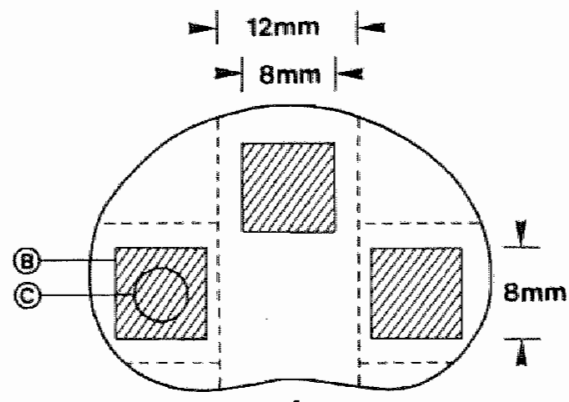

A
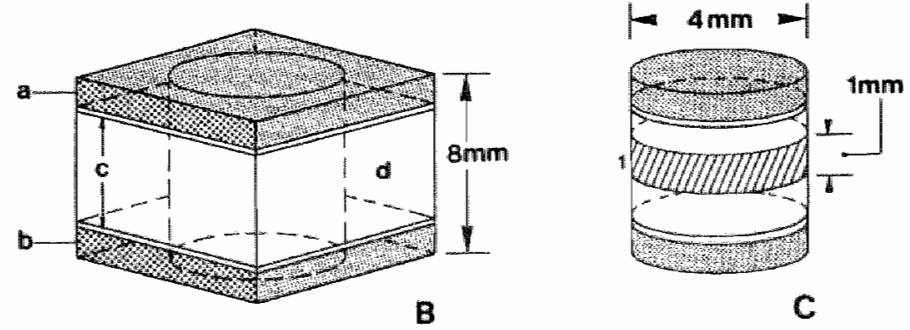

C

figure 5.1 Preparation of the specimen in the axial direction

A: top view of the intervertebral disc

B: $\quad 3-\mathrm{D}$ view of the square sample

C: 3-D view of the cylindrical sample as turned from the square sample; the specimen is cross hatched
$a-b$ : vertebrae
c: endplates
$d$ : anulus fibrosus

The actual test specimens were prepared as follows. The square samples were glued with Tissue-tek (OCT Compound 4583, Miles Diagnostics, IN, USA) in a cylindrical pipe holder (inner diameter $12 \mathrm{~mm}$ ) made of acrylate. The glued sample was cooled by 
dipping in liquid nitrogen to avoid shrink ruptures. Ultimate they were stored at least for 1 day at $-80^{\circ} \mathrm{C}$. The holder was clamped in a tuming lathe. During the whole preparation the sample was cooled with liquiid nitrogen. The turning procedure for an axial test specimen was as follows (figure 5.1). The vertebra and the bony and cartilage endplate were removed. The boundary vertebra-endplate was sharply marked due to vascularisation of the vertebra. In order to remove the endplate at least one millimetre of tissue was cut away. The front of the specimen was levelled. In the axial direction the specimen was tumed over a length of $4 \mathrm{~mm}$ to the desired diameter of $3.9 \mathrm{~mm}$. With a firmer chisel, with increased neutral edges, a specimen of $1 \mathrm{~mm}$ was cut off. During this procedure the specimen usually broke before the cutting was completed. The resulting small cylinder on the specimen was removed by a scalpel. The specimen was taken approximately from the midzone of the intervertebral disc.

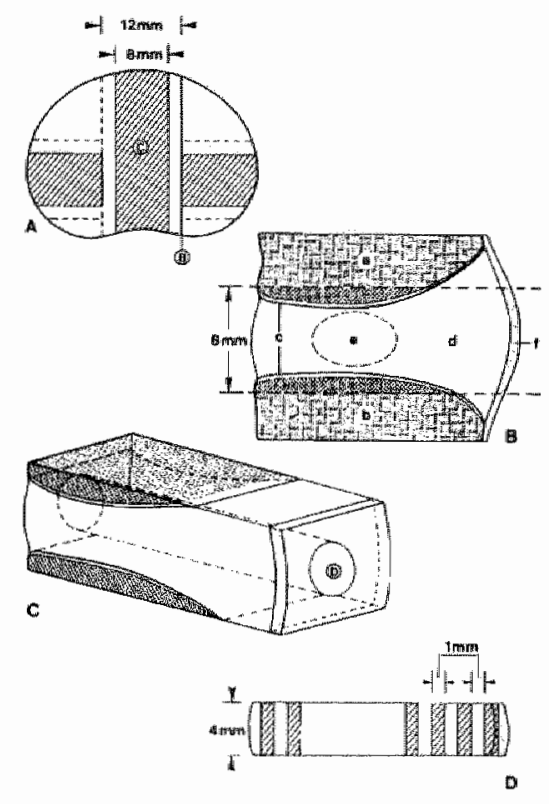

figure 5.2 Preparation of the radial specimens.

A Top view of the intervertebral disk, sawed strips are cross hatched.

B Side view of dorsoventral block

C 3-D view of dorsoventral block, turned cylinder (hatched)

D Side view of turned cylinder, test specimens cross hatched a: upper vertebra, b: lower vertebra, $c$ : endplates, d: anulus fibrosus, e: nucleus pulposus, f: anterior longitudinal ligament.

Radial test specimens were made in a similar way (figure 5.2). The dorsal part of the dorso-ventrall sample was deep in the holder, the anterior longitudinal ligament on its top. The sample was flattened by removing a minimal amount of tissue. The sample was turned to the desired diameter over a length of $4 \mathrm{~mm}$. The first test specimen (thickness 1 
mm) was cut off by a firmer chisel. This specimen contained mainly the anterior longitudinal ligament. The front of the sample was flattened again and the next sample was cut. Each specimen was cut only if the full diameter and height contained soft intervertebral disc tissue; no bony tissue was allowed.

Unfortunate this preparation method failed for the nucleus region. Each motion segment rendered in principle ventral, ventrolateral and lateral specimens. For the (ventro)lateral samples no distinction in left or right was made. No difference between the two was assumed. The spinal level, region and orientation were give in table 5.1. The actual test specimen were stored at $-80^{\circ} \mathrm{C}$ for maximum three weeks.

\begin{tabular}{|l|l|l|l|}
\hline L4-L5 & L5-L6 & L6-L7 & L7-S1 \\
\hline $1 A_{V L}$ & $2 A_{V}$ & $5 A_{V L} 1 A_{V}$ & $3 A_{L} 2 R_{V} 2 R_{L}$ \\
\hline
\end{tabular}

table 5.1 Spinal level, direction and region of the specimens.

direction: $A=A x i a l, R=$ Radial

region: suffixes $\mathrm{V}=$ ventral, $\mathrm{VL}=$ ventrolateral, $\mathrm{L}=$ lateral

The initial characterization of the tissue type was done visually. After the experiment a definitive characterization of the tissue was made histologically. After the experimentall session the test samples were fixed in $4 \%$ neutral formaldehyde, dehydrated in ethanol, embedded in G.M.A. (Technovit 7100, Kulzer, Wehrheim, Germany), cut at $3 \mu \mathrm{m}$, stained (thionine, $\mathrm{pH}=4.5$ ), and examined histologically.

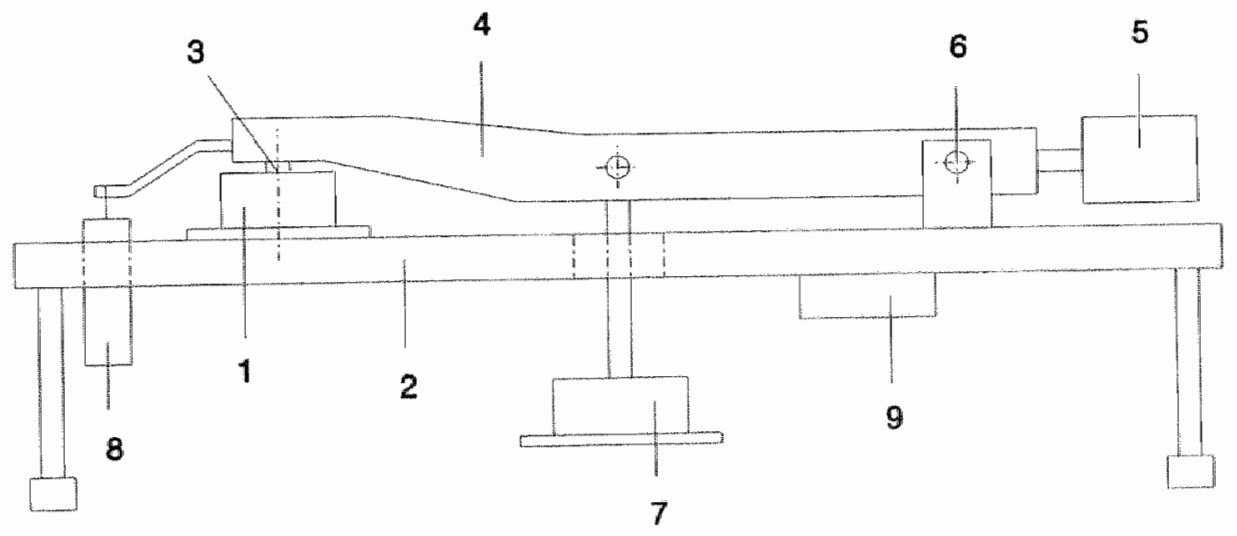

figure 5.3 Overview of the test apparatus

1: chamber; 2: table; 3 : loading piston; 4:loading arm; 5 : counter weight;

6: rotation axis; 7: weight; 8: LVDT; 9: vibrator. 


\subsubsection{Experimental set-up}

Uni-axial confined swelling and compression experiments were performed in a special testing chamber. In figure $5.3 \mathrm{a}$ schematic overview of the complete test apparatus is given. The test chamber (1) rested on a table (2), the interface was oiled. If, due to an excess of change in thickness of the specimen, the loading piston contacted the chamber wall, the chamber could move with as less as possible resistance relative to the loading piston.

The loading piston (3) was connected to a long loading arm (4), minimizing edge errors. In the unloaded situation the arm was balanced by a counter weight (5). The loading arm was connected to a rotation axis (6) with prestressed ball bearings, thus minimizing the friction moment. The actual mechanical loading was achieved by manually placing a calibrated weight on the scale (7). The scale was placed exact halfway the rotation axis of the loading arm and the loading piston, hence only half of the weight was counterbalanced by the test specimen. The loading arm was connected to a DC operated linear variable displacement transducer ( 8 , LVDT, Schaevitz) interfaced by a Labmaster $\mathrm{AD}$ converter to an IBM-AT. During the experiment the displacement of the piston was automatically recorded. The sample frequency was chosen between $0.033 \mathrm{~Hz}$ and 0.05 Hz. In order to avoid stick phenomena between the test specimen and the chamber wall the test apparatus was vibrated by a membrane pump (9) during 2 seconds, 0.5 second after each sample was taken. The membrane pump was also controlled by the Labmaster card.

In figure 5.4 a schematic drawing is given of the chamber. The mechanical load was applied to the tissue by means of a conic impervious stainless steel piston (a). The opening angle of the piston was chosen in such a way that for specimen with a thickness of about $1 \mathrm{~mm}$ no stick occurred during the swelling or compression stage. The maximum diameter of the piston was $3.94 \mathrm{~mm}$, while the diameter of the cylindrical impervious stainless steel chamber (b) was $4.00 \mathrm{~mm}$. To prevent any leakage of fluid between the piston and the chamber, the piston wall was greased with vaseline. The cylindrically shaped specimen (c), diameter $3.90 \mathrm{~mm}$ and height $0.87+0.33 \mathrm{~mm}$, rested on a sintered glass filter (d). The pore size lied between 16 and $40 \mu \mathrm{m}$; the permeability, measured in a classical Darcy set-up was $10^{12} \mathrm{~m}^{4} / \mathrm{Ns}$. Through the filter the specimen was in contact with a bathing solution. By altering the ionic strength of the solution the specimen was loaded chemically. The bathing solution was recirculated through the filter, thus minimizing the influence of stagnant films on the transport of ionic solutes into the tissue. The temperature of the solution was controlled to $19 \pm 2^{\circ} \mathrm{C}$. Two different bathing solutions were used. As both fluid circuits were equivalent only one is discussed in detail. The actual circulation was driven by the hydrodynamic pressure caused by the elevation of

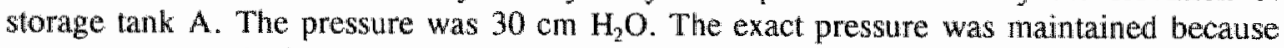
storage tank A hadl an overflow in the second storage tank B. The outflow from the testing chamber also flowed also in storage tank B. The bathing solution was pumped from storage tank B into storage tank A. By manipulating different valves the bathing solution was changed. Mixed solutions flowed into a waste tank. Homogeneous solutions were circulated. A vacuum pump, under pressure $85 \mathrm{kPa}$, freed the filter and the pipes from air, thus optimizing the contact between the bathing solution and the sample. 


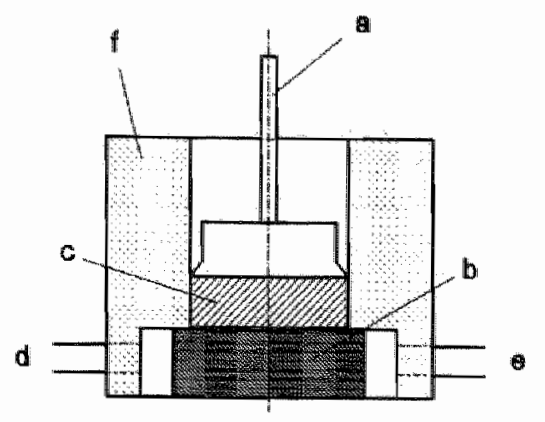

figure 5.4 Schematic view of the testing chamber

a: loading piston; $b$ : porous filter; $c$ : specimen; d: fluid inlet; e: fluid outlet; f: wall

\subsubsection{Experimental protocol}

The test procedure given in this section was used for both the axial and radial test specimen. At least two hours before the actual testing the bathing solution was circulated through the test apparatus. A virtual homogeneous temperature was achieved. The loading piston was moved around the workpoint in order to check for any friction or stick. By resting the loading piston on the filter the zero thickness was recorded. The test specimen was stored in liquid nitrogen until it was placed in the testing chamber. The levelled side was placed on the filter and the shaft was lowered. While the specimen thaws it was loaded with a conditioning preload of $1.0 \mathrm{~N}(0.08 \mathrm{Mpa})$. The vacuum pump freed the filter from air. After 10 seconds the conditioning bathing solution, $0.6 \mathrm{M} \mathrm{NaCl}$, was circulated through the filter. In a period of about 1.5 minutes after the contact of the piston and the experimental slice a slow decrease in thickness occurred. During this time period the specimen thawed while the bathing solution entered the filter and the load was applied, resulting in the setting and swelling of the specimen. The minimum thickness was taken as initial height of the specimen. This period was followed by a rapid increase of thickness of the tissue.

\begin{tabular}{|l|l|l|l|l|}
\hline stage & a & b & c & d \\
\hline & conditioning & swelling & consolidation & control \\
\hline [NaCl] & $0.6 \mathrm{M}$ & $0.2 \mathrm{M}$ & $0.2 \mathrm{M}$ & $0.2 \mathrm{M}$ \\
\hline mechanical load & $0.08 \mathrm{MPa}$ & $0.08 \mathrm{MPa}$ & $0.2 \mathrm{MPa}$ & $0.08 \mathrm{MPa}$ \\
\hline
\end{tabular}

table 5.2 Experimental protocol. 
The experimental protocol consisted of four stages (table 5.2): conditioning (a), swelling (b), consolidation (c), and control (d). As the boundary conditions of the tissue in the animal was unknown, the specimen was furst equilibrated against a known chemical and mechanical load of $0.6 \mathrm{M}$ and $0.08 \mathrm{MPa}$ respectively. The conditioning stage lasted 3-5 hours. The second stage (swelling stage) was initiated by a step change in the molarity of the bathing solution to $0.2 \mathrm{M}$, while maintaining the same mecharicall load. During five minutes in which the bathing solution was transiting from a $0.6 \mathrm{M}$ to a $0.2 \mathrm{M}$ solution, recirculation was interrupted and the outflow was pumped into a waste tank. After this period a homogenous solution of $0.2 \mathrm{M}$ was assumed and recirculation resumed. Again a rapid increase in thickness occurred due to the swelling of the test specimen. Within 3.5 hours the specimen height reached a new plateau and a third stage was initiated by a step increase of the mechanical load to $0.2 \mathrm{MPa}$, while maintaining the chemical load at $0.2 \mathrm{M} \mathrm{NaCl}$. This consolidation stage reached equilibrium within 3-8 hours. The fourth stage consisted in a stepwise mechanical unloading to $0.08 \mathrm{Mpa}$, while the chemical load was kept at the same value. Within 4-11 hours equilibrium was reached. The chemical and mechanical load for this stage were identical to those of the swelling stage. By comparing the equilibrium thickness of both stages an indication of the autolyse and the lost of proteoglycans was obtained.

A typical result is given in figure 5.5. The total experimental time was 14-20 hours. The specimen was removed from the testing chamber by a special piston and stored in the formalin solution. The testing chamber, loading piston and the filter were cleaned after each test.
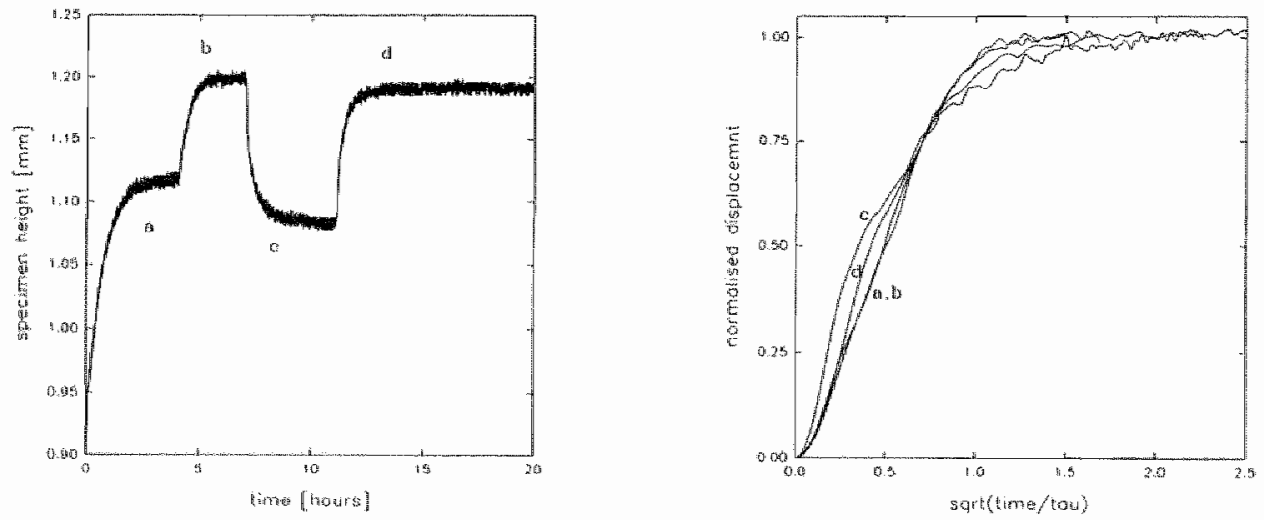

figure 5.5 Experimental data of a typical experiment

left raw data: height of the specimen versus time right normalised data stage a: conditioning stage $\mathrm{c}$ : consolidation stage $b$ : swelling stage d: control 


\subsubsection{Data analysis}

Before fitting the data to the non-linear triphasic equations of chapter 3 (Snijders et al., 1992) a rough evaluation of the relative time scale of the different phenomena was computed. The raw experimental data were smoothed by a generalized, cross-validatory spline (Woltring, 1986). Cubic natural B-splines were used. To be able to compare the smoothed experimental data of the different specimens they were normalized (figure 5.5) for each stage separately. The displacement was taken relative to the position at the beginning of the stage and divided by the relative equilibrium value. According to the results of chapter three, it is assumed that the response of the tissue is ruled by three coupled partial differential equations. However, at this stage it is uncertain which of the three dominates the response. Although the physical phenomena of the experiment cannot be described by means of the linear biphasic theory, for the state of quantifying the time scalle the linear biphasic consolidation time constant $\tau$ was used to normalise time. The consolidation time constant was quantified by fitting the data of each stage to the analytical solution of the linear biphasic theory (Terzaghi, 1923), for the sole purpose of scaling the time axis. For the displacement of the piston this results into:

$$
\begin{aligned}
U & =\frac{u(t)-u(t=0)}{u(t=\infty)-u(t=0)} \\
T & =\frac{t}{\tau} \quad M=\frac{\pi}{2}(2 n+1) \\
U(T) & =\sum_{n=0}^{\infty}\left(\frac{2}{M^{2}}\right)\left(1-e^{-M^{2} T}\right)
\end{aligned}
$$

with $\quad \mathrm{u}$ displacement of the piston

U normalised displacement of the piston

t experimental time

$T$ normalised time

The consolidation time constant calculated as described above was still a function of the specimen thickness. In order to overcome this limitation a transport coefficient ( $T$ ) was introduced. The transport coefficient was related to $t$ and the specimen height (h) by:

$$
\Upsilon=\frac{h^{2}}{\tau}
$$

The equilibrium height of the conditioning stage was used to calcullate the transport coefficients. In the simplified case of linear biphasic theory the transport coefficient is characteristic for the tissue involved; it equals the product of the aggregate modulus and the permeability.

\subsubsection{Fitting procedure}

In order to characterise the triphasic material behaviour the smoothed experimental data was fitted to the model as described in chapter 3 (Snijders et al., 1992). A standard fitting procedure compares the data with an analytical solution or an approximation of the experimental set-up. In order to derive a solution specific assumptions, for instance a homogenous strain field or heawyside loading conditions, are needed. Hence, the 
experimental freedom is lost or unrealistic boundary conditions are used. Even with the simplest boundary conditions and linear material behaviour no analytical solution for the triphasic behaviour of intervertebral disc tissue in a confined compression test is available. In order to overcome these limitations a numerical-experimental approach was used. For the numerical approach the Finite Element Method was chosen (chapter 4, Snijders et al., in press). The fitting procedure was based on a recursive minimum variance estimator. A detailed discussion of the method was given by Hendriks (1991), here only an outline based on our situation is given.

All experimental data (piston displacement versus time) is stored in column $\tilde{y}$. The quantitative behaviour of the tissue, represented by a finite set of unknown parameters, is stored in column $\tilde{\mathrm{x}}$. If there are no observation errors $\bar{y}$ is calculated for known $\tilde{\mathrm{x}}$. The observation errors are stored in column $\tilde{\mathrm{v}}$ and are assumed uncorrelated. The relationship between $\tilde{y}$ and $\tilde{x}$, characterized by $\tilde{\tilde{r}}$, is highly non-linear due to the physical and geometrical non-linear behaviour of the tissue. Summarizing, the model equation is written as:

$$
\tilde{y}=\tilde{r}(\tilde{x})+\tilde{v}
$$

with

$$
\begin{aligned}
& \tilde{y} \text { experimental data } \\
& \tilde{\mathrm{r}} \text { model relation } \\
& \tilde{\mathrm{x}} \text { material parameters } \\
& \tilde{v} \text { observation errors }
\end{aligned}
$$

The problem is to find the materiall parameters with an indication for their reliability for the observed data and their statistics. Suppose that an estimate $\tilde{x}_{\mathrm{k}}$ for $\tilde{x}$ is available. A new estimate can be found by minimizing the following quadratic form with respect to $\bar{x}$ :

$$
S=\left(\tilde{x}_{k}-\tilde{x}\right)^{T} \cdot\left(\boldsymbol{P}_{k}+\boldsymbol{Q}_{k}\right)^{-1} \cdot\left(\tilde{x}_{k}-\tilde{x}\right)+\left(\tilde{y}-\boldsymbol{R}_{k} \cdot \tilde{x}\right)^{T} \cdot \boldsymbol{M}^{-1} \cdot\left(\tilde{y}-\boldsymbol{R}_{k} \cdot \tilde{x}\right)
$$

with $\quad \tilde{\mathrm{x}}_{\mathrm{k}}$ an estimate for the material parameters

$\mathbf{P}_{\mathrm{k}}$ the variance matrix of the estimated parameters

$\mathbf{Q}_{\mathbf{k}}$ an arbitrary matrix

$\mathbf{M}$ the variance matrix of the observation errors

$\mathbf{R}$ the model matrix

The arbitrary matrix $\mathbf{Q}_{k}$ is unspecified, it may be interpreted as the variance matrix of the model errors. Usually it is determined by trail and error; it increases the convergence speed of the fitting procedure. The model matrix gives the sensitivity of the model on the parameters and is calculated numerically according to:

$$
\boldsymbol{R}_{k}=\frac{\partial \tilde{r}}{\partial \tilde{x}_{k}}
$$

Based on the reliability of both the estimated parameters and the observed data an new estimate $\tilde{x}_{k+1}$ is given by the following scheme: 


$$
\begin{aligned}
& \check{x}_{k+1}=\tilde{x}_{k}+\boldsymbol{K}_{k+1} \cdot\left\{\tilde{y}-\tilde{r}\left(\tilde{x}_{k}\right)\right\} \\
& \left.\boldsymbol{K}_{k+1}=\left(\boldsymbol{P}_{k}+Q_{k}\right) \cdot \boldsymbol{R}_{k}^{T} \cdot\left(M+\boldsymbol{R}_{k} \cdot\left(\boldsymbol{P}_{k}+Q_{k}\right) \cdot \boldsymbol{R}_{k}^{T}\right)\right\}^{-1} \\
& \boldsymbol{P}_{\boldsymbol{k}+1}=\left(\boldsymbol{I}-\boldsymbol{K}_{k+1} \cdot \boldsymbol{R}_{k}\right) \cdot\left(\boldsymbol{P}_{k}+\boldsymbol{Q}_{k}\right) \cdot\left(\boldsymbol{I}-\boldsymbol{K}_{k+1} \cdot \boldsymbol{R}_{k}\right)^{T}+\boldsymbol{K}_{k+1} \cdot \boldsymbol{M} \cdot \boldsymbol{K}_{k+1}^{T}
\end{aligned}
$$

with $\quad \mathbf{K}_{\mathrm{k}+1}$ the gain matrix

Convergence is assumed to be reached if the norm of the difference between the experimental and calculated data divided by the number of data points is small:

$$
\frac{\left(\tilde{y}-\tilde{r}\left(\tilde{x}_{k}\right)\right)^{T} \cdot\left(\tilde{y}-\tilde{r}\left(\tilde{x}_{k}\right)\right)}{n_{m}} \leq \epsilon
$$

with $\epsilon$ the tolerance for accuracy check

$\mathrm{n}_{\mathrm{m}}$ number of experimental data points

The fitting procedure was started using the first $\mathrm{m}$ data points. If convergence was achieved the number of data points was increased. A new fit based on the new data points was calculated. The number of data points was increased until at last a fit, based on the full data set, was obtained.

\subsection{Results}

\subsubsection{Histology}

Histological examination of the specimens shows no abnormalities, indicating that the preparation, especially the temperature treatment during the preparation and storage, does not cause visible damage. Due to the collagen fibres anisotropic shrink occurs during dehydration. All specimens consist of anulus fibrosus. In the axial specimens about 20 layers of collagen fibres are present, in the radial specimens 8-9. Two axial and one radial specimen are taken from the inner layers of the anulus fibrosus: over about $1 / 4$ of the area anulus fibrosus layers are not clearly visible.

\subsubsection{Displacement}

All specimens swell in the swelling and control stages (figure 5.6). Most specimens reach an equilibrium height in each stage. The equilibrium height during conditioning is reached in 2-6 h, during swelling in $2-4 \mathrm{~h}$ and in the control stage $2-4 \mathrm{~h}$. These durations however strongly depend on the sample thickness. During the consolidation and control stages the initial response was much faster than during swelling (table 5.3). 


\begin{tabular}{|l|l|l|l|l|l|l|}
\hline \multirow{2}{*}{ stage } & \multicolumn{5}{|l|}{$\begin{array}{l}\text { linear strain relative to } \\
\text { the conditioning stage [-] }\end{array}$} \\
\hline & axial & radial & all & axial & radial & all \\
\hline conditioning & $2.46 \pm 0.99$ & $5.05 \pm 1.21$ & $3.11 \pm 1.53$ & $0.16 \pm 0.04$ & $0.05 \pm 0.15$ & $0.13 \pm 0.09$ \\
\hline swelling & $\mathbb{2} .64 \pm 0.63$ & $2.84 \pm 1.11$ & $1.94 \pm 0.91$ & $0.08 \pm 0.03$ & $0.11 \pm 0.07$ & $0.09 \pm 0.04$ \\
\hline consolidation & $\mathbb{1 . 1 3 \pm 0 . 5 2}$ & $2.11 \pm 0.72$ & $1.38 \pm 0.70$ & $0.13 \pm 0.04$ & $0.21 \pm 0.08$ & $0.15 \pm 0.06$ \\
\hline control & $\mathbb{2} 1.21 \pm 0.60$ & 3.34 & $1.37 \pm 0.82$ & $0.11 \pm 0.03$ & 0.10 & $0.11 \pm 0.03$ \\
\hline
\end{tabular}

table 5.3 Experimental results for the four stages, mean and standard deviation axial $n=12$ for the four stages

radial $n=4$ for the first three stages, $n=1$ for the control stage

The linear strain of the radial specimen is greater than the strain of the axial ones during swelling and consolidation. This does not hold for conditioning, because one radial specimen decreased in height during this stage. This particular sample was taken from the outer anulus surface and contained for a part the anterior longitudinal ligament. The loss in height between the end of the swelling stage and the end of the control stage was $1.5 \pm 1.1 \%$ of the equilibrium height of the swelling stage. For only one of the radial specimen the control stage is measured.
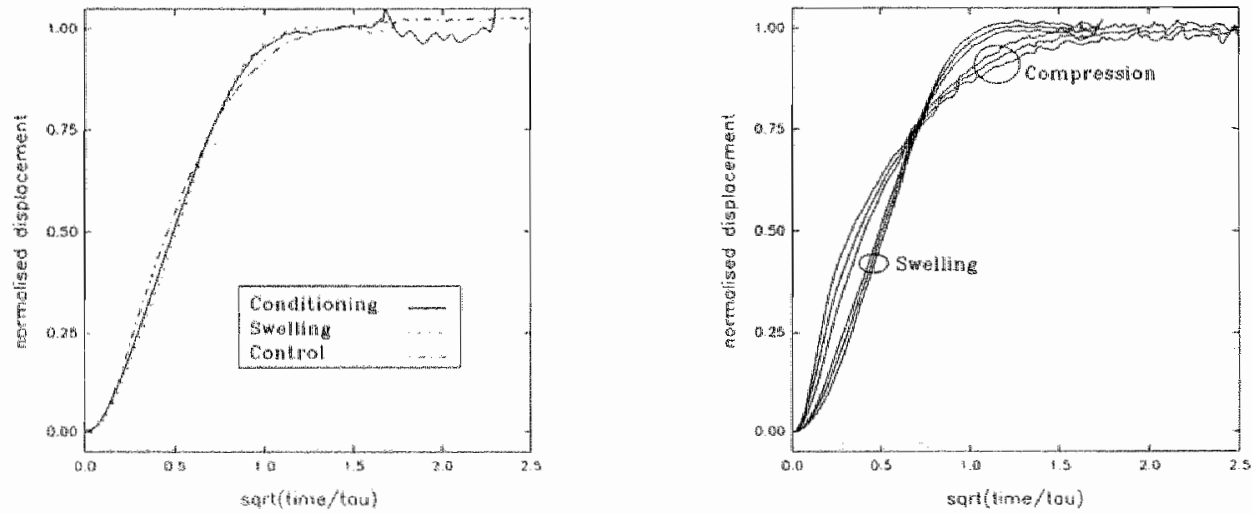

figure 5.6 Normalised data for the axial specimen. Left mean curves for conditioning, swelling and control stage. Right mean and standard deviation for the swelling and compression stage

From figure 5.6 it is clear that normalising the data as described above yields distinct and constant results for each stage taken separately. The normalised data show a great similarity for the three stages in which the thickness of the specimen increases (conditioning, swelling and control). In figure 5.6 the normalised data for the axial specimens are 
given for those stages. For normalised times $(t / \tau)$ between 0.01 and 0.8 a linear relation ship exists for the normalised displacement for those stages. For both the consolidation and the control stages the linear biphasic theory predicts a linear relationship (Holmes, 1985, 1986). Numerical results show that it even holds for non-linear biphasic behaviour (chapter 4, fig 4.5; Snijders et al., 1992). For the consolidation stage a rather parabolic curve is measured. Initially $(t / \tau<0.01)$ all curves are concave.

\subsubsection{Transport coefficient}

The transport coefficients of the conditioning and swelling stage differ from those of the consolidation and control stage (table 5.4 and 5.5). The transport coefficient of the conditioning stage and the swelling stage also differ, while those of the consolidation and control do not. These results hold for both the axial and all specimens. The radial specimens are not treated as a single group as their number is too low for a statistical analysis.

\begin{tabular}{|l|l|l|l|}
\hline stage & \multicolumn{2}{|l|}{ transport coefficient $\left[10^{-10} \mathrm{~m}^{2} \mathrm{~s}^{-1}\right]$} \\
\hline & axial & radial & all \\
\hline conditioning & $1.39 \pm 0.58$ & $0.50 \pm 0.33$ & $1.17 \pm 0.67$ \\
\hline swelling & $2.06 \pm 0.86$ & $0.97 \pm 0.75$ & $1.81 \pm 0.94$ \\
\hline consolidation & $3.14 \pm 1.56$ & $1.11 \pm 0.33$ & $2.64 \pm 1.61$ \\
\hline control & $3.17 \pm 1.94$ & 1.14 & $3.00 \pm 1.94$ \\
\hline
\end{tabular}

table 5.4 Transport coefficients for the four stages, mean and standard deviation

\begin{tabular}{|l|l|l|l|l|l|l|}
\hline \multirow{2}{*}{} & \multicolumn{2}{l|}{ swelling } & \multicolumn{2}{l|}{ consolidation } & \multicolumn{2}{l|}{ control } \\
\cline { 2 - 7 } & axial & all & axial & all & axial & all \\
\hline conditioning & $-2.82^{-*}$ & $-3.26^{-*}$ & $-3.06^{-\infty}$ & $-3.52^{\text {- }}$ & $-3.06^{-\infty}$ & $-3.18^{-\infty}$ \\
\hline swelling & & & $-2.98^{-\infty}$ & $-2.90^{-\infty}$ & $-2.5 \|^{*}$ & $-2.13^{*}$ \\
\hline consolidation & & & & & -0.16 & -0.31 \\
\hline
\end{tabular}

Table $5.5 \mathrm{Z}$-values of differences between transport coefficients of the four stages (Wilcoxon Matched-pairs Signed-ranks Test). Radial specimens are not tested for the low number of observations.

significance level:

$$
*=5 \% \quad *=1 \% \quad *=0.25 \%
$$

The transport coefficients for the axial and radial specimens differ significantly for the conditioning and swelling stage (table 5.5). As the tramsport coefficients of the consolida" tion and the control stage do not differ, they are pooled. These transport coefficients differ for the axial and radial specimens also. In the linear biphasic theory the 1 of the consolidation stage equals the product of the aggregate modulus $\left(\mathrm{H}_{\mathrm{k}}\right)$ and the permeability 
(K). The aggregate modulus is the quotient of the mechanical load and the resulting strain. For the consolidation sitage the averaged biphasic $H_{\text {A }}$ equals $1.01 \pm 0.31 \mathrm{MPa}$ for the axial and $0.66 \pm 0.30 \mathrm{MP}$ for the radial specimens. The permeability equals $3.2 \pm 1.5$ $10^{16} \mathrm{~m}^{4} /(\mathrm{Ns})$ and $1.8 \pm 0.510^{-16} \mathrm{~m}^{4} /(\mathrm{Ns})$ respectively. The aggregate modulus differ significantly ( $\mathrm{p}=0.04$, Mann-Whitney U-test) between the radial and axial specimens. The permeability does not differ significantly $(p=0.07)$. The biphasic theory is incapable of analysing the swelling stage. Based on the biphasic aggregate modulus and the swelling strain it is possible to estimate the equivalent osmotic load during the swelling stage. A change in the $\mathrm{NaCl}$ concentration from $0.6 \mathrm{M}$ to $0.2 \mathrm{M}$ results in a estimated osmotic load of $0.08 \pm 0.03 \mathrm{MPa}$ for the axial specimen and $0.06 \pm 0.02 \mathrm{MPa}$ for the radial specimens.

\begin{tabular}{|l|l|l|}
\hline conditroning & swelling & $\begin{array}{l}\text { consolidation }+ \\
\text { control }\end{array}$ \\
\hline$-2.55^{*}$ & $-2.18^{*}$ & $-3.00^{-*}$ \\
\hline
\end{tabular}

Table $5.6 Z$-values of differences of transport coefficients in axial and radial directions (Mann-Whitney $U$ Test). Values for consolidation and control were pooled, because they were not significantly different (table 5.5).

\subsubsection{Triphasic material properties}

The triphasic theory of chapter 3 (Snijders et al., 1992) is used for the analysis of the data of the swelling and control stages. The response of the tissue to either a mechanical or chemical load is the result of three different processes:

(1) Convection-diffusion of mobile ions, governed by the fluid flow relative to the solid $\left(\vec{v}-\vec{v}^{5}\right)$ and the diffusion coefficient tensor $(D)$. The ion concentration per unit tissue fluid (e) is given by:

$$
(\dot{\mathrm{Q}})_{s}+\vec{\nabla} \mathrm{Q} \circ\left(\overrightarrow{v^{f}}-\vec{v}^{3}\right)=\frac{1}{n^{f}} \vec{\nabla} \circ\left\{D \circ \vec{\nabla}\left(\mathrm{Q}+\xi_{c} c^{p 8}\right)\right\}
$$

During deformation the fluid to solid ratio changes, influencing the diffusion coefficient. In the $1-D$ situation this is accounted for by:

$$
D=\frac{n^{\prime}}{n_{0}^{f}} J D_{0}
$$

with $\mathrm{n}_{0}^{\mathrm{f}}$ the porosity in the reference state

$\mathrm{n}^{\mathrm{F}}$ the porosity in the deformed state

$\mathrm{J}$ the volume change

$\mathrm{D}_{0}$ the diffusion coefficient in the axial direction of the specimen in the reference state

(2) Large deformation of the fibre network and ground substance governed by: 


$$
\vec{\nabla} \circ \underline{\underline{a}}_{e f f}-\vec{\nabla} p=\overrightarrow{0}
$$

with $\alpha_{\text {eff }}$ the effective Cauchy stress tensor of the solid matrix

$\mathrm{p}$ the hydrodynamic fluid pressure

In the initial state of the tissue the osmotic pressure of the ground substance is counterbalanced by the tensile stresses in the solid. Therefore, the initial state is not stress-free. The Green-Lagrange strain $\mathrm{E}$ is defined relative to the a priori unknown unloaded state of the solid, representing the equilibrium state while in contact with a $0.6 \mathrm{M} \mathrm{NaCl}$ solution. The initial strain $E^{\prime}$ accounts for the difference of this state and the beginning of the swelling stage. Hence, this strain is generally different from zero and is one of the parameters to be fitted by the fitting algorithm. An exponential function is chosen for the deformation dependent stress-strain relation (Fung, 1973). For the 1-D situation the second Piola-Kirchhoff stress $S_{e f f}$ is coupled to the Green-Lagrange strain $E$ according to:

$$
S_{\text {eff }}=H_{A} e^{\beta E^{2}} E
$$

with $\quad \mathrm{H}_{\mathrm{A}}$ the initial aggregate modulus in the axial direction of the specimen $\beta$ material parameter

(3) Fluid flow relative to the solid phase:

$$
\vec{\nabla} \circ \vec{v}^{s}+\vec{\nabla} \circ\left(n^{f}\left(\vec{v}^{f}-\vec{v}^{s}\right)\right)=0
$$

For the classical biphasic Darcy's law the driving force for fluid flow is the pressure gradient. Here the fluid flow is driven by a pressure gradient and a concentration gradient of the mobile ions and the proteoglycans. Darcy's law is modified by a Donnan osmotic contribution to:

with $\quad \underline{K}$ the permeability tensor

$$
\begin{aligned}
n^{f}\left(\vec{v}^{f}-\vec{v}^{f}\right) & =-K \circ \vec{\nabla}(p-\tilde{\omega}) \\
\tilde{\omega} & =\phi R T\left(2 c+c^{p 8}\right)
\end{aligned}
$$

w the osmotic brake on the fluid flow

$\phi$ the osmotic retention

$\mathrm{c}^{\mathrm{pg}}$ the proteoglycan concentration

For the ideal Donnan situation the osmotic coefficient equals $2.4 \cdot 10^{4} \mathrm{Nm} / \mathrm{eq}$. An exponential function is chosen for the deformation dependent permeability (Oomens, 1985) which yields for the 1-D situation:

$$
K=K_{0} e^{M(J-1)}
$$

with $\quad \mathrm{K}_{0}$ the permeability in the axial direction of specimen in the reference state $M$ material parameter

The experiment, shown in figure 5.5, is fitted (figure 5.7). The calculated data lie within the noise of the experimental data. The fitted material parameters are: 


$$
\begin{array}{lll}
\mathrm{E}^{*}=-0.054[-] & \mathrm{H}_{\mathrm{A}}=0.47[[\mathrm{MPa}] & \beta=155.0[\mathrm{-}] \\
\mathrm{K}_{0}=9.5 \cdot 10^{-16}\left[\mathrm{~m}^{4} /(\mathrm{Ns})\right] & \mathrm{M}=18.5[-] & \mathrm{D}_{0}=1.72 \cdot 10^{-8}\left[\mathrm{~m}^{2} / \mathrm{s}\right] \\
\phi=0.41[-] & \mathrm{n}_{0}^{f}=0.81[-] & c_{0}=0.28 \cdot 10^{3}\left[\mathrm{eq} / \mathrm{m}^{3}\right] \\
\xi=0.0[-] & &
\end{array}
$$

From the material values obtained it is clear that the canine anulus fibrosus behaves

\begin{tabular}{|c|c|c|c|c|c|c|}
\hline \multicolumn{2}{|c|}{ swelling } & \multicolumn{5}{|c|}{ consolidation } \\
\hline & $\mathbb{H}_{A}$ & $\mathrm{~K}$ & $\Delta \pi$ & $\mathbb{H}_{\mathrm{A}}$ & $\mathrm{K}$ & $\Delta \pi$ \\
\hline & $\mathrm{MPa}$ & $10^{-16} \mathrm{~m}^{4} /(\mathrm{Ns})$ & $\mathrm{MPa}$ & $\mathrm{MPa}$ & $10^{-16} \mathrm{~m}^{4} /(\mathrm{Ns})$ & $\mathrm{MPa}$ \\
\hline biphasic & - & $=$ & 0.084 & 1.15 & 2.5 & - \\
\hline triphasic & 0.485 & 6.9 & 0.045 & 1.27 & 5.7 & 0.02 \\
\hline
\end{tabular}
physically non-linear, even for linear strains less than $10 \%$. In order to compare the linear biphasic and non-linear triphasic material properties, the latter are averaged over the stage. The results are given in table 5.7.

table 5.7 Biphasic and averaged triphasic aggregate modulus and permeability.

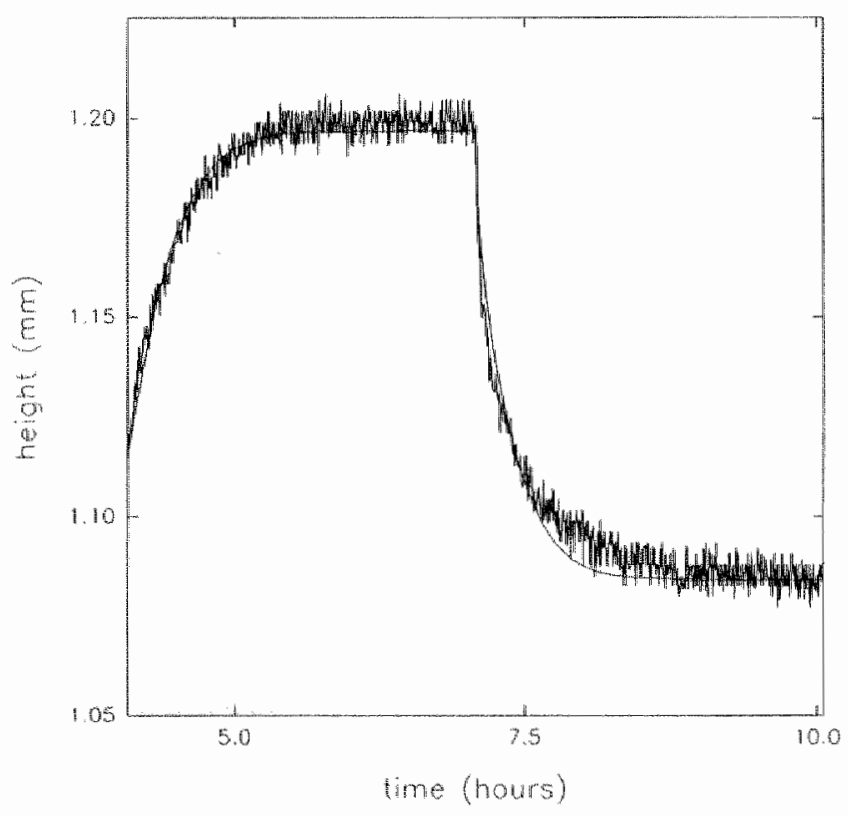

figure 5.7 Measured and calculated displacement by the triphasic model for the swelling and consolidation stages. 
The linear biphasic aggregate modulus and permeability during the swelling stage cannot be calculated. For the consolidation stage no change in osmotic pressure can be calculated in the biphasic theory. Again the linear biphasic aggregate modulus during consolidation is used to estimate the osmotic pressure change during the swelling stage. Generally the linear biphasic permeability is lower as the averaged triphasic one. The linear biphasic aggregate modulus is slightly smaller as the averaged triphasic one. Comparing the product of the averaged values of the triphasic aggregate modulus and permeability, and the biphasic transport coefficient, it is that the timescale of the proses is dominated by the fluid flow. By comparing the value of the osmotic coefficient it is clear that the osmotic effect of the specimen can not be modelled as ideal Donnan, $1.02 \cdot 10^{3} \mathrm{Nm} / \mathrm{eq}$ versus 2.4 $\cdot 10^{3} \mathrm{Nm} / \mathrm{eq}$. The estimated biphasic swelling pressure is almost wice the triphasic one. During consolidation $15 \%$ of the load is transferred by the osmotic pressure. The remaining $85 \%$ is counterbalanced by deformation of the solid matrix. The initial strain $\mathrm{E}^{\prime}$ is compressive. The diffusion coefficient is almost 500 times the value found by Maroudas (1975). The osmotic coefficient is half the value reported by Urban et al. (1979).

\subsection{Discussion}

This study demonstrates that the transient responses of anulus fibrosus tissue to a stepchange in mechanical or chemical loading produce distinct and consistent curves of normalised settlement versus square root of normalised time, irrespective of location and orientation in the disc. Except for small times $(T<0.01)$, the time course of the response agrees with the response as calculated with linear biphasic theory (Holnes, 1985, 1986). The response is linear for a much longer (normalised) time for all stages in which the height increases, then for consolidation. This might be due to a slowing down of the fluid flow, caused by a decreasing permeability and a increasing osmotic retention, with increasing time.

To our knowledge this is the first transient quantitative study on the triphasic behaviour of intervertebral disc tissue. The transient behaviour of the tissue is measured in a combination of chemical and mechanical loading conditions, as well in the axial as in the radial direction. A first assessment of the ability of a finite deformation finite elament model to fit the data is performed. The triphasic results show that at least the canine anulus fibrosus behaviour is physically non-linear, even for linear strains less than $10 \%$. The averaged triphasic and linear biphasic material properties differ. Considering that only the triphasic theory is able to describe the swelling behaviour, use of the biphasic theory for quantification of permeability and stiffness from consolidation data should be avoided, particularly when large strains are involved. Changes in proteoglycan concentration during the consolidation process due to fluid extrusion cause changes in osmotic pressure, that may not be negligible. Both the linear biphasic permeability and aggregate modulus are smaller as the iriphasic values. The estimated biphasic osmotic pressure change during swelling is almost twice the triphasic value. The definition of the biphasic swelling pressure is the total effective contribution, regardless the origin. The triphasic osmotic pressure change is totally accounted for by the presence of the small diffusible 
ions and the relative stagnant ionized proteoglycans. This is a Donnan approach. Lai et al. (1991) add to this an electrical repulsive force between the charged proteoglycan molecules, the so-called chemical expansion stress. Our preliminary results show no need for such contribution for intervertebrall disc tissue. Measurements including multi-axial deformation may influence this result in the future. Urban and Maroudas (1979) and Urban and McMullin $(1985,1988)$ come to a similar conclusion. However their results suggest that the mechanical load is counterbalanced by the fluid osmotic pressure. In our consolidation stage the osmotic pressure contributes for only $15 \%$ to the mechanical load, the remaining $85 \%$ is counterbalanced by tissue deformation. This result is comparable to the results of Lai et al. (1991). However their osmotic pressure includes a chemical expansion stress. Our osmotic coefficient is half the value found by Urban and McMullin (1985, 1988).

The transport coefficient for the strain levels measured, is an averaged value over the stage. The transport coefficient of the chemically loaded stages differ significantly from the mechanically loaded ones. The transport coefficient also differ significantly for the chemically loaded ones, while they do not differ for the mechanically loaded. In the mechanically loaded stages the external salt concentration is unchanged. The chemical load changes during the deformation as it results form a combination of the $\mathrm{NaCl}$ concentration and the proteoglycan concentration. However the transport coefficient is an averaged value: the effect for the consolidation and control stage is equal. For the first two stages the mechanical load is constant. As the strain level increases between these stages non-linear material properties influence the transport coefficient.

The linear biphasic transport coefficient and aggregate modulus differ significantly for the axial and radial specimens. For the permeability such conclusion can not be drawn on the data. As the linear biphasic transport coefficient equals the product of the aggregate modulus and the permeability, it suggests that the stiffness and permeability are not independent but related to each other. The permeability decreases as the volume fraction of the solid increases, resulting in an increase of stiffness. The difference of the aggregate modulus between the radial and axial specimen may be caused by the direction of the collagen fibres. As the fibres are approximately perpendicular to the radial direction, they do not significantly contribute to the radial stiffness, while they do contribute to the axial stiffness. The biphasic aggregate modulus is higher than previously published values: 0.38 MPa for non-degenerated and $0.18 \mathrm{MPa}$ for degenerated human anulus fibrosus (Best et al., 1989, Guilak et al., 1990). This might be due to the differences between human and canine discs.

Histologically no abnormalities are found. This indicates that the preparation and storage of the specimens do not cause visible damage. However from these results it is uncertain whether the material properties are affected. A number of researchers report for storage in $-20^{\circ} \mathrm{C}$ no differences in the material properties compared to fresh material (rabbit anulus fibrosus samples by Hickey and Hukins, 1979, complete motion segments by Panjabi et al., 1985 and canine articular cartilage by Kwan et al., 1992). As the specimens are stored at $-80^{\circ} \mathrm{C}$ and processed at $-196^{\circ} \mathrm{C}$ it is assumed that the material properties do not change.

It is believed that in the in-vivo situation the ionic environment of the tissue equals the ionic strength of a $0.15 \mathrm{M} \mathrm{NaCl}$ solution. The ionic environment during the conditioning stage is thus not physiological. The large range in osmotic strength of the external bath 
gives the opportunity to estimate osmotic effects accurately. Even against a $0.6 \mathrm{M} \mathrm{NaCl}$ solution the tissue swells in the conditioning stage (figure 5.5). Thus, it should be expected that the intervertebral disc is pre-stressed due to surrounding ligaments and muscles, which compensate for the disc osmotic pressure. One of the radial specimens shrinks in the conditioning stage. This particular sample is taken from the outer amulus: surface and contains for a part the anterior longitudinal ligament.

Proteoglycans may leach out from disc slices, when the latter are exposed to a salt solution (Urban and Maroudas, 1980). At present, no proteoglycan concentration measurements of the lest specimens have been made yet. A rough validation of the loss of proteoglycans during the experiment can be done on the basis of the loss of equilibrium height between the swelling and control stage. The decrease in equilibrium height between the swelling stage and the control stage is $1.5 \pm 1.1 \%$. The influence of these material losses on the calculated material parameters depends on the time course of the loss. The height changes during the equilibrium part of the swelling and consolidation stages are $+0.01 \% / \mathrm{h}$ and $-0.13 \% / \mathrm{h}$ respectively; the mechanical loads during these stages are 0.08 and $0.2 \mathrm{MPa}$. These values might suggest that the loss of proteoglycans into the filter occurs mainly at higher mechanical loads. It may be advisable to quantify the loss of proteoglycans by means of a biochemical analysis.

\subsection{References}

Best, B.A., Guilak F., Weidenbaum M., Mow W.C. Compressive stiffness and permeability of intervertebrall disc tissues: variation with radial position, region and level. Proceedings Winter Anunal Meeting ASME, San Francisco, 73-74, 1989.

Fung, Y.C. Biorheology of soft tisstues. Biorheology, 10:139-155, 1973

Glover, M.G., Hargens A.R., Mahmood, M.M., Gott, S, Brown, M.D., Garfin, S.R. A new technique for the in vitro measurement of nudeus pulposus swelling pressure. Journal of Orthopadic Research. $9: 61-67$, 1991.

Grodzinsky, A.J., Roth, V., Meyers, E., Grossman, W. D., Mow, V.C. The significance of electrome chancal and osmotic forces in the nonequilibrim swelling behavour of articular cartilate in thension. Journal of Biomechanical Engineering, $103: 221-231,1981$

Guilak, F., Zhu., W.B., Weidenbatum, M. Best, B., Mow V.C. Compressive material properties of human anulus fibrosus. in: Abstracts First World Congres Biomechanics 11, San Diego, 41, 1990.

Hendriks, M.A.N.H. Identification of the mechamical behawiour of golidil materiats. Phal thesis, Eindhoven University of Technology, Eindhoven., The Netherlands, 1991.

Hickey, D.S. Hukins, D.W.L. Effect of methods of preservation on the arrangement of collagen fibrils in connective tissue matrices: an X-ray diffraction study on andus fibrostis. Connective "Tisue Resedarch, $6: 223-228,1979$.

Holmes, M.H., Lai, W.M., Mow, V.C. Singular pertubation analysis of the nonlinear, Mow-dependent compressive stress relaxation behaviour of articular cartilage. ASME J. Bionnech. Eng., 107:206-218, 1985. 
Hotmes, M.H. Fince defornation of soft tissue: analysis of a mixture model in uni-axial compression. A ME W. Bumech. Eng., 108:372-381, 1986.

Johnstone, A., Urban, J.P.G., Roberts, J., Menage, J. The fluid contents of the human interwertebral disc. Spinte, $13: 412-416,1992$.

Kwan "M.K., Hacker, S.A."Woo, S. L-Y, Wayne, J.S. The effect of storage on the biomechanical behaviour of articular cartilage - a large strain study. Journal of Bomechanical enginering, 114:149-153, 1992.

Lai, W.M., How, I.S., Mow, V.C. A triphasic theory for the swelling and deformation behaviours of articular cartilige. Joumall of Biomechanical Engineering, 113:245-258, 1991.

Myers, E.R., Lai, W.M., Mow, V.C. A continum theory and an experiment for the ion-induced swelling behaviour of articular cartilage. Journal of Biomechanical Engineering, 106:151-158, 1984.

Mow, V.C., Kuei, S.C., Lai, W.M., Armstrong, C.G. Biphasic ereep and stress relaxation of articular cartiage in compression: theory and experiments. Joumal of Biomechanical Engineering, 102:73-84, 1980.

Ohshima, H, Tsuji, H. Hirano, N. Ishihara, H., Katoh, Y., Yamada, H. Water diffusion pathway, swelling pressure and biomechanical properties of the intervertebral disc during compression load. Spine, $14: 1234-1244,1989$.

Panjabi, M.M., Krag, M., Summers, D., Videman, T. Biomechanical tme-tolerance of fresh cadaveric human spine specimens. Journal of Orthopaedic Research, 3:292-300, 1985.

Sinjiders, H., Hayghe, J., Willems, P., Drost, M., Janssen, I., Huson, A. A mixture approach to the meehamics of the human intervertebral disc. in: Mechanics of swelling: from days to living cells and tissues, ed. T.D. Karnilis, 1992.

Urban, J.P.G., Maroudtws, A., Bayliss, M.T., Dillon, I, Swelling pressures of proteoglycans at the concentrations found in cartiliginous tissues. Biorheology, 16:447-464, 1979',

Urban, J., Maroudas; A. Measurement of swelling pressure and fluid flow in the intervertebral disc with respect to ereep. Proc. Inst. Mech.. Engr., C132/80,63-69, 1980.

Unhan, J.P.G., MoMullim, I.F. Swelling pressure of the intervertebral dise: influence of proteoglycan und collagen contents. Biorheology, 22:145-157, 1985 .

Uibun, J.P.C., McMullin, J,F. Swelling pressure of the lumbar intervertebral dises: influence of age, spinal llevel, composition and degeneration. Spine, 13:179-187, 1988.

Vos, M.A., Gorgels, A. Leunissen, $J$, Wellens H. Flumarizine allows differentiation betwen thechanisms of arbythmas in the intact heart. Ciroulation, $81: 343-349,1990$.

Vos, M.A., Gorgels, A., de Wit, B., Drenth, J., van Deursen, R., Leunissen, J., Wellens, H. Premature excape bests: a model for triggered activity in the intact heart? Cimalation, 82:213-224, 1990.

Woltring, H.\%. A fortran package for generalized, cross-validatory spline smoothing and differentiation. Adr. Eng. Software, 8:104-107, 1986. 


\section{OSMOTIC PRESTRESSING OF A SPINAL MOTION SEGMENT}

The mechanical behaviour in axial compresision of a human lumbar motion segment (MS) without the facet joints (posterior elements) is modelled using two porous medium descriptions, one including, the other excluding Donnan osmosis. Donnan osmosis is shown to affect the anulus fibre and ground matrix stresses and the fluid flow through the intervertebral disc. Hence, models not including the osmotic effect appear to be inadequate with respect to the prediction of these quantities. In an axial compression simulation of the motion segment major differences occur in axial displacement (after $10 \mathrm{~h}$ biphasic $16 \%$ more than triphasic), expelled fluid volume versus time (after 10 h triphasic $28 \%$ less than biphasic), fluid pressure (at the base at the inner anulus after $50 \mathrm{~s}$ triphasic $26 \%$ higher than biphasic), and fibre stress (at the base at the anulus edge after $50 \mathrm{~s}$ triphasic $60 \%$ higher than biphasic). Although the triphasic simulation mainkains a higher fluid pressure, less water is expelled. 


\subsection{Introduction}

The human lumbar spine constitutes a mechanical unit that allows for flexion, extension and rotation, while it supports the body. The basic unit of the spine is the motion segment (MS) which consists of 2 vertebrae that enclose the intervertebral disc (IVD). Intervertebral disc tissue consists of a collagen and elastin fibre network embedded in a hydrated proteoglycan (PG) matrix. Small nutrients and ions are dissolved within the tissue. Because of the entanglement of the PG and the fibre network only the interstitial fluid and the small ions can flow. The PG are ionized and because they are relatively stagnant, osmotic effects are important (Urban and Maroudas, 1979).

Mechanical models of the IVD are described in terms of the theory of mixtures. In the biphasic mixture model the solid and fluid phases are regarded as two kinematically distinct continua. The biphasic model has been used as a basis for finite element modelling of the IVD (Simon et al., 1985). When, however, the osmotic effect plays an important role, it must be accounted for as a third phase: the ionic phase (Lai et al., 1991, Snijders ef al., 1992). As the osmotic force is deformation dependent, it cannot be accounted for in the biphasic model using a constant term. To demonstrate the differences between the bi- and triphasic approaches, we compared the models in an axial compression simulation of a motion segment.

\subsection{Material and methods}

\subsubsection{Theory}

Deformation of the tissue can be achieved either by mechanical or chemical loading. The overall response is a result of the (1) diffusion of mobile ions, (2) the large deformation of the fibre network and the ground substance with relative fluid flow, and (3) osmosis. In order to describe this complex behaviour we use the theory of mixtures (Bowen, 1980, Müller, 1985). Starting with the kinematic relationships and the general balance laws, specific assumptions for intervertebral disc tisste are incorporated and constitutive restrictions based on the entropy principle are derived (Snijders et al., 1992). The resulting equations are: mass balance (continuity) of the mixture, momentum balance of the mixture and the diffusion equation of the ions. In this study no ion diffusion is used, but simply an instantaneous ion distribution based on Donnan equilibrium for large negatively charged molecules (PG) and small univalent ions. This is admissible because of the constant external ion concentration. Due to the solid deformation ions will still move out of the tissue, but this migration is taken up largely by the convection with water. Hence, diffusion only plays an important role in the local redistribution of ions. The equations are given below: 


$$
\begin{array}{ll}
\text { momentum } & : \vec{\nabla} \circ \mathcal{G}-\vec{\nabla} p=\overrightarrow{0} \\
\text { continuity } & : \vec{\nabla} \circ \vec{v}^{s}-\vec{\nabla} \circ[K \circ \vec{\nabla}(p-\varpi)]=0 \\
\text { ion distribution } & : 2 c=-c^{p g}+\sqrt{\left(c^{p g}\right)^{2}+4 c^{2}} \\
\text { osmotic retension } & : \varpi=\phi R T\left(2 c+c^{p g}\right)
\end{array}
$$

with $\quad$ the solid Cauchy stress tensor $p$ the hydrodynamic pressure $\checkmark$ the velocity of the solid. $\underline{K}$ the permeability tensor

$c$ the anion concentration $\bar{c} c$ the external salt concentration $\mathrm{c}^{\mathrm{pg}}$ the proteoglycan concentration $\phi$ the osmotic coefficient

The primary unknowns are the displacement of the solid $\vec{u}$, the fuud pressure $p$ and the ion concentration $c$. The equations above, combined with the appropriate material behaviour, initial and boundary conditions complete the description of the mechanical behaviour of intervertebral disc tissue. To solve this problem the Finite Elentent Method is used (Snijders et al, in press). A Total Lagrange (Newton-Raphson iteration scheme, Houbolt third order time integration scheme) formulation based on the Galerkin method is implemented in the commercial FE-package DIANA (DIANA Analysis B. W., Delft, the Netherlands). For the numerical studies iso-parametric elements of the serendipity family are used. Two dimensional plane strain, three dimensional and axisymmetric elements have been developed. The interpolation functions are the same for displacements, pressures and concentrations and can be either linear or parabolic.

\subsubsection{Geometry}

For an axial compression load less than $1000 \mathrm{~N}$ the facet joints do not contribute significantly to the load transmission (Adams and Hutton, 1980). As the load which is used in the calculations is $650 \mathrm{~N}$ maximum the posterior elements are neglected. For the sake of simplicity the remaining disc and vertebra are approximated by an axi-symmetrical geometry. In figure 6.1 the dimensions of the modelled motion segment of the lower lumbar spine (L4-L5) are given. For the disc and vertebra the gross dimensions are in agreement with the anatomical data of Aharinejad et al. (1990), for the endplate Robert et al. (1989) is used. The chosen geometry and its dimensions are comparable with ShiraziAdl (1989).

Six different tissue types are modelled: nucleus pulposus (NP), anulus fibrosus (AF), cartilaginous endplate (CEP), bony endplate (BEP), cancellous bone (CAB), and cortical bone (COB). The transverse areas of the nucleus and the anulus are equal. The outer anulus area equals also the latter areas. Both $z=0$ and the top are planes of symmetry. In figure 6.1 the different areas and materials are depicted. 


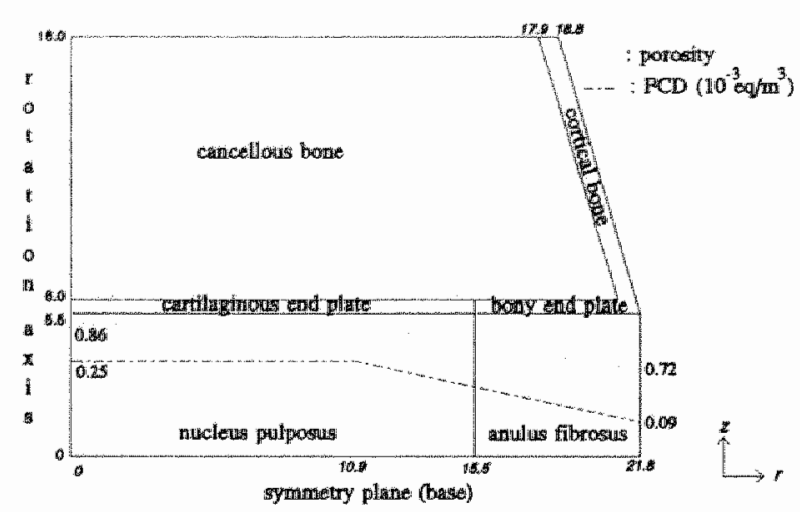

figure 6.1 The geometry and materials; the porosity (volume fraction of water) and the fuxed charge density as a function of radial distance are given. The dimensions (italic numbers) are in $\mathrm{mm}$.

\subsubsection{Material properties}

The triphasic material behaviour of the tissue involves the stress-strain relation, the permeability, the porosity, the proteoglycan concentration or fixed charge density (FCD), and the osmotic coefficient. Only the 'soft' tissues are supposed to be osmotically active. The radial profile of the FCD across the nucleus and the anulus is given in figure 6.1 (Urban and Maroudas, 1979). In the axial direction a homogenous distribution is assumed. The radial profile of the FCD in the CEP equals the one of the nucleus (Roberts et al., 1989). In the axial direction of the CEP the FCD decreases linearly to the value in the CAB: $0.001 * 10^{+3} \mathrm{eq} / \mathrm{m}^{3}$. The porosity profile of the soft tissues has the same shape as the FCD profile (Urban and Maroudas, 1979). For the cancellous bone a value of 0.5 is used. As the cortical bone and the bony endplate are dense materials a porosity of 0.01 is used. The osmotic coefficient is the same for all tissues and equals $2.1 \mathrm{kPa} /\left(\mathrm{m}^{3} \mathrm{eq}\right)$.

For all tissue types a linear relationship between the second Piola-Kirchhoff stress and the Green-Lagrange strain is assumed. The bi- and triphasic material properties are taken to be identical. The nucleus and the bony parts are isotropic. The Young's moduli and the Poisson's ratios are listed in table 1. The anulus fibrosus and the cartilaginous endplate are composed of a ground substance with aligned imbedded collagen fibres. Both tissues are viewed upon as a homogenous mixture of matrix and fibres. Due to the orientation of the fibres the mixture has direction dependent properties. A perfect bond between the fibres and the matrix is assumed. The properties of the mixture are calculated according to the rules given by Hashin and Rosen (1.964). The initial parameters needed are: the Young's modulus, Poisson's ratio, and the volume fraction of the fibres, the Young's modulus and the Poisson's ratio of the matrix. The Young's modulus and volume fraction of the fibres increase almost linearly in radial direction across the anulus. The volume fraction increases from $12.5 \%$ to 20.9\% (Brickley-Parsons and Glimcher, 1983), the Young's modulus from $225 \mathrm{MPa}$ to $595 \mathrm{MPa}$ (Marchand and Ahmed, 1989). For the CEP values of $35 \%$ and $200 \mathrm{MPa}$ are used. We found no experimental data for the 
Poisson's ratio of the fibres and assume a value of zero. A sensitivity analysis for fibre Poisson's ratios varying from 0 to 0.4 shows the following changes in the nixture (matrix + fibre) properties after rotation to model axes: maximal $0.3 \%$ in the Young"s moduli and maximal $15 \%$ in the Poisson's ratios. The material constants are listed in table 6.1.

\begin{tabular}{|c|c|c|c|c|c|}
\hline & $\begin{array}{l}\text { Young } \\
\text { modulus }\end{array}$ & $\begin{array}{l}\text { Poisson"s ratio } \\
\text { fibre \% }\end{array}$ & Permeability & Porosity & $\mathrm{FCD}^{*}$ \\
\hline & $\mathrm{MPa}$ & {$[-] / \%$} & $10^{-16} \mathrm{~m}^{4} / \mathrm{Ns}$ & {$[-]$} & $10^{+3} \mathrm{eq} / \mathrm{m}^{3}$ \\
\hline $\mathrm{NP}$ & 1.5 & 0.11 & 9.0 & figure 6.1 & figure 6.1 \\
\hline $\begin{array}{l}\text { AF matrix } \\
\text { inner fibres } \\
\text { outer fibres }\end{array}$ & $\begin{array}{l}2.5 \\
225 \\
595\end{array}$ & $\begin{array}{l}0.1 /- \\
0.0 / 12.5 \\
0.0 / 20.9\end{array}$ & 9.0 & figure 6.1 & higure 6.1 \\
\hline $\begin{array}{l}\text { CEP matrix } \\
\text { fibres }\end{array}$ & $\begin{array}{l}2.5 \\
200\end{array}$ & $\begin{array}{l}0.1 / 1 \\
0.0 / 35.0\end{array}$ & 27.0 & figure 6.1 & figure 6.1 \\
\hline $\mathrm{BEP}$ & 50 & $0.3 /$ & 0.01 & 0.01 & 0.001 \\
\hline $\mathrm{CAB}$ & 100 & $0.3 / \%$ & 1000.0 & 0.01 & 0.001 \\
\hline $\mathrm{COB}$ & 10000 & $0.3 /-$ & 0.01 & 0.5 & 0.001 \\
\hline
\end{tabular}

table 6.1 Material constants for a human lumbar motion segment. The F(ixed) $\mathrm{C}$ (harge) $\mathrm{D}$ (ensity) is defined as the number of equivalent (charge) moles per $\mathrm{m}^{3} \mathrm{H}_{2} \mathrm{O}$. $\because$ the FCD is used in the triphasic model only.

The fibres, arranged in lamellae, incline with respect to the transverse plane. The angle changes from $+\alpha$ to $-\alpha$ from one layer to the other. For $\alpha$ a value of 30 degrees is used for the anulus fibres, while an angle of 10 degrees is used for the cartilaginous endplate. With respect to a coordinate system aligned in the fibre direction a transverse isotropic stress-strain relation $\operatorname{SE}(\alpha)$ is obtained. Rotating this relationship to the global coordinate system yields a stress-strain relation with coupling between shear stresses and normal strains. By combining the contribution of a $+\alpha$ and a $-\alpha$ lamella according to SE $=1 / 2(\operatorname{SE}(\alpha)+\operatorname{SE}(-\alpha))$ the coupling terms vanish. The resulting stress-strain relation is orthotropic in the global coordinate system.

\subsubsection{Boundary conditions}

The initial heights of the bi- and triphasic MS are taken to be identical. Because the triphasic MS has a swelling tendency, a preload has to be applied in order to keep its height identical to the biphasic height. The magnitude of this load is calculated by fixing the MS to the predetermined height, letting it swell until equilibrium is reached and calculating the axial force that is required to keep it in place. In addition to this preload the actual load is applied. In the biphasic case no preload is required and the simulation starts with the immediate load application. The actual test load is $650 \mathrm{~N}$ for both the models. Both the base $(z=0 \mathrm{~mm})$ and the top $(z=16.0 \mathrm{~mm})$ are planes of symmetry. Due to axi-symmetry the displacements at $r=0$ equal zero in the $r$-direction. At the base the displacements in the z-direction equal zero. Above the nucleus lymphatic activity yields 
runs from $0.05 \mathrm{MPa}$ (outermost anulus sheet) to $0.16 \mathrm{MPa}$ (innermost anulus sheet). The naximum pressutes in the nucleus are:

$\begin{array}{lll} & \text { biphasic } & \text { triphasic } \\ \text { initial } & 0 \mathrm{MPa} & 0.188 \mathrm{MPa} \\ \text { after } 10 \mathrm{~s} & 0.480 \mathrm{MPa} & 0.663 \mathrm{MPa} \\ \text { after } 10 \mathrm{~h} & 0.008 \mathrm{MPa} & 0.261 \mathrm{MPa}\end{array}$
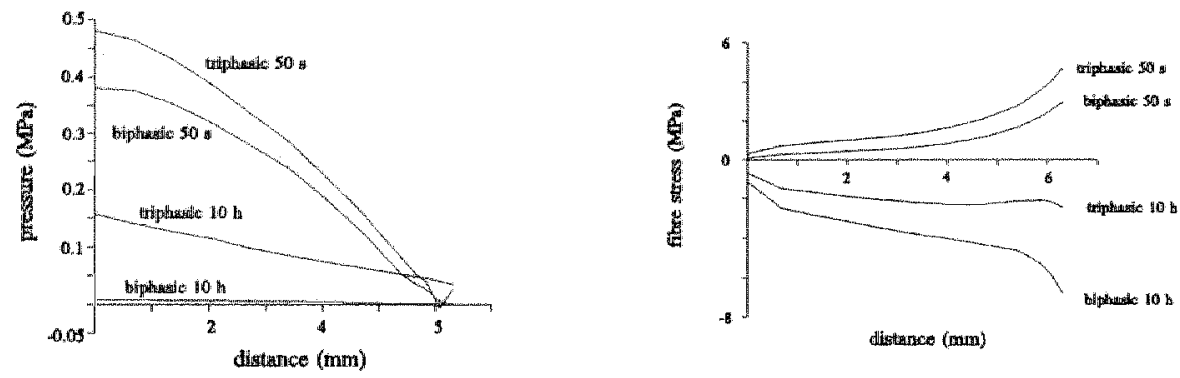

figure 6.4 Fluid pressure (left) and 2nd Piola Kirchhoff stress (right) along the anulus base

\subsubsection{Fibre stress}

The fibre stress curves along the anulus base for different times are shown in figure 6.5 . Initially, (after 50 s) the fibre stresses are largest. They decrease in time and become negative ultimately. The triphasic stresses are higher than the biphasic stresses. After $50 \mathrm{~s}$ the triphasic stress at the base at the anulus edge is $60 \%$ higher than the biphasic stress (4.8 $\mathrm{MPa}$ versus $3 \mathrm{MPa})$.

Summarizing we find that in ax axial compression simulation of the motion segment major differences occur in axial displacement (biphasic $16 \%$ more than triphasic), expelled fluid volume versus time (after $10 \mathrm{~h}$ triphasic $28 \%$ less), fluid pressure (at the base at the inner anulus after $50 \mathrm{~s}$ triphasic $26 \%$ higher than biphasic), and fibre stress (at the base at the anulus edge after $50 \mathrm{~s}$ triphasic $60 \%$ higher than biphasic). Although the triphasic simulation maintains a higher fluid pressure, less water is expelled.

\subsection{Discussion}

This study is to our knowledge the first to present a triphasic finite element model of the intervertebral disc. The comparison of biphasic and triphasic simulations shows that (1) 
the triphasic model holds on to more water although the triphasic fuid pressure gradients are larger and (2) the anulus tensile fibre stresses are larger in the triphasic computation compared to the biphasic computation. The triphasic simulation loses less height (3) and reaches equilibrium earlier (4).

In comparing the two models it is important to be aware of some fundamental differences between them. In both models it is possible for the structures to attract water. The mechanisms by which this occurs are completely different. In the biphasic model fluid can be imbibed because of volume change of the tissue. In the triphasic case the atraction of fluid molecules has its origin in the osmotic effect and the volume change of the tissue. Because the osmotic force is deformation dependent through the dependency on the $\mathrm{FCD}$, the biphasic model cannot be simply adjusted by adding a simple term to the biphasic stiffness to compensate for the osmotic stiffness. A triphasic model is needed.

Berkson et al. (1979) measured fluid pressures in the nucleus in axial compression experiments on motion segments with excised face joints. They found that the nucleus pressure was 1.22 (compression load $400 \mathrm{~N}$ ) to 1.99 (compression load $100 \mathrm{~N}$ ) times the outer applied load per unit surface of the IVD. The values should be compared shortly after application of the load $(<15 \mathrm{~min})$ at which time the measurements in vitro have been performed. For the triphasic simulation this factor $(\mathrm{P})$ is 1.87 initially (with the preload of $150 \mathrm{~N}$ ). In the loading stage shortly (10 s) after application of the load it is 1.24 , and after $10 \mathrm{~h}$ it equals 1.10 . In the biphasic experiment the values after $10 \mathrm{~s}$ and $10 \mathrm{~h}$ are, respectively, 0.49 and 0.018 . There are three important conclusions to be drawn from these data: (1) The factor $P$ depends on the external load (experiment + simulation), (2) $P$ is dependent on time (simulation), and (3) $P$ is greater than one in the triphasic case and in the experiment, whilst it is smaller than one for the biphasic computation. This means that the biphasic maximum nucleus pressure is smaller than the outer load per unit area of IVD. The reverse is true for the triphasic computation and the experiments. One should bear in mind that the values of $\mathrm{P}$ reported by Berkson et al. are based on in vitro measurements and our simulations are in viwo simulations or in vitro experiment in physiological saline.

Although the triphasic fluid pressure is higher (figure 6.4), less water is expelled (figure 6.3b): the triphasic material holds on to more water at higher pressures. This effect can be understood when we consider that the driving force for fluid flow is the combined effect of concentration gradients and pressure gradients (6.2.1).

The triphasic fibre stress is seen to be higher at all points in time, as is shown in figure 6.5 for $t=50 \mathrm{~s}$ and $t=10 \mathrm{~h}$. The triphasic model would predict earlier breakage of fibres than would the biphasic model. In time, the fibre stresses decrease uniformly. This means that fibre breakage in this modelling situation is most likely to occur directly after application of the load. It should be noted that the fibre angle also determines the value of the stress (through rotation of the stresses in the model axes), while the measured values show a wide range (variation by as much as $35^{\circ}$ ), even in one individual fibre sheet (Marchand and Almed, 1990). By choosing a smaller angle (than 30\%) the stresses will increase. Tensile Cauchy stress at failure of anulus specimens cut along the fibre direction was found to be $8.8 \pm 1.1 \mathrm{MPa}(n=18$; Galante, 1967).

The comparison of radial deformation with experimental data is complicated because of the influence of the preload (Panjabi and $\mathrm{Krag}, 1977$ ), and the time dependence of the axial deformation (figure 6.2). Panjabi and Krag found significant differences in radial 
deformation using 3 different preloads: 0,400 and $1000 \mathrm{~N}$ and very small additional: loads: up to $150 \mathrm{~N}$. The axial settlement at a load of $150 \mathrm{~N}$ with the different preloads lay in the range of -0.01 to $-0.02 \mathrm{~mm}$. They also concluded that the effect of preload may be different for different physiological loads. Their findings make it difficult to compare the numerical results with $150 \mathrm{~N}$ preload and $650 \mathrm{~N}$ additional load with experimental results without preload. Hirsch and Nachemson (1954) found a mean axial displacement of -0.75 $\mathrm{mm}(\mathrm{n}=94 ;-1.5 \mathrm{~mm}$ for the whole motion segment; our axiall displacement data are based on half of the MS) with a load of $1000 \mathrm{~N}$. This is smaller than our predicted values after $10 \mathrm{~h}$. However, they measured after a relatively short time (minutes) after the application of the load, while we find at that point in time significant creep response in our simulation. Therefore, orders of magnitudes only can be compared.

Berkson et al. (1979) measured no-load intradiscal pressures and found a mean value of $0.076 \mathrm{MPa}(\mathrm{n}=42)$ with the posterior elements (facet joints) destroyed. In unloaded biphasic material in which the pores are in open contact with a fluid environment, the pore pressure necessarily goes to the pressure of the environment. We did not model the unloaded situation, but in the preload equilibrium situation the nucleus pressure ranges from $0.11 \mathrm{MPa}$ to $0.19 \mathrm{MPa}$. These values may be comparable with the experimental data of Berkson et al., keeping in mind that the $150 \mathrm{~N}$ preload has a $\mathrm{P}$ factor of 1.9 for the pressure of $0.19 \mathrm{MPa}$ and 1.1 for $0.11 \mathrm{MPa}$.

\subsection{References}

Adams, M.A., Hutton, W. C. The effect of posture on the role of the apophysial joints in resisting intervertebral compressive forces. J. Bone Joint Surg. [Br], 62B:358-362, 1980

Aluarinejad, S., Bertagnoli, R., Wicke, K., Firbas, W., Schneider, B. Momphometric analysis of vertebrae and intervertebral discs as a basis of disc replacement. Am. J. Anat., 189:69:76, 1990.

Berkson, M.H., Nachemson, A., Schultz, A.B. Mechanical properties of human lumbar spine motion sogments-Part 2: responses in compression and shear; influence of gross morphology. Journal of Biomechanical Enginesering, $101: 53-57,1979$

Bowen, R.M. Incompresibible porows media nodels by use of the theory of mixtures. Int. J. Eng. Sci. Vol $18,1129-1148,1980$

Brickley-Parsons, D., Glimoher. M.J. Is the chenistry of eollagen in intervertebral discs an expression of Wolff law? A study of the human lumbar spine. Spine, $9,2: 148-163,1984$.

Gallante, J.O. Tensile properties of the human lumbar anulus fibrosis. Acta Orthopedica Scandinavica Suppl. $100: 1: 5-91,1967$

Hashin, 2., Rosen, B.W. The elastic moduli of fber-reinfored materials. J. Appl. Mech., 223-230, 1964

Mirsh, C. Wachemson, A. A new observation on the mechanical behaviour of llumbar discs. Acta Orthop. Scan., $23: 254-283,1954$

Lai, W.M., How, J.S., Mow, V.C. A triphasic theory for the swelling and deformation behaviors of articular cartilage. J. of Biomech. Engng., 113:245-258, 1991 
Marchand, F., Ahmed, A.M. Mechanical properties and failure mechanism of the constituant cornaponents of anulus fibrosus. Proc. 10th Annu. CBS, Montreal, Quebec, 1989.

Müller, I. Thermodynamics. Pitman Advanced Publishing Program, Boston, 1-202, 1985

Panjabi, M.M, Krag, M.H. Effects of prelload on load displacement curves of the lumbar spine. Orthopedic Clinics of North America, 8:181-192, 1977

Roberts, S., Menage, J., Urban, J.P.G. Biochemical and structural properties of the cartilage end-plate and its relation to the intervertebral disc. Spime, 14:166-174, 1989 .

Shirazi-Adl, A. On the fitbre composite material models of disc anulus-comparison of predicted stresses. 3 . Biomech., 22:357-365, 1989.

Simon, B.R., Wu, J.S.S., Carltom, M.W., Evans, J.H., Kazarian, L.E. Structural models for human spinal motion segments based on a poroelastic view of the intervertebral disk. J. Biomech. Engng. 107,327-335, 1985.

Snijders, H., Huyghe, J., Willems, P., Drost, M., Janssen., J., Huson, A. A mixture approach to the mechanics of the human intervertebral disc. in: Mechanics of swelling: from clays to living cells and tissues, ed. T.D. Karalis, 1992.

Snijders, H., Huyghe, J.M., Drost, M.R., Willems, P., Janssen, J.D., Huson A. Triphasic finite element model for intervertebral dise tissue. in: Computer Methods in Biomechanics and Biomedical Engineering Symposium, Middleton, J., Pande, G.N., Williams, K.R. (eds), in press

Urban, J., Maroudas, A. The measurement of fixed charge density in the intervertebral disc. Blochimica et Biophysica Acta, 586:166-18, 1979. 



\section{SUMMARY CONCLUSIONS AND RECOMMENDATIONS}

In section 7.1 the study presented in this thesis is summarized. The conclusions (7.2) are grouped in three categories: the presented theory, the experimental method and data, and the numerical simulations. In section 7.3 the recommendations are given, grouped in the same categories as the conclusions. 


\subsection{Summary}

In the industrial world low back pain is one of the important causes of absence and incapacity for work. Besides the financial loss there is the personal loss of quality of life. Epidemiological research relates low back pain to the mechanical loading of the spinal column. During lifetime the biochemical and structural composition of the intervertebral disc changes, often referred to as degeneration. In order to analyze the chemo-mechanical interaction a theoretical model is needed, which lakes into account the structural and chemical composition of intervertebral disc tissue.

The presented framework in this thesis is based on the theory of mixtures. Starting with the kinematic relationships and the general balance laws constitutive restrictions based on the entropy principle are derived. The constitutive restrictions may depend on the local chemical composition and the local deformation. The model includes: (1) large deformations of the solid component, (2) relative fluid flow caused by gradients in the chemical potential of the fluid and diffusible ions, (3) non-ideal Donnan osmosis and electro neutrality, (4) convection-diffusion of the small ions. The model is capable of describing the equilibrium results in the physiological range of a confined compression test reasonably well. It also shows that the apparent dependence of the elastic constants on the local ion concentration originates from the Donnan osmotic pressure.

The equations describing the chemo-mechanical behaviour of intervertebral disc tissue are three coupled partial differential equations in which geometric and physical nonlinearities occur. To solve them for an arbitrary geometry and arbitrary boundary conditions we use the Finite Element Method. A Total Lagrange (Newton-Raphson iteration scheme, Houbolt third order time integration scheme) formulation based on the Galerkin method is implemented in the commercial FE-package DIANA (DIANA Analysis B.V., Delft, the Netherlands). For the numerical studies iso-parametric ellements of the serendipity family are used. Two dimensional (plane strain and plane stress), three dimensional and axisymmetric elements have been developed. The interpolation functions are the same for displacements, pressures and concentrations and can be either linear or parabolic.

In order to test whether the proposed model is realistic one dimensional confined compression experiments on canine samples are performed. The samples are taken from the canine anulus fibrosus. Two orientations of the specimen with respect to the spinal axis are used: perpendicular and parallel. "The loading protocol consists of a combination of mechanical and chenical loading. The experimental data is successfully fitted to the proposed model. Triphasic evaluation of obtained data shows that swelling behaviour is caused by Donnan osmosis. The experimental data does call for the introduction of salt dependent elastic constant and so-called chemical expansion stress. The aggregate modulus and the permeability are deformation dependent. The permeability and aggregate modulus differ significantly for the axial and radial specimen.

The consequences of modelling a motion segment bi- or triphasically is studied by analysing the mechanical behaviour in axial compression of a human lumbar motion segment without the facet joints (posterior elements). The calculated deformation and fluid 
flow versus time agree reasonably well with experimental observations reported in the literature. Donnan osmosis is shown to affect the anulus fibre and ground matrix stresses and the fluid flow through the intervertebral disc.

In conclusion, the present model for the chemo-mechanical behaviour of intervertebral disc tissue is capable of describing the response of the tissue to both a chemical and/or mechanical load.

\subsection{Conclusions}

The mechanical function of the different components in intervertebral disc tissue has been investigated. The role of swelling and/or osmotic effects has been emphasized. Based on generally accepted thermodynamic principles the chemo-mechanical behaviour of the tissue is derived. Despite the large number of simplifications and suppositions, the presented frame work is capable of describing the experimentally observed deformation of isolated specimen and motion segments under changing chemical and mechanical loads.

\subsubsection{Conclusions concerning the presented theory}

- A theoretical framework based on the theory of mixtures which takes into account the biochemical and structural composition of the tissue has been derived:

finite deformation of the groundsubstance and fibre network

relative fluid flow

osmosis

diffusion of small ions into a charged matrix.

- Compressive forces are counter balanced by forces due to deformation of the ground substance and the osmotic pressure due to gradients in the proteoglycan concentration. During the deformation process the load is transferred from the fluid component to the solid component. In contrast with a biphasic approach the fluid ultimately bears a part of the load due to the osmotic pressure. As fluid is expelled from the tissue and the proteoglycan concentration increases, the contribution of the osmotic pressure to the load transmission increases.

- Within the physiological range the presented framework is qualitatively in agreement with the confined compression equilibrium response of a chemically and/or mechanically loaded specimen as measured by Urban and Maroudas (1980).

- The model predictions for non physiological hydrations can not be improved by adding a chemical expansion stress as proposed by Mow et al. (199)) and Lai et al. (1991) to the Donnan osmotic pressure. Introducing a deformation and/or ion concentration cependent stress-strain relation (Mow and Schoonbeck, 1984, Eisenberg and Grodzinsky, 1985, 1987, Myers et al., 1984) also gives no improvement.

- The apparent dependence of the elastic constants of the stress-strain relation on the local salt concentration (Mow and Schoonbeck, 1984, Eisenberg and Grodzinsky, 1985, Myers et al., 1984) originates from the Donnan osmotic pressure. 


\subsubsection{Conclusions concerning the experimental method and data}

- The specimen preparation, the experimental set-up and the experimental protocol yields reproducible results.

- The evaluated material parameters lie within the physiological range except the diffusion coefficient of $\mathrm{NaCl}$.

- Triphasic evaluation of the data shows that both the stress-strain relation and the permeability of the canine anulus fibrosus are deformation dependent, even for linear strains less then $10 \%$. Specimen to specimen variability of the aggregate modulus and the permeability are correlated with each other.

- The swelling behaviour is caused by Donnan osmosis. The experimental results show no need for introducing a chemical expansion stress as has been done by Lai et al. (1991).

- While in contact with a $0.2 \mathrm{M} \mathrm{NaCl}$ solution, and increasing the external load from $0.08 \mathrm{MPa}$ to $0.2 \mathrm{MPa}$, the osmotic pressure contributes for only $15 \%$ to the mechanical load, $85 \%$ is counterbalanced by tissue deformation.

- The transport coefficient and aggregate modulus, as roughly evaluated by applying the linear biphasic theory to the 1-D consolidation data, differ significantly for the axial and radial specimen.

- The deformation averaged triphasic material properties differ from the deformation averaged biphasic material properties. Therefore, use of the biphasic theory for quantification of the permeability and aggregate modulus from consolidation data should be avoided, particular when large strains are involved.

\subsubsection{Conclusions concerning the numerical simulations}

- Geometrically (linear strain of $25 \%$ ) and physically (deformation dependent aggregate modulus and the permeability) non-linear biphasic simulation of the confined compression experiment on articular cartilage (Holmes, 1986) shows that:

1: the response immediately after load application is still linear in the square root of time.

2: the shift to longer consolidation times due to the deformation dependent permeabiinty is almost fully compensated by the shift to smaller consolidation times due to the stiffening stress-strain relation.

- Geometrically and physically non-linear biphasic versus triphasic simulation of the axial compression of a motion segment shows that:

1: the triphasic model holds on to more water although the triphasic fluid pressure gradients are larger.

2: the anulus tensile fibre stress are higher in the triphasic simulation. Therefore, a biphasic model overestimates the load bearing capacity at a given fibre yield stress.

3: the maximum stress is reached immediately after load application.

3: the triphasic motion segment reaches equilibrium earlier.

4: the nucleus pressure / external load ratio for the triphasic simulation is in agreement with the results of Berkson et al. (1979). The biphasic is not.

5: the axial displacement of the vertebral bone and the radial bulge are qualitatively in agreement with experimental results for both the triphasic and biphasic simulations. 


\subsection{Recommendations}

\subsubsection{Recommendations concerning the presented theory}

- Different experimental set-ups (free swelling or multi axial deformation) should be used for further validation of the theory. The presented constitutive framework should be improved based on the experimental data.

- The applicability of the theory to other soft biological tissues, such as cartilage, skin and subcutis, muscle and cornea, and to technical materials, such as hydrogels, shales, clay should be verified.

\subsubsection{Recommendations concerning the experimental method}

- Experimental validation of the presented theoretical framework is cumbersome due to the variability of the properties of biological tissue. Using an artificial substance with the same chemical and mechanical characteristics will overcome the limitation of biological tissue.

- The endplate is believed to play a crucial role in the nutrition, fluid flow and failure (auto-immune response) of the intervertebral disc. The triphasic and failure characterisation of the endplate should therefore be investigated.

- Nucleus and endplate samples can be obtained using a corer in stead of the turning procedure.

- The experimental protocol can be improved by:

1: using a analogue low pass filter to improve the signal-noise ratio

2: using inhibitors to decrease the autolysis of the tissue

3: determination of the chemical composition of the sample, e.g. the proteoglycan, collagen and fluid concentration

4: direct measurement of the diffusion coefficient using tracer ions

- In order to analyze the load transmission in the human spine, human experimental triphasic data should be obtained, both for isolated specimen and complete motion segments.

\subsubsection{Recommendations concerning the numerical simulations}

- Complex loading situations, using a three dimensional geometry will render valuable information about the load transmission in the motion segment. The influence of the orientation of the anulus and endplate fibres, or with appropriate damage models the development of a slipped disc can be studied.

- Clinical treatments such as chemo-nucleuses can be evaluated.

\subsection{References}

Berkson, M.H., Nachemson, A., Schultz, A.B. Mechanical properties of humam lumbar spine motion segments-Part 2: responses in compression and shear; influence of gross morphology. Journal of Biomechanical Engineering, 101:53 57, 1979

Eisenberg, S.R., Grodzinsky, A.J. Swelling of articular cartilage and other connective tisunes: electrom mechanochemical forces. J. Orthop. Res., 3:148-159, 1985 . 
Eisenberg, S.R., Grodzinsky, A.J. The kinetics of chemically induced nonequilibrium swelling of articular cartilage and comeal stroma. J Biomech. Engng., 109:79-89, 1987.

Holmes, M.H., Lai, W.M., Mow, V.C. Singular pertubation analysis of the nonlinear, flow-dependent compressive stress relaxation behaviour of articular cartilage. ASME J. Bionech. Eng., 107:206-218, 1985.

Holmes, M.H. Finite deformation of soft tissue: analysis of a mixture model in uni-axial compression. ASME 1. Biomech. Eng. 108:372-381, 1986.

Lai, W.M., Hou, J.S., Mow, V.C. A triphasic theory for the swelling and deformation behaviours of articular cartilage. Journal of Biomechanical Engineering, 113:245-258, 1991.

Myers, E.R., Lai, W.M., Mow, V.C. A continuum theory and an experiment for the ion-induced swelling behaviour of articular cartillage. Journal of Biomechanical Engineering, 106:151-158, 1984.

Mow, V.C., Lai, W.M., Hou, J.S. Triphasic theory for swelling properties of hydrated charged soft biological tissues. Appl. Mech. Rev., 43:134-141, 1990.

Urban, J., Maroudas, A. Measurement of swelling pressure and fluid flow in the intervertebral disc writh reference to creep. Proc. Inst. Mech. Eng., C132/80:63-69, 1980. 


\section{Curriculum Vitae}

20-5-1959

1971-1978

1978-1986

$1984-1985$

1986-1987

1987-1992

1992-heden geboren te Heerien

Atheneum B, Bernardinus College, Heerlen

Werktuigbouwkunde,

Technische Universiteit Eindhoven

Laboratorium voor Experimentele Orthopedie,

Katholieke Universiteit Nijmegen

DSM Research, Geleen

Rijksuniversiteit Limburg, vakgroep Bewegingswetenschappen

TNO-Bouw, Rijswijk 


\section{Samenvatting}

Lage rugpinn is ew van de belangrijkste redenen voor werkwerzuim en arbeidsongeschiktheid. Naast de financiële gevolgen is er sprake van een verlies van kwaliteit wan leven voor de direct betrokkenen. Epidemiologisch onderzoek heeft een duidelijk verband aangetoond tussen lage rugpijn en de mechanische belasting op wervelkolom. Gedurende het ouder worden treden biochemische- en structurele veranderingen op in de tussenwervelschijf. Deze veranderingen hangen vaak samen met veroudering. Een theoretisch model is nodig om de complexe chemische en mechanische interactie te bestuderen. Het model dient rekening te houden met de chemische en structurele samenstelling van het weefsel.

Het model zoals beschreven in dit proefschrift is gebaseerd op de mengseltheorie: het weefsel is opgebouwd uit drie componenten: vaste stof, vloeistof en kleine ionen. Op basis van kinematische relaties, algemene behoudswetten en de tweede hoofdwet van de thermodynamica worden constitutieve verbanden afgeleid. Deze relaties zijn afhankelijk van zowel de lokale deformatie als de lokale chemische samenstelling van het weefsel. Het model is gebaseerd op: (1) eindige vervormingen van de vaste stof, (2) relatieve vloeistofstroming veroorzaakt door gradienten van de chemische potentiaal van de vloeistof en de ionen, (3) niet-ideale Domnan osmose en electroneutraliteit, (4) convectiediffusie van de kleine ionen. Het model beschrijft de evenwichtsresultaten van een confined compression experiment in het fysiologische gebied voldoende nauwkeurig. Het model laat ook zien dat de schijnbare afhankelijkheid van de elastische constanten veroorzaakt wordt door Donnan osmose.

De vergelijkingen die het chemo-mechanische gedrag van tussenwervelschijf weefsel beschrijven, zijn drie gekoppelde partiële differentiaal vergelijkingen. Om deze op te lossen voor een willekeurige geometrie en willekeurige randwoorwaarden is gebruik gemaakt wan de eindige elementen methode. Een total Lagrange (Newton-Raphson iteratie schema, derde orde Houbolt tijdintegratie) formulering is geimplementeerd in het commerciele pakket DIANA (DIANA Analysis B. $V .$, Deitu). Voor de numerneke studies worden iso-parametrische serendipity elementen gebruikt. Twee dimensionale wlakke rek, axiaal-symmetrische en drie dimensionale elementen zijn ontwikkeld. De interpolatie functies zijn gelijk voor de verplaatsing, druk en jonconcentratie. De orde van de interpolatie functies varieert van lineair tot cubisch.

Voor de experimentele validatie van het model is gebruik gemaakt van de een dimensionale confined compression test. De proefstukjes zijn verkregen uit de anulus fibrosus van herderachtige honden. De oriëntatie van de proefstukjes is evenwijidig of loodrechtten opzichte van de wervelkolon. Het belastingsprotocol bestaat uit een combinatie van chemische- en mechanische belasting. De experimentele data kan gefit worden op het model. Het zwel gedrag wordt veroorzaakt door Donnan osmose. De experimentele resultaten geven geen aanleiding tot het invoeren van een zout afhankelijke elasticiteitsmodulus of een chemische uitzettingscoëfficiënt. De elasticiteitsmodulus and de permeabiliteit zijn vervormingsafhankelijk. Zowel de elasticiteitsmodulus als de permeabiliteit verschillen significant voor de axiale en radiale proefstukjes. 
De gevolgen van het wee of drie fasen modelleren is bestudeerd aan de hand van axiale compressie van een bewegingssegment zonder facet gewrichten. De berekende vervormingenen en vloeistof stroming als funktie van de tijd zijn in overeenstemming met gerapporteerde experimentele resultaten. Donnan osmose beinvloed de spanningen in de vezels en de grondmatrix, en de vloeistof stroming door het bewegingssegment.

Het gepresenteerde model voor het chemo-mechanisch gedrag van tussenwervelschijf weefsel is in staat om de respons van het weefsel op een chemische en/of mechanische belasting te beschrijven. 